\title{
DIE SPRACHE DER NORTHUMBRISCHEN INTERLINEARVERSION ZUM JOHANNES-EVANGELIUM.
}

\section{Vorwort.}

Im jahre 1894 erschien Albert S. Cook's "Glossary of the Old Northumbrian Gospels" (Lindisfarne Gospels or Durham Book), Halle, Niemeyer, in dessen vorwort der autor auch eine northumbrische sprachlehre für die nächste zeit ankündigte. Etwas früher schon hatte Miss Mary Elizabeth Lea ( $=$ Mrs. Wright) in der Anglia XVI, 62-206 eine abhandlung über die sprache der north. interlinearversion zum Markusevangelium veröffentlicht. Seitdem sind sechs jahre verflossen, ohne dass die Cook'sche grammatik, die doch bereits "ready for the printer" war, erschienen wäre, auch ist inzwischen von anderer seite an die drei übrigen north. evangelien noch nicht hand angelegt worden; so darf ich es vielleicht im folgenden unternehmen, der interlinearversion zum Johannesevangelium eine sprachliche untersuchung $\mathrm{zu}$ widmen.

$\mathrm{Zu}$ grunde gelegt habe ich den text von W. W. Skeat: The Gospel according to St. Natthew etc. in Anglo-Saxon and Northumbrian Versions, Cambridge 1878-87. Eine beschreibung der hs. findet sich in Skeat's "Preface" zum Markusevangelium, sowie bei Cook: Biblical Quotations in Old English Prose Writers etc., London 1898.

Beim Johannesevangelium sind zwei schreiber thätig gewesen; die zweite hand beginnt nach Skeat's bericht in der "Preface to St. John's Gospel" mit $\hbar$ ou geniomae kap. V, v. 10 in roter tinte, weicht aber von dem sprachgehrauch der ersten in keinerlei weise ab. Die verwendung von $g i$ für $g e$ ist keine eigentümlichkeit von ihr, wie schon Skeat gegenüber Waring 
betont; sondern $g i$ erscheint überwiegend erst vom ende des kap. XX ab bis am schluss auf p. 188. Offenbar in verbindung mit diesem umstand steht eine andere erscheinung, deren Skeat nicht gedenkt, nämlich dass $i$ von da ab willkürlich für alle möglichen vokale in unbetonter silbe eintritt, auch wo es etymologisch nicht die geringste berechtigung hat.

Ich habe folgende belege notiert: ueri 3 . sg. opt. XIX, 28; giseni n. sg. masc. XX, 20; gisîi 1. sg. XX, 25; noesi adv. XXI, 5; neri 3.sg. opt. XXI,7; oðri n. pl. masc. XXI,8; sêgni acc. sg. XXI, 8; 才iu segni n. sg. XXI, 11; (elni geändert zu elno XXI, 8 rd.); gloêdi n.pl. XXI, 9; hlingindi gen.pl.part.XXI,12; dagi d.sg.XXI, 14; petri d. sg. XXI, 15; $u$ бi (=be?) XXI, 17; cueði 1. sg. ind. XXI, 18; fylgendi acc. sg. masc. XXI, 20; gircesti 3. sg. ind. praet. XXI, 20; gcuuni inf. XXI, 22; syndrigi acc. pl. neutr. XXI, 25; magi 3. sg. opt. praes. XXI, 25; aurittenni inf. XXI, 25; gihamadi 3. sg. praet. 188, 7; cuðberhti d. sg. 188, 8. 10; sancti d. sg. 188, 10. Mit einem alten etymologischen $i$ haben wir es hier also keinesfalls $\mathrm{zu}$ thun; vielmehr erinnert diese verwendung des buchstabens in unbetonter silbe lebhaft an die schreibart nordenglischer texte des 13. und 14. jahrh. Die aussprache mag ungefähr wie die heutige oberbairische in unbetonter silbe gewesen sein (vgl. haibi, gšerti, koan ${ }^{i}$, glerti). Eine erklärung für das plötzliche auftauchen des $i$ bei einem schreiber, der dicht daneben übrigens auch die sonst üblichen endungsvokale richtig schreibt, lässt sich nicht leicht geben, vielleicht darf man an einen wechsel der vorlage denken, wenn auch der biedere Aldred versichert, sich allein um die übersetzung des evangeliums verdient gemacht $\mathrm{zu}$ haben. In dieser neuen vorlage mochte das archaische $i$ bewahrt sein; von unserm schreiber, der das nicht mehr verstand, wurde es dann ohne regel auch für andere endungsvokale eingesetzt. Die Skeat'sche erklärung für gi neben ge "Preface" p. X scheint mir unwahrscheinlich.

Was die darstellung des stoffes betrifft, so habe ich mich streng nach der einteilung in der Sievers'schen grammatik gerichtet und bin namentlich überall vom westgermanischen lautstande ausgegangen, der ja doch für das anglische ebenso gut wie für das westsächsische die vorstufe bildet. Für die verschiedenen lauterscheinungen alle belege, bezw. die gesammtzahl der einzelnen anzuführen, lag nicht in meiner absicht, da ich ja hier überall auf Cook's, erschöpfendes 
"Glossary" verweisen durfte. Auch die rein graphischen varianten, namentlich die ganz bedeutungslose scheidung von $a$ und ae S. $\S 6$ anm. 1, sowie das nebeneinander von $u$ und $w$ bezw. $u u, u w, w u$ habe ich nur in gewissen fällen besonders berücksichtigt; genaue angabe dar schreibart findet sich ebenfalls bei Cook.

\title{
Teil I. Lautlehre.
}

\section{Erster abschnitt: Vokalismus.}

\section{A) Die vokale der stammsilben.}

\author{
Kapitel I. Kurze vokale.
}

§ 1. $-a$ -

1) In ursprünglich geschlossener silbe erscheint westgerm. $a$ in unserm denkmal fast durchweg als $a$ wie im ws. Die belege sind zahlreich sowol vor einfacher oder doch nur vom schreiber verdoppelter, als auch vor mehrfacher konsonanz. Beispiele für die erstere gruppe bieten zunächst die 1. 3. sg. des praeteritums der starken verba nach klasse IV und $V$ (s. auch unter stammbildung der starken verben): bcedd XIX, 38; gebod IV, 47; gebar XIX, 5; gebrac XIII, 26; cuød VI, 36. 65; XIII, 33 etc. (nebst zusammensetzungen 127) (daneben cued s. unten und cwed mit graphischen varianten s. unter $w$-einfluss); ongoet V, 6 ; XII, 9; ongatt VI, 15; XVI, 19; XVII, 25 (2); (daneben ongeat I, 10; IV, 1 ; s. unter wirkung vorausgehender palatale); gelag V, 3; sat Prf. 6, 13; VIII, 2; IX, 8; gesatt IV, 6 (6) (daneben set Mt. Prf. 21, 2; sętt Pt. Prf. 19, 8); sprcec Prf. 4, 4; XV, 11; XVI, 6; XVI, 25; spracc X, 6; XVII, 1; sprôe XVI, 33 (einmal sprêc = locutus est $\mathrm{s}$. unter stammbildung der starken verben); ferner das praeteritum der wurzel wes: woes Prf. 1, 5; 2, 2. 3; III, 23 etc. (203); uass IX, 25 (daneben was Mt. XXI, 42; Mk. XVI, 2; L. XXII, 59; węs Mt. Prf. 8,$7 ; 20,7$; II, 3; vgl. S. § 49 anm. 1); nces VII, 39 (3); noess $\mathrm{XI}, 15$; ebenso das praeteritopraesens maeg V, 19. 30; sowie der imperativ des starken verbums nach VI fara: far VII, 3; doch vgl. auch S. $\S 49$ anm. 2; und Bülbring, Anglia, Beiblatt IX, 89-91.

Weiter sind hier zu nennen of S. $\S 51 ; \S 130$ in afista II, 17 S. § 43 anm. 4; at, aed Prf. 4, 3; III, 23; VIII, 38; XI, 29 
(10); aet-, aed- Prf. 7,13; V, 20; X, 32; XXI, 13 (37); (wegen $-t$ : $-d$ s. unter dentalen); back XVIII, 6 ; baeccling VI, 66; bagcgling XX, 14; bcerlice XVI, 29; -doeg V, 9. 10; IX, 14; goers VI, 10 (mit metathese aus groes); fatt XIX, 29; lehtfat V, 35; gloed III, 29; glcednise Prf. 7, 16; III, 29 (5); hucs XIX, 24; hucet, huced Prf. 3, 13: 7, 5. 11; II, 25 etc. (54); бoes Prf. 1, 3. 12; 2,6; I, 27 etc. (56); бot III, 19; V,2; VI, 21 (gewöhnlich wiedergegeben durch $\bar{f}(182)$ ).

Neben $a$ ist einmal ge- oder wol richtiger verschrieben ece in becedd XIX, 12.

Vor mehrfacher konsonanz findet sich a bei: after- Prf. 1, 1; IV, 46. 54; VIII,2; (efter) XII, 22; foest III,33; V,30; XVII, 25 etc. (7); foestlice III, 18; VII, 41; IX, 22 (8); gebraegd XVIII, 10 ; gefragn IV, 52 (daneben gefraign XVIII, 7. 19; s. unter north. diphthongen und gefregn Mk. V,9); in den partizipien praet. von habba und sacga, die ohne westgerm. mittelvokal gebildet sind, S. § 415, 1: hafde, nafde etc. Prf. 4,2; IV, 18; IX, 41; XV, 22. 24 etc.; sagde Prf. 6, 4; I, 15; III, 28; XI, 46; XIV, 2 etc.; gescegd Prf. 3, 3; 8, 1, bei hrceglum XIX, 40; hucøre VI, 6; VII, 13; XIII, 7; (daneben hueðre Prf. 4, 2; IV, 27; IX, 41; XIII, 13 (7)); huoedre Prf. 2,4; X, 6; XV,22; XVII, 25 (7). (Auch für das verhältnis dieser beiden formen gilt wohl, was S. $\S 342$ anm. $1 \mathrm{zu}$ hwoeðer : hweðer, hwoeðer bemerkt, dass nämlich die letztern beiden auf altes $\ddot{e}$ hinweisen); in dem

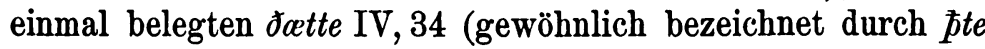

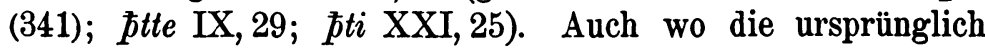
geschlossene silbe durch sekundäre lautentwickelung im ags. offen wird, oder wo sich ein sprossvokal einstellt, bleibt dies $a$ : hragl XIX, 5 s. oben; uastm XII, 24; XV, 2. 5. 8; hier schwand das $h$ (vgl. *wahsan), ehe es brechung und weiterhin palatalumlaut hervorrufen konnte, s. S. §165 anm. 3; foeðem I, 18; (monn)-magen XVIII, 3; uaestem Prf. 2,6. 6, 15; IV, 36; XV, 2. 4. 16.

$e$ kommt abgesehen von den schon erwähnten fällen nur vor in hehstald,-nise Prf. 1,2.3.4.5 (6) - von Cook sonderbarerweise als héhstald angesetzt - in der schreibung scheint allerdings eine anlehnung an héh $=$ ws. hêah vorzuliegen. cued II, 21. 22; III, 12; VIII, 24 etc. (mit zusammensetzungen 70, s. oben) verdankt sein $e$ dem regellosen durcheinander, das in der schreibung dieses wortes herrscht, wie sich andererseits 
auch oft genug ae im praes. findet, näheres s. unter stammbildung der starken verben, vgl. S. § 391 anm. 10 .

$a$ weist auf $a h(a c)$ und seine zusammensetzung mit ne: Prf. 3, 5. 15; III, 15. 16; IV, 12. 29. 35 etc. (128). Hier verhinderte wohl die satzunbetontheit die erhöhung zu $a$, s. S. $\S 49$ anm. 1 .

$c$ findet sich in folgenden lehnworten: moessa VII, 2; scegnade VIII, 48; für vulgärlat. $\dot{e}$ bezw. $\breve{\imath} \mathrm{s}$. Pogatscher p. 2-7; und in daccille Prf. 4,12; paccille V, 35; daccillum XVIII, 3, das sicherlich eine beiform zu facula-facele ist. Wegen des anlauts s. Kluge, Et. wb. die artikel über "dämmerung" und "finster".

cecclesice 188,1 hat wohl als lat. wort zu gelten, dessen schreibung dem ae. angenähert ist.

2) In ursprünglich offener silbe erscheint westgerm. $a$, teils als $a$, teils als $a$.

$a$ steht, wenn die folgesilbe einen der gutturalen vokale $a, o, u$ enthält:

Beispiele aus der flexion des verbums sind: aron XVII, 22; aro XIII, 11; arun XXI, 16 rd.; aru VIII, 41; gefara I, 43; gegladade VIII, 56; hafo IV, 32; X, 18 etc. (9); hafu IV, 17; V, 36; gelaðad Prf. 1, 9; magon VIII, 22; Prf. 7, 10 etc. (4); mago V, 44; VIII, 6 ; XIV, 5 etc. (8) (daneben einmal mogon XIII, $36 \mathrm{~s}$. unten); geondsuarade III, 10; V, 19; geondsuarede I, 50; IV, 13 (7) (daneben auch formen mit wurzelvokal $a, e a, o$ S. $\S 416$ anm. $13 \mathrm{c}$, s. unter stammbildung der schwachen verba II); getaled VI, 10; S. § 414 anm. 4. Anderweitige belege: dagas Prf. 6, 11; IV, 40.43 etc.; dagum Prf. 3,13; 8,6; II, 12.19 etc.; dagana XX, 1. 19; fadores I, 18; II, 16 etc.; fadrum Prf. 2, 2 (-dr bewirkt keinen silbenschluss); fatto II, 6; lehtfatum XVIII, 3; hradur XX, 4; nacod XXI, 7; monnslaga Prf. 5, 16; sacleas $\mathrm{XV}, 25$ (nur scheinbar geschlossene silbe, da ursprünglich sacu bestand, ebenso tal VI, 10, das aber eigentlich für tale steht und zur nächsten gruppe gehört); sadal XII, 14 (= asald?); foresaga 187, 14; stafum V, 47.

Bemerkung. $a$ erscheint als $a$ in einer reihe von wörtern, in denen auf den ursprünglich gutturalen vokal der mittelsilbe ein $i$ folgt oder folgte, vgl. S. $\$ 50$ anm. 2: hlcetmesta VI, 39; VII, 37; hlatmestae VI, 54; latmeste $\mathrm{XVI}, 23$ etc. $(10)(<$ *latumist). 
(In unserem denkmal zeigen auch die infinitive und partizipia praes. der starken vb. VI $a$ : farende VII, 35 (2); XVIII, 1; saccendum Prf. 6,14; gesaeccanne Prf. 8, 4; aber lâdanne IV, 7 (über -anne s. S. § 363 anm.2. Grundform *annjai, *onnjai, doch haben hier wohl weitgehende ausgleichungen die ursprünglichen verhältnisse getrübt, vgl. S. § 371 anm. 8).

a kommt ausserdem vor in frero XIV, 3; XVI, 7.17 (und hafo Mt. VII, 9; L. XII, 17); sago III, 3. 12; XIII, 21; XV, 15; auch hier dürfte analogischer einfluss vorliegen (näheres s. unter stammbildung der verben); endlich in burguarum I, 19, wo sich wohl formen von -waru und -wearas, -waras, -woras (s. unter einflüssen des $w$ ) miteinander vermischt haben.)

Vor ursprünglichem $\mathrm{d}$.h. nicht aus $a, o, u$ geschwächtem $e$ steht in der regel $a$ : daeges XI, 9; dage II, 23; V, 9 (11); dagi XXI, 14 (2); darg (dat. sg.) Prf. 4, 19; 5,4 etc. (7) s. flexion der $o$-stämme 2. 3. sg. ind. praes. von habba und sacga: haefes, høefed, haefis, høefid III, 15; IV, 11. 18; VI, 68; VIII, 48 etc. (31); - sages, sageठ, saegeð Prf. 3, 6; 8,2; IV, 25; XVI, 13. 15 etc. (9); < *sagaip, *habais S. § 415; gladdde XVI, 20; hræðе XI, 29; hrêઇe XIII, 27; raeðe XX,15; s. auch S. § 315 anm. 2; hucðer III, 4; VII, 52 (daneben viel häufiger hueðer IV, 12. 29. 33 etc. (11); huoeder VII, 41. 47.48 etc. (8). Von diesem nebeneinander gilt dasselbe wie von hucedre, huedre, huoeðre s. oben 1); S. § 342 anm. 1); uceter Prf. 3, 10; IV, 14 etc.; ucetter XIX, 34; ucettre I, 26 (-tr ist silbenanlaut); gedafenlic IV, 4 (gedoefenlic Prf. 4,1 - hier oe sicherlich verschrieben für $a e$, doch vgl. mit anderer ablautsstufe gedéfe Beow. 1227 u. ö.). In diese gruppe gehören von haus aus auch die formen des häufig belegten verbums gedafna, wenn auch durch die synkope frühzeitig silbenschluss eintrat. Es kommen vor: gedofneð, gedaefneð Prf. 6, 10; III, 7; gedœfnað, gedaefnað III, 30; IX, 4; X, 16; XIX, 7; gedcefnade, gedaefnade Prf. 1, 9; XX, 9; gedoefnad Prf. 2, 4; IV, 20. 24; gedoefned XIII, 14. Ich bin absichtlich so ausführlich in der aufzählung der belege gewesen, um zu zeigen, dass das einmal vorkommende gedeafnad III, 14 nur ein schreibfehler für gedaefnad sein kann; von $u$-umlaut kann keine rede sein, denn einmal wird in unserem denkmal $a$ davon überhaupt nicht betroffen, andereits sind die bedingungen dazu gar nicht vorhanden, da anzusetzen ist: *gedafenad. 
Schwanken zwischeu $x$ und $a$ herrscht vielfach in den formen der flexionen unter dem einfluss des systemzwanges, $s$. S. $\S 50,2$. So bei faeder: nom. sg. faeder V, 20. 21. 22; XVI, 15 etc. (58, darunter 11 mal faeder); fader Prf. 4,9; 6, 7 (7); acc. sg. foeder Prf. 4,12; 5, 17 etc. (27, darunter 7 mal faeder); fader Prf. 7, 9; XIV, 7 (3); feminina der $\bar{a}$-klasse: ondsucere I, 22; ondsuare XIX, 9 (ondsuere Mt. II, 12); optative praes. der starken verba VI: faere XVI, 7 (2); faere XI, 7; hlada IV, 11 (über die endung - $a$ vgl. unter flexion der verba); (partizipium praet. ofsloegen Mk. IX, 3; L. IX, 22).

$a$ steht in folgenden lehnworten: calic XVIII, 11, s. Pogatscher p. 69 ; sacerdas $\mathrm{I}, 19$; sacerda XII, 10 ; mit $\bar{a}$ nach Pog. $\S 186$; S. $\S 12$. § 50 anm. 5 ; aseldes Prf. 6, 13; asaldes XII, 15; assald XII, 14; apostol XIII, 16.

3) Westgerm. $a$ wird $o$ (nicht $\varphi$ ) in den proklitischen praepositionen: of Prf. 7, 13; I, 15. 40; V, 31. 32. 36. 37. 46 etc. (223); on I, 43; IV, 44. 45; VI, 15 etc. (143); ôn VII, 2; XVIII, 20; 188, 7; onfoe V, 34.41 etc.; onginned Prf. 1, 1 etc.; ebenso ठone, ठonne Prf. 1, 3; 3,2; I, 15 etc.; Prf. 5, 14; II, 10 etc.; huonne VI, 25; instr. huon XIII, 28; Jon Prf. 7, 15; ebenso gehört hierher wohl auch huodhuoegu V, 14; huothuoegu XIII, 29; huodhuoegu VII, 4.

Ueber ursprüngliches $a$ in schlussgliedern von kompositis s. dort.

$a$ vor $l$-verbindungen $\mathbf{s}$. bei der darstellung der brechungsverhältnisse.

Westgerm. $a$ wird in seiner geltung eingeschränkt:

1) durch den übergang in $Q$ vor nasalen s. dort;

2) durch die brechung $\mathrm{zu}$ ea vor $r$-verbindungen s. dort auch $a$ vor $h$ - und $l$-verbindungen;

3) durch diphthongierung $\mathrm{zu} e a(e c e)$ nach vorausgehendem palatal s. dort;

4) durch kontraktion zu $\bar{a}, e \bar{a}$ s. dort;

5) Durch $i$-umlaut zu $e, a$ s. dort.

$$
\S 2 . \quad-e \text {-. }
$$

Westgerm. $\ddot{e}$ hat in unserem denkmal ungefähr denselben spielraum wie im ws.; nur sind hier die wirkungen des $u$ - und o/a-umlauts ausgedehnter, und ausserdem die einflüsse eines 
vorausgehenden $w \mathrm{zu}$ berücksichtigen; indes findet eine beeinträchtigung durch vorausgehenden palatal in der regel nicht statt, vgl. die bemerkungen von Lind. und $\mathrm{L}$.

Beispiele flir $\ddot{e}$ aus den flexionen der starken verba nach III, IV, V; berað XXI, 10; berende XIX, 5 ; únberende XV, 2 rd.; сиеðо I, 51; gecueðas II, 5; gecuedne Prf. 1, 13; bidelfa XIX, 40; eted X, 10; gefregn imp. praes. (= ws. i) XVIII, 21; gefregne XVI, 5 (zu gefregna vgl. Kluge, P. G. 933); gefe XVI, 2; forgefes, forgefen $\mathrm{XX}, 23$; ongetto $\mathrm{X}, 14$; ongette $\mathrm{X}, 38$; ongetted VII, 17; ongeten Prf. 8, 4; helpend V, 7; forrepene Prf. 5, 8; S. § 391 anm. 1; gesegn (part.praes.) I, 50; spreco IV, 26; sprecces XVI, 13; spreceð Prf. 5,6 ; sprecas III, 11 ; sprecað III, 31 ; spreccende I, 37; gestele X, 10; gesuelta IV, 47; gesueltae XVIII, 14 .

Bemerkung. Ueber die formen mit $u$ - und $o / a$-umlaut sowie mit $w$-einfluss s. dort; die 1. sg. sowie der plural ind. von sprecan lassen sich auch als formen mit $u$ - bez. o/ $a$ - umlaut fassen, die durch den folgenden palatal vereinfacht wurden, vgl. S. § 164 anm. 2.

Sonstige belege für $\ddot{e}$ sind: gebed Prf. 2,2; efne Prf. 1,7; gefo I, 17; godspell Prf. 1, 4; godspellere Prf. 1,2; (derne) -legerscip VIII, 4; mec, meh Prf. 7, 5; 8, 10; I, 15 etc.; mêc XV, 25; ઇec, ðeh I, 48,50; XVII, 5 etc.; gemet II, 6 rd.; stefn Prf. 3,5; ðegnas Prf. 7, 3; ðegn I, 43; ðegnum Prf. 3, 8 (daneben zahlreiche formen mit ei s. unter north. diphthongen); des (= hic) Prf. 1, 13; I, 7. 15. 33 etc.; uel II, 6 rd.; uêl XVIII, 23; uêg XIV, 5; auez IV, 50 (daneben -oe s. unter $w$-einfluss); ueras VI, 10 (daneben $a, a(e a)$ s. unter $w$-einfluss und $u$-, o'a-umlaut); spere XIX, 34.

a findet sich geschrieben in spracende VI, 63 ; gespracend $\mathrm{X}, 6$; spreccend XVIII, 21; sucefnes XI, 13; uces imp. XIX, 3 (sehr oft in den praesensformen von cueða s. auch unter $w$-einfluss).

Ueber hueðer, hueðre vgl. unter hucðer, huc®re.

Germ. $\ddot{e}$ erscheint als $o$ in

$e$ findet sich in folgenden lehnworten: cesto XIII, 29 (ws. ciest; vglat. cesta); plett X, 1 (plecta $\mathrm{B}$.-T.); sestre II, 6 rd. (< sextarius, *sehst-, wo $h$ schwand, ehe es brechung und weiterhin palatalumlaut hervorrufen konnte, vgl. waestm, auch deutsch sester Kluge, Et. wb.); tempel II, 19. 20; tempul Prf. 3,13; temple Prf. 3, 13 etc.

Westgerm. $\ddot{e}$ wird eingeschränkt: 
1) durch den übergang in $i$ vor nasal s. dort;

2) durch die brechung vor $r$-verbindungen; s. dort auch $e$ vor $h$ und $l$-verbindungen;

3) Durch den $u$-umlaut zu eo (ea) s. dort;

4) durch einflüsse eines vorausgehenden $v$ ev. in verbindung mit $u$-umlaut.

\section{§3. $-i-$.}

Westgerm. $i=$ idg. $i$ findet sich in der dritten stammform der starken verba nach I: fordrifeno XII, 42; begrippene Prf. 5,8; stige IV, 47 (ofstigon VI, 16 etc. s. unter palatalumlaut); besuicen VII, 47; unslitten XIX, 23; besmitten XVIII, 28; uuriten VI, 31 ; auritten II, 17 etc.; ausserdem in folgenden beispielen: cuic IV, 11; Eadfrið 188,1; billfrið 188, 4 (über bill (sword) s. K. L.); fisc XXI, 9.13; fisces VI, 9 etc.; geflitt IX, 16; hidder IV, 15; hidir VI, 25; him Prf. 1, 6; 3,3 etc.; hine Prf. 3, 4; 4, 2; 6, 8 etc.; his Prf. 1, 3. 14; hîs XVI, 17; hir Prf. 8,3; IV, 7 etc.; hit Prf. 4, 16 etc.; hlingindi XXI, 12; lifed IV, 53; VI, 51; lifde IV, 51; lifiende VI, 51.57 etc.; hwisprendo Prf. 4,20; geniđrad Prf. 5, 9; geniðrað VIII, 10; huidder VIII, 14; huidir III,8; XII,35 etc.; toslitnise Prf. 6,12; tui(spreccendo) Prf. 4, 20 (steht wohl zu twā im ablautsverhältnis); scipp VI, 17; Jider II, 2; đidder VI, 21; wisneð XV, 6, s. S. § 382 anm. 3; wiste Prf. 1, 14 etc.; uittnesse I, 7; megulit $\mathrm{V}, 37$; urit $\mathrm{XX}, 9$ etc.

Bemerkung. $i$ in hripes IV, 35; hrippe IV, 35 gehört vielleicht hierher, s. Sievers, Beitr. X,506; S. $\S 382$ anm. 3 ; vore. ist $i$ in denen auf -scipe S. $§ 98$ anm.; argscipe Prf. 5, 8; dernelegerscip VIII, 4 etc. Ueber giues IV, 9 etc. s. L. $\S 51$ note; S. $\$ 416$ anm. 14 b.

$$
i=\text { germ. } i \text { aus idg. } \ddot{e} \text {. }
$$

a) Vor nasal + konsonant: getimbred II,20; in den praesensformen der starken verba nach III, a: blimpe V, 14; dringad Prf. 5, 7; drincad II, 10 rd.; drinco XVIII, 11; onginneð Prf. 1, 1 etc.; gesingað XIII, 38; bringað XXI, 10; s. S. § 407 anm. 15; im verbum substantivum sint Prf. 5,8; I, 2 etc.; sindon Prf. 67 ; VIII, 33 etc.;

in anderen wörtern: blind IX, 2. 13. 18 etc.; blind IX, 24; ofblindade XII, 40; fingre VIII, 6; inting XVIII, 38; XIX, 6; intîng XIX, 4; oing XIII, 29; winde VI, 18; uinter X, 22; geuintrad XXI, 18; winnes subst. Prf. 2,6. 
b) Vor ursprünglich folgendem $i, j$ : in den praesensformen der starken verba biddan (idg. aber wz. *bhidh, vgl. griech. $\pi \varepsilon(\vartheta \omega)$, licgan, sittan: gebiddas Prf. 7, 11 etc., sittendo II, 14 etc.; licgende V,6; ferner bei soðcuido X, 6; Jignen XVIII, 17; is Prf. 1, 2 etc. (idg. ${ }^{*}$ esti); micelo V, 3 (vgl. aber auch $\mathrm{K}$. L. unter much und mickle); middum I, 26 etc.; milsa 188, 11; earlipprica XVIII, 10 (über den vokal des suffixes s. K., NSt. $\S 68 \mathrm{~b})$; sib Prf. 7,8; unsibsumnise Prf. 6,1; sig(beg) XIX, 2; gesigfaestnad Prf. 8,12 ; sighðana Prf. 1, 11; insiht Prf. 1, 1; scillinga XII, 5; smiride IX, 11 ; smirinise Prf. 6,11 ; S. $§ \S 159$ anm. 1; 408 anm.1; spillo VI, 39; spilded XII, 25; sticadun XIX, 37 (s. auch palataluml.); feortig II, 20; fiftih Prf. 8,9 etc., s. Nor. $§ § 71,3 ; 92$ anm. 1; 93; uil III, 8 etc.; uillo I, 13; losuistes XVII, 12.

Bemerkung. $y$ für $i$ findet sich durchgängig bei symle, symble VI, 34; VII, 6 etc. (liegt hier vielleicht eine labialisierung dnuch $m$ vor?); einmal in wynn IV, 38 (aber oben winnes Prf. 2,6).

Für ws. willað findet sich stets uallad; uallas XII, 21; XXI, 3 etc., S. § 428, das ich nicht anders zu erklären weiss, als durch annahme einer $i$-losen bildung mit der ablautsstufe $o$; vgl. die ahd. formen wellemês etc. mit umlants-e $\mathrm{Br}$. $\S 385$ und anm. 3, die also auch o-stufe haben.

Lehnworte: biscop XI, 49; biscobe XVIII, 24 etc.; cirica $\mathrm{X}, 22$; discas II, 15;

$i>i$ bei ausfall eines nasals vor tonlosem spiranten fif Prf. 4, 14; suide Prf. 7, 11.

$i$ erfährt einschränkung:

1) durch die brechung $\mathrm{zu}$ io vor $r$-verbindungen etc. s. d.;

2) Durch den $u$ - und o;a-umlaut zu io (ia) ev. in verbindung mit vorausgehendem $w \mathrm{zu} u \mathrm{~s}$. dort;

3) durch kontraktion s. dort.

\section{§ 4. - - o-}

Westgerm. o bleibt unverändert:

in den part. praet. der starken verben nach II, III, IV: tobrocene XIII, 26; eft-boren III, 5 ; gecoren Prf. 1,2; gedolfene Prf. 2, 1; gefrognen Prf. 5, 12; agolden Prf. 1, 13; gotteno Prf. 6,11 ; tostrogden XI, 52; geworden Prf. 1,6; touorpen XI, 52;

in anderen worten: bibodo Prf. 7, 6; bebohton II, 16; unboht $\mathrm{XV}, 25$; bolla XIX, 29; cofa XI, 38 (S., Beitr. X, 497), gecostade 
DIE SPRACHE DER NORTHUMBR. INTERLINEARVERSION. 11

VI, 6; ofercostung XVI, 33; fole Prf. 6,13; folce Prf. 6, 10; for, fore, befora, forma - meist bezeichnet durch f'e, f'ma etc.; forohtandum Prf. 4, 16; gode Prf. 1, 2; godspellere Prf. 1, 2; golde 188, 5; hlott XIX, 24; locc Prf. 6, 2; losas Prf. 4, 17; losuistes XVII, 12; morgen XVIII, 28; oft I, 12; smolt XX, 19; rocgetede 187, 14; trog XIII, 5; dornum XIX, 2; dohter XII, 15; wordes Prf. 1, 6; uôrd X, 21.

Bemerkung. In einigen wörtern erscheint, zumal in der nachbarschaft von labialen, eine answeichung des $o$ nach $u$ : full $\mathrm{I}, 14$; ungeleaffulle Prf. 4, 3; lufu Prf. 7, 10; lufandum Prf. 7, 6; ufa Prf. 3, 7; uulf X, 12.

Bemerkung. Für ws. uolde, uoldon erscheint ualde Prf. 6, 9; ualdon VI, 11; vgl. oben żu wallað; o in ठorfendum XII, 5; ðorfä XIII, 10 ist wohl vom praet. dorfte bezogen.

Lehnworte: apostol XIII, 16; copp XIX, 29; corona XIX,2; costum Prf. 7.10, portas V,2; zur gruppe full etc. gehört wohl culfrce I, 32; culufro II, 14; culfero II, 16, falls die deutung aus colubra Kluge P. G. I, 337 richtig ist. Ueber eine neue etymologie von culfre $\mathrm{s}$. jetzt Holthausen I. F. X, 112, der eine durch suffix -bh- erweiterte, auch in slavischen sprachen vertretene wurzel $g l-$ zu grunde legt.

Westgerm. $o$ wird eingeschränkt:

1) durch den übergang in $u$ vor nasal s. dort;

2) durch $i$-umlaut zu $e$ s. dort.

$\S 5 .-u-$.

Westgerm. $u$ weisen auf das praet. pl. der starken verba nach II sowie praet. pl. und part. praet. der starken verba nach III (s. dort): getugon VI, 52; gebundeno XI, 44; indrungno II, 10; gefrugnon I, 19; gesungen Prf. 2, 2; gewundun XIX, 2; uundun XIX, 2; wunnon IV,38; geurnun XX,4; gwurnun IV,51; auurpon VIII, 59.

Einmal ist der vokal des plurals in den singular eingedrungen: gisprunt 187,14; andererseits findet sich einmal auorpon IX, 35; in anlehnung an die formen des praes. und part. praet.

Sonstige belege: burg XI,18; burug V,2 etc.; burna XVIII,1 (mit metathese s. unter $r$ S. $\S 179,1$ ); cunnon I, 26; cunnedon VIII, 6; heofoncund VI, 31 rd.; utacund X, 5; duru Prf. 6, 2; Jus XI, 48; hund, hûnd, hundrað Prf. 8, 9; VI, 7; XVIII, 12; lust 
Prf. 1,$3 ;$ sum $\mathrm{V}, 5$; suundur $\mathrm{XX}, 7$; suno Prf. 3,3 ; sunnedoeg Prf. 4, 9; under I, 50; uundra Prf. 8, 1; uuldor I, 14 (wo u für zu erwartendes $o$ sich wohl durch die annahme eines ursprünglichen es/os-stammes erklärt; got. $u$-stamm: wulpus).

$u$ wird bei ausfall eines nasals vor tonlosem reibelaut $\mathrm{zu} \bar{u}$ : ûs Prf. 2, 5; ws XIII, 29; uusc- (bearn) XIII, 33; cuðlice Prf. 3, 11; сибе I, 31; тиље XIX, 29; wuðuotto VIII, 3 (die schreibung $w u$ steht hier wie oben in gwurnun IV, 51 für uu und bezeichnet die länge des vokals, s. L. § 10,3).

$u$ findet sich auch in dem ganz unverständlichen dusidi XXI, 14 rd., bei dem wir es vermutlich mit der gedankenlosen zusammenschreibung von abkürzungen zu thun haben, vielleicht $\delta u s$ in $\delta y$ ? s. auch Zupitza im glossar zum "Uebungsbuch".

Lehnworte: luh VI, 1 ; purbple XIX, 5; zweifelhafter herkunft ist fuluande Prf. 3, 7 etc., wenn es nicht doch zu lat. fullo $\mathrm{zu}$ ziehen ist.

$u$ wird eingeschränkt:

1) durch $i$-umlaut zu y s. dort;

2) vereinzelt durch übergang in iu nach palatalen.

Kapitel II. Lange vokale.

Die bezeichnung der vokallänge durch accente ' (') oder doppelschreibung ist in unserem denkmal gar nicht selten, bei einzelnen wörtern sogar die regel, s. L. § 28. Ich verzichte darauf, eine liste der so als längen kenntlich gemachten vokale vorzuführen und werde die handschriftlichen längebezeichnungen bei den einzelnen lauten notieren. Ueber die längung kurzer vokale und ihre bezeichnung $s$. unter schwankungen der quantität.

$$
\S 6 . \quad-\bar{a}-.
$$

Westgerm. $\bar{a}$ (= germ. $\bar{a}$, ws. $\bar{x}$ ) ist im allgemeinen durch $\bar{e}$ vertreten, daneben finden sich einige wenige beispiele für $\bar{x}$, s. L. $\S 44$.

Dies $\bar{e}$ tritt auf in der 2. sg. und im plural indic., sowie im ganzen optativ des praeteritums st. vb. IV, V: bedon IV, 31; gebêdon XIX, 31; gebreco XIX, 31; gebrêcon XIX, 32; gecuede VII, 9 ; cuedon II, 18 (daneben formen mit oe s. unter $w$-einfluss und mit $a$ S. unten); geeton VI, 13; geêton VI, 23; ongetton VI, 69 ; ongêto VIII, 55; gesegon Prf. 8,8; I, 14; gesege VI, 5; 
DIE SPRACHE DER NORTHUMBR. INTERLINEARVERSION. 13

VIII, 57; gesêgon XXI, 9; aseton VIII, 3 ; seton XX, 12; missprecon VI, 41 (formen mit $a$ s. unten); were Prf. 1, 7; uere I, 48; ueron Prf. 1, 14; 3,2 etc. (81) (daneben formen mit oe $\mathrm{s}$. unter $w$-einfluss und $a$ s. unten); nêre XVIII, 30 ; nere XXI, 23; nerce XII, 42; in den formen des praes. und part. praet. einiger reduplizierender verba: ondredeð XIV, 27; forleta Prf. 7, 8; forlettes XI, 48; lêttes XVI, 2; eftforletno Prf. 7, 17 etc.; redendum Prf. 1,8; slepe Prf. 6,8; slepeð XI,11;

in anderen wörtern: bêre Prf. 4,8; beer V, 9; geres XI, 49; Jer Prf. 1, 9; 3, 11 (44) (formen mit a s. unten); herum XII, 3; hêrum XI, 2; huer I, 39; VIII, 10.19 etc. (8) (formen mit oe s. unter $w$-einfluss; mit $a$ s. unten); huér I, 38; huêr XX, 2; lesua $\mathrm{X}, 9$; meg III, 29; mêg XI, 11; nêdrae III, 14; sprec VIII, 43; sprêc IV, 42 ; XI, 47; settere $\mathrm{X}, 1$; setteras $\mathrm{X}, 8$ (von Cook fälschlich als sęttere angesetzt); umlautsbedingungen sind vorhanden bei fêtels IV, 28; megð IV, 54; VII, 1. 41 etc.; meghð I, 43 (über formen mit -ei s. unter north. diphthongen, mit $a$ s. unten); dêd Prf. 1,6; dedo Prf. 8,1; sêd VII, 42; sêel 188,11; teles XII, 48. $\bar{e}$ findet sich ausserdem in dêdon VI, 23 (sonst formen mit $y$, s. unter $i$-umlaut zu $u$ ); geêt II, 17, vgl. Kluge P.G. § 171, p. 436; S. § 391 anm. 3 ; erendureca IX, 7; erendureco XX, 12 rd. s. Kluge, Beitr. VI, 385. 386; Bugge, ib. XXIV, 431 ff.; dagegen aber S. $\S 100$ anm. 4 .

Bemerkung. Schwanken zwischen $\bar{e}$ und $a$ zeigt sich bei hucer IV, 11 s. oben; $\partial œ r$ IV, 46 ; XIX, 42; cucelon VIII, 57; gesprace IV, 27; uæere $\mathrm{XX}, 15$; uaron XXI, 8; n仑̂̀e XI, 32; naerun XXI, 8, bei denen anlehnung an den vokal des singulars stattgefunden haben mag, vgl. auch cueð unter $a$ in geschlossener silbe und S. § 391 anm. 10.

Niemals $\bar{e}$ weist auf: westgerm. ${ }^{*}$ su $\bar{a}$, das zwischen suce Prf. 2,$2 ; 7,5$ etc. (44) und sua XIII,15. 33; XIV,31 etc. (7) schwankt und jedenfalls verkürzt ist.

Auf ursprünglich germ. $\hat{e}$ (idg. $\bar{e}$ ) deuten wohl auch die $\bar{a}$ der verba bláwan (cnáwan), sáwan in den formen des praes. und part. praet.: forblauene VI, 18 ; saued IV, 36 ; saues IV, 37; vgl. Braune, Got. gr. § 22; Kluge P.G. § 114, p.407; § 165, p.432; dagegen S. § $57,2 \mathrm{a}, \S 62 \mathrm{anm}$.

Nicht ganz durchsichtigen ursprungs ist $\bar{a}$ im verbum gān (doch s. Kluge P. G. $§ 166$, p. 433): gâa XVI, 5; gaces XIII, 36; gaað III, 8 etc. (Die häufig vorkommende schreibung $a a$ ist sonach wahrscheinlich nicht als $\bar{a}$, sondern als wurzelvokal 
plus endungsvokal zu deuten, vgl. auch die formen von dōa, onfōa s. S. § 430 anm. 1).

Westgerm. $\bar{a}$ wird vor nasalen zu $\bar{o}$ s. dort auch germ. $\tilde{a}$ aus $a(n) h$; über $\bar{a}$ in der umgebung von palatalen s. dort.

$$
\S 7 . \quad-\bar{e} \text {. }
$$

Ueber germ.-westgerm. $\bar{e}$ vgl. Kluge P. G. $\S 126$, p. 411; $\bar{e}$ erscheint in her I, 41; V, 20; XI, 21; hêr XI, 32; über den vokal in redon XIX, 20; onfengon I, 12. 16; ahengon XIX, 18; s. S. § 394 anm. $2 ; \S 395$ anm. 3.

- Auf kontraktion beruht das $\bar{e}$ in êce Prf. 4, 3; III, 15. 16.

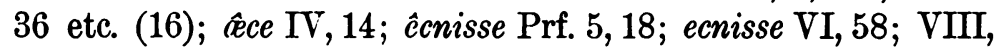
35. 51; acnisse IV, 14; s. Kluge, NSt. § 212.

Lehnworte: feber ( $a d l$ IV, 52, s. Pog. § 118; cregisc XIX, 20; cregesc XX, 24; grecisc XXI, 2; s. Pog. $§ 141$.

\section{$\S 8 . \quad-\bar{\imath}$.}

Westgerm. $\bar{\imath}$ hat seine stelle in den praesensformen der st. verba nach I: biddendra $\mathrm{V}, 3$; todrifed X, 12; gegripa XII, 35 (über formen mit io s. unter $u$-, o/a-umlaut; s. S. § $382 \mathrm{anm} .3$ ); gehrîne XX, 17; gihrînade 188,4; s. S. § 382 anm. 4; aris Prf. 4, 8; arisad Prf. 4, 11; arisa XX, 9; astigo XX, 17; gescined I, 5; scinende V, 35; besuicað VII, 12; toslîte (ue) XIX, 24; auritteঠ VIII, 6; stigende I, 32;

dann in zahlreichen anderen wörtern: gecîdon VI, 52 ; Jin Prf. 4, 7 etc.; Jriim II, 19; Jrim Prf. 3,13; Jrîim 188, 7 etc.; hrîpes, hrippe IV, 35 (s. auch unter $\tau$ ); hrining Prf. 7, 3; huile Prf. 7, 14; huito IV, 35; gelic Prf. 5, 1; gelíc IX, 9; lichoma Prf. 1,6; lif Prf. 4, 3; lîf XIV, 6; lîn XIII, 4; linninum XIX, 40; min II, 4; mîn VI, 38; sidum Prf. 8, 7; sîdu XIX, 34; sinum XXI, 14; sciu XIX, 31; s. S. § 277 anm. 2; stið VI, 60; geuidloed XVIII, 28; tîd Prf. 4, 11; uifes Prf. 1,3; û̂f XVI, 21; uitga Prf. 3,6; genitgade Prf. 2, 1; uisum Prf. 7, 10.

Lehnwörter: ficbeam I, 48; crist Prf. 3,2.5 etc.; rîc Prf. 3,15; XVIII, 36; aus dem an. stammt dîr ancilla XVIII, 17.

\section{§ $9 . \quad-\bar{o}$.}

Westgerm. $\bar{o}$ begegnet im indikativ und optativ praet. der st. verba nach VI: drog XXI,11; drogun XXI, 8; geðuoh Prf. 7,1 ; ahof VIII, 7; ahofon X, 31; onsoce Prf. 8,11; onsôc I, 20; 
gestod I, 26; stód VIII, 44; stodun XI, 56; slog XVIII, 10; gewox Prf. 4, 1; ausserdem bei blod VI, 55; blôd XIX, 34; boc Prf. 1,11; broder Prf. 3,9; broðer I, 40; doa Prf. 4, 15; doende Prf. 4, 9 etc.; feoðordoger Prf. 6,8; dom Prf. 5,11; dôme XVI, 8; ठro uende Prf. 7, 12; drouunges Prf. 7, 19; god II, 10; fotum XI, 32; feoðorfota IV, 12; fostrað VI, 31; flouad VII, 38; behofad $\mathrm{XI}, 50$; eftlocade I, 36; moder Prf. 1, 4; rûmmôda XIV, 26; rummóda XV,26; more IV, 20; môtêrn XVIII, 28; pol V, 2; rouing $\mathrm{XXI}, 8$; rode Prf. 1,4 ; rôde XIX, 19; unrotnisses Prf. 7,15; gesoht Prf. 4, 17; scoes I, 27; to Prf. 1, 4; tô XI, 32; suot XII, 3; stoue Prf. 2,2 ; stôu XIV, 3 .

In lehnworten erscheint $\bar{o}$ als vertreter des nord. au, S. $\S 26$ anm.; brydlopum Prf. 1,3. 8; s. Kluge, P.G. 932; ôra 188,10; ora 188,$9 ;$ s. S. $\S 26$ anm.

Auslautendes $w \bar{o}$ erscheint als $\bar{u}$ in $h u$ XVIII, 22; huu III, 4; und tuu XVIII, 12 rd., vgl. S. §60 anm.

$\bar{o}$ erfährt $i$-umlaut $\mathrm{zu} \bar{\alpha}$ s. dort.

$$
\S 10 .-\bar{u}-
$$

Westgerm. $\bar{u}$ erscheint unverändert in bruc IV, 31 ; gebrucced VI, 50; bruceð VI, 57 etc.; vgl. S. § 385; Kluge P. G. §161, p. 430; (lond-) bucend Prf. 7, 9; buta IX, 22; bûta XV, 6; neheburas IX, 8; adune Prf. 2, 1; Jusendo VI, 10; hus Prf. 4, 7; rummôd Prf. 7,7 ; rûmmôda XIV, 26 ; run Prf. 3,14 ; rúnum Prf. 4, 4; snude III, 3, 7; trugude II, 24; lehtun XVIII, 1; tuune XXI, 2; ûruh XIX, 23; ut IX, 35; it VI, 37; îte XVIII, 16; uuteode VIII, 59; uutearde XVIII, 22; ymbhuungun VII, 22.

$\bar{u}$ erleidet $i$-umlaut zu $\bar{y}$ s. dort.

\section{Kapitel III. Diphthonge.}

\section{§ $11 .-a i$ -}

Westgerm. ai erscheint wie im ws. als $\bar{a}$ : in der 1.3. sg. ind. praet. der st. verba nach I: abad VIII, 9; fordraf II, 15; gehran Prf. 8, 7; arâs II, 22; gestag Prf. 2, 1; astāg VI, 17; awrat Prf. 1,11; aurât VIII, 8; anderweitige belege sind: aganes Prf. 3,13 rd.; âgno X, 3; (feber-) adol IV,52; an Prf. 3,8; ân Prf. 1,2 ; armorgen XVIII, 28; ârmorgen XX, 1 ; ârlice XXI, 4 ; aare IV, 44; arwyrðe Prf. 4,10; bân XIX, 36; бa Prf. 1, 10 etc.; ठâ XVIII, 18; đas I, 28; đâs XVII, 25; facon I, 47; frasendum Prf. 
Prf. 3,4; gaste Prf. 3, 7; gâst XIX, 30; gaaste III, 8; gâast XIV, 26; halge Prf. 3, 7; unhalra V, 3; hâl V, 9; hamas XIV, 2; hâme XI, 20; gehatne Prf. 1,14; gihamadi 188, 7; hlafum Prf. 4,14; lafe VI, 13; lâf XIII, 26; lam IX, 6; inlâde 188, 9; lar Prf. 2, 6; lâr XVIII, 14; laruu Prf. 2, 6; gelahton XVIII, 12; s. S. § 407 anm. 10-12; gemana Prf. 1, 3; mara Prf. 7, 2; maasto $\mathrm{XV}, 13$; racing Prf. 8, 11 (daneben racing Prf. 7, 3); rapum II, 15 ; rahte XIII, 26; tahte Prf. 8, 12; s. S. § 407 anm. 11 ; sauel Prf. 6, 3; spadle IX, 6; stan VIII, 7; tacon Prf. 1, 7; tacnas Prf. 3, 13 rd.; tân XIX, 24; uat IV, 2J; wât VII, 27; uast III, 8; nastu III, 8; naast XIII, 7; nât XII, 35.

Vereinzelt erscheint $\bar{o}$ statt $\bar{a}$ : oht XVIII, 9; noht I, 3; VIII, 54; s. S. § 62 anm.

Ueber $\bar{a}$ in forblauene VI, 18 ; saued IV, 36 s. unter $\bar{a}$.

Lehnworte: caser XIX,12. 15; coesares XIX,12, s. Pog. §199. Altengl. $\bar{a}$ erfährt $i$-umlaut zu $\bar{a}$ s. dort.

\section{$\S 12 .-a u-$}

Dem westgerm. au entspricht hier wie im ws. ein éa, neben dem nur vereinzelt éo erscheint. Dies éa weisen auf: die 1.3. sg. praet. der st. verba nach II: bebead Prf. 1,4; geceas VI, 70; gecêas XIII, 18 (über geceason XV, 16 s. unter stammbildung der st. verba (II)); ofgat II, 15 mit offenbarem schreibfehler vgl. L. $\S 56,1$; über aspeaft IX, 6 s. S. $\S 384$ anm. 5; weiter findet sich éa bei ficbeam I, 48; bread XIII, 27; brêad VI, 23; deaðes Prf. 2, 3; deado Prf. 4, 10 (daneben daed $\mathrm{XIX}, 7$; dâed XIX, 33 verschrieben s. ofgoet); eac Prf. 1, 11; III, 23; XI, 5.48 (sonst $a c, e c, e c e c$ etc. s. unter palatalumlaut); Eadfrið 188, 1; eadgo Prf. 8, 8; eaðe VIII, 19; carlipprica XVIII, 10; eorlippric XVIII,26; eastro Prf. 3,$12 ; 4,14 ; \mathrm{II}, 13$ etc.; eostro II, 23; XIX, 14; eostres XII, 1 etc.; heafut XIII, 9 ; hêafut XX, 7; heafde XIX, 30; XX, 12; hafde XIX, 2 (schreibfehler vgl. doed etc.); heannissum VIII, 23 (vgl. wastem S. § 165 anm. 3); ungeleaffulle Prf. 4, 3; leas VIIl, 44. 55; leasunges Prf. 5, 16; felleread XIX, 5; screadunga VI, 12; stream I, 28; esneteam IX,22 rd.; Jreatum Prf. 4, 17; Jrêat XII, 9 ; in der stellung vor $w$ : aedeaunise Prf. 8, 1; 2,1; aedeauas XIV, 22; aedeaude XXI, 1; aedeaued Prf. 4,4 etc.; ymbsceaude I, 42; insceaunge Prf. 8,7, s. auch unter $-w$ - und unter stammbildung schw. verba).

Lehnworte: ceapadon IV,8. 
Ae. e $a$ wird eingeschränkt:

1) durch $i$-umlaut $\mathrm{zu} \bar{e} \mathrm{~s}$. dort;

2) durch palatalumlaut $\mathrm{zu} \bar{e} \mathrm{~s}$. dort.

$$
\S 13 .-e u-\text {. }
$$

Für westgerm. eu, dem ws. regelmässig êo entspricht, gilt als normalform in unserem denkmal $e a$, neben dem die schreibung éo nur vereinzelt sich findet, vgl. S. § 150 anm. 1 .

Beispiele bietet das praesens der st. verba nach II: bebeadas Prf. 7,19; bebeado XV,14; einmal bebeodo XV,17; beadande Prf. 8, 11; hleatte XIX, 24 (oferleorað Prf. 4, 10; oferleora XIII, 1, s. $§ 18 \mathrm{~d})$.

Sonstige belege: éo in breost XIII, 25 (daneben brest XXI, 20; Mt. Prf. 6, 14 vielleicht mit palatalumlaut s. dort); éa: ðeaf Prf. 6, 3; X, 1 etc.; ðeade IV, 43.

In den praeterita der reduplizierenden verba gehealde Prf. 1, 5 ; beheald I, 36 etc.; feoll VI, 31 rd.; IX, 38; XVIII, 4; feollon XII, 13; XVIII, 6 ; feall XI, 32; oncneaun XII, 16; ondreard XIX, 8; ondreardon VI, 19 ; forleort IV, 3 etc.; sowie im praeteritum eade IV, 47. 50; X, 40 etc. (14); eadon IV, 30; eadun XVIII, 6 ; geeadon VI, 66; eode IV, 43; VII, 1 etc. (9); eodun $\mathrm{XX}, 10$; XXI, 3 ist der diphthong reduplikationsprodukt $\mathrm{s}$. Kaluza $\S \S 159$. 192,2, vgl. auch unter stammbildung reduplizierender verba.

$\overline{e a}(\bar{e} o)$ aus westgerm. -iun findet sich in seado XII, 6; XIII, 29 (got. siujan).

1) Schon westgerm. wurde eu vor folgendem $i, j \mathrm{zu} i u$ und dies wird in unserem denkmal weiter zu $\bar{\imath} o$ umgelautet (gegenüber ws. $\bar{\imath} e$ ), s. unter $i$-umlaut.

2) $\bar{\imath} o$ sowie $\bar{e} o$ werden durch palatalumlaut $\mathrm{zu} \bar{\imath}$ bez. $\bar{e}$ s. dort.

$\S 14$. Speziell northumbrische diphthonge.

In der stellung: palataler (kurzer oder langer) vokal plus palatale konsonanz entwickelt das northumbrische gern ein epenthetisches $i$, das mit dem vokal einen diphthongen bildet; und zwar erscheint dieser meist als $e i$. Westgerm. $\ddot{e}$ liegt zu grunde bei ðeign I, 45; ðeignas Prf. 1, 14 etc. (7) (daneben ðegn s. unter $\ddot{e})$; forleigere VIII, 41; auch im lehnwort reigluord IV,46 (daneben regluord IV, 49); auch für ursprünglich $i$ kommt auf- 
fallenderweise der diphthong vor: Jritðeih Prf. 4, 8 (Cook liest Jriteih); teigða I, 39; $\bar{e}$ (= ws. $\bar{\alpha})$ liegt zu grunde in meigठ Prf. $1,11$ (s. auch unter $\bar{a}) ; \bar{e}(=$ ws. $\bar{e}$ ) bei onfeing XIII, 4; onfeingon I, 11 (s. auch unter $\tilde{e}) ; \bar{e}$ (< urengl. $\bar{e} a+i$ ) bei deigle XIX, 38; deiglice Prf. 5, 4; deigilnisi 187, 14; heig VI, 10; ceigunge Prf. 6, 8; und viele formen des verbums ceigan: ceiged Prf. 4,12; ceiges $\mathrm{XI}, 28$; ceigde $\mathrm{XI}, 28$; ceigdon $\mathrm{IX}, 24$; ceig IV, 16; geceiged Prf. 3,9 (im ganzen nur zwei formen mit $\bar{e}$; einmal geciged I, 42; falls hier nicht ein schreibfehler vorliegt, ist wohl anzunehmen, dass ei infolge der sehr geschlossenen qualität der ersten komponente stark $\mathrm{zu} i$ hinüberneigte, dann liesse sich vielleicht auch gihrîno 188, 4 hierherstellen $\left(<{ }^{*}\right.$ geręine $<{ }^{*}$ geręigne < gereggne, s. auch unter $-g$-); mittelglieder wären etwa formen wie geceid L. I, 35; teiða Mt. Prf. 3, 18); auf $\bar{e}<\bar{e} a$ durch palatalumlaut geht zurück ei in gebrêicon VI, 31 mit analogischer übertragung des singularvokals in den plural, $s$. unter stammbildung der st. verba II.

ai findet sich nur in gefraignad IX, 21; gefraignas IX, 23; gefraigne XVI, 19; gefraigna XVI, 30; gefraignes XVIII, 21; tostraigdes X, 12; auch im praeteritum gefraign XVIII, 7; es scheint überhaupt die schreibung ai auf *ai zurückzuweisen, indem $a$ aus dem singular praet. den ursprünglichen praesensvokal $\ddot{e}$ in diesem verbum fast ganz verdrängt hat, vgl. S. $\S 155,3$.

Kapitel IV. Beeinflussung betonter vokale durch nachbarlaute.

$\S 15$. Einfluss der nasale.

1) Westgerm. $a$ erscheint vor nasalen fast durchaus als 0 .

Beispiele: vor $m$ und $m+$ cons. from Prf. 1, 2 etc.; lichoma Prf. 1,6; noma Prf. 7,6; lomb Prf. 3,6; somnung Prf. 6,10; gesomnadum Prf. 2, 5; uommbe III, 4;

vor $n$ und $n+$ cons.: brondum XVIII, 3 ; conn VIII, 55 ; Jone Prf. 1,3; 3, 2 etc. (daneben Jene); ठon Prf. 1, 5 etc. (instr.); huon IV, 11 etc. (instr.); Jonne II, 10 etc.; huonne VI, 25; vgl. auch S. § 65 anm. 2; huona I, 48; hona XIII, 38; ealond Prf. 1,11; lond(bucend) Prf. 7,9; monigo Prf. 4,4; monigfald Prf. 6, 15; monigfaldlice Prf. 7, 3 (daneben menig); ond-: ondsuare XIX, 9; geondete Prf. 3, 6; ondetend I, 20 etc.; hond XI, 44; 
hônd XIII, 9; hónd XX, 20; stondas III, 29; stôndende XVIII,16; uiðstonden Prf. 5, 7; mon II, 10; aldormonn II, 9;

vor gutturalem nasal: Joncunge VI, 11 (geonga s. unter diphthongierung durch palatale); longunga Prf. 7, 18; gemong XIX, 39; gemônges XIX, 39; đuong I, 27; ondfong 188, 10.

$a$ findet sich bei and XXI, 19 (sonst stets ausgedrückt durch das zeichen 7), sowie ausnahmslos im sing. praet. ind. der st. verba nach III auf nasal + konsonanz: gearn Prf. 6, 12 mit metathese, S. § 386 anm. 3; doch Bülbring, Anglia, Beibl. IX, 97; dranc IV, 12; ongann Prf. 1,6; gesang XVIII, 27; gesuanng XIX, 1 (zu swęngan; das aber wohl mit st. vb. swingan kontaminiert wurde.

$a$ findet sich auch in der 1 . sg. praes. ind. des vb. subst. -am Prf. 1,13 etc., das wohl direkt auf ein $(a m>){ }^{*}$ ommi $>$ *ormi zurückgeht, während die ws. formen der wurzel es mit anlehnung an die wurzel bheu (1. sg. Ps. biom) gebildet zu sein scheinen; L. § 427; doch s. auch S. § 43 anm. 2.

Lehnworte: vor einfachem nasal bleibt $a$ erhalten in canone $\mathrm{XIX}, 30 \mathrm{rd}$., und ebenfalls vor nasal + konsonant in ambeht XXI, 23; ambihta XX, 30; ambehtum XXI, 14 (sonst mit umlaut embeht, so regelmässig im kompositum embehtmonn); angelica Prf. 8, 3; angla I, 51 (sonst mit umlaut engel); $a$ vor nasal + kons. erscheint als $o$ in comp (uearod) XVIII, 12; oncroe 188, 4; (hefid)- ponna XIX,17. In dieser stellung einmal $u$ bei cumpadi XIII, 36 3. pl. opt. praet.

2) Diese $\rho$ werden gelängt $\mathrm{zu} \bar{o}$ bei ausfall des nasals vor stimmlosem reibelaut: oðrum Prf. 3,10; ôdro XX, 25; soðlice Prf. 2, 3; sôðXVI, 5 .

3) Schon vor der zeit der einzeldialekte war germ. $a$ vor $n h$ nach ausfall des nasals zu nasaliertem $\bar{a}$ geworden. Dieses $\bar{a}$ erscheint ebenfalls stets als $\bar{o}$ : gebrohte Prf. 8,5; geðohton XI, 53; ahoh XIX, 6; onfoas Prf. 3, 3 etc.; s. auch unter kontraktion.

4) Westgerm. $\bar{a}$ (= germ. $\bar{x}$ ) wird vor nasalen zu $\bar{o}$ : cuomon Prf. 8,5; cuome (2. sg.) VI, 25; cuome (opt.) XV, 22 etc.; fornomon I, 5; genome (2. sg.) XX, 15; genome (opt.) XIX, 38 etc.; $\bar{o}$ für zu erwartendes $\varphi$ ist auch der singularvokal des praet. ind. dieser beiden verben: cuom I, 31 etc.; genom V, 9 etc. (S. $\S 390$ anm. 2); moneðo IV, 35; sona Prf. 3, 10; vielleicht gehört hierher auch der vokal von huôn XIV, 2. 
Dieses $\bar{o}$ ist dem $i$-umlaut unterworfen s. dort.

5) Westgerm. $\ddot{e}$ wird vor altem $m \mathrm{zu} i$ : nim Prf. 4, 8; nimað XX, 23 etc. Dieses $i$ erleidet $u$-, o/a-umlaut $s$. dort.

Vor $m+$ kons. ist $e \mathrm{zu} i$ geworden im lehnwort gimmum 188,5 .

6) Westgerm. $o$ vor (einfachem, S. $\S 70$ anm.) nasal wird zu $u$ : cumas XXI, 3; cummende I, 29 (daneben weitaus häufiger formen mit $y$ s. unter stammbildung der st. verba); brydguma II, 9; fruma Prf. 1, 13; benumene Prf. 1,7; Juner XII, 29; geunia Prf. 4,3; wunande Prf. 7, 7 .

Lehnworte: pûnda XIX, 39.

Der $i$-umlaut dieses $u$ ist $y$ s. dort.

\section{$\S 16$. Die brechungen.}

1) Vor $r+$ konsonanz.

$a$ in dieser stellung wird zu ea gebrochen: bearn Prf. 3, 9; uusc- bearn XIII, 33; bearm I, 18 (barme L. XVI, 22. 23; barm L. VI, 38); earde IV, 44; earnunga 188, 12; hearnunge Prf. 7, 10 ; ofcearf XVIII, 10; middangeardes Prf. 3,7 ; uingearde XV, 4; gearua VII, 6 ; foregearuung XIX, 14; lindis-fearnensis 188, 1 ; lecueard XX,15; durenêard XVIII, 17; forueard- (mercunga) Prf. 3, 1; toueard Prf. 8, 12; ondueardnese Prf. 4,6; geonduearde Prf. 5, 13; geondueardon II, 18; untearde (= wearde) XVIII, 22; heartlice XII, 32. Nach $w$ herrscht grosse mannigfaltigkeit in der lautgebung, neben formen mit $e a$ finden sich häufiger solche mit $a$ oder $a$, s. unter $u$-einfluss.

Ueber die formen mit $o \mathrm{~s}$. unter minderbetonten silben.

Befremdlich ist das ausbleiben der brechung bei argscipe Prf. 5, 8 (arga L. Prf. 8, 18; $\arg$ Mt. XII, 39); vgl. L. § 11, 1 ; Lind. § $9 \mathrm{I}, 1$; dazu stellt sich ingebarg II, 9; ferner arm XII, 38; nedðarf (Luk. Prf. 2, 8; XIV,18; XXII, 7; XXIV,4); XVI, 7. 30 ; verschrieben nedfarð XIII, 29 (ðarf Mt. XIV, 16; むarfe L. XXI, 3 etc., s. Cook); daneben stehen formen mit $a$ : nedðarf Prf. 3, 12; ðcerf II, 25; むaerfe IX, 8 (ðaerfē Mt. V, 3 rd., únðorfe Mt. II, 6); (ðorf' XII, 6; ठorfe L. Prf. 9, 3 haben ihren vokal wohl aus dem praet. ठorfte (L. part. ðorfende S. § 422 anm. 3) bezogen); nur einmal ea: đearf Mt. VI, 8 . Vielleicht infolge von schwachtonigkeit bleibt ohne brechung die 2. sg. praes. ind. des vb. subst. arst, arð I, 19. 21 etc., s. L. § 13, 3. 
Ungebrochener vokal findet sich auch in dem etymologisch schwierigen wcerlde IX, 1 (biwarlas L. XI, 42; biwarlde L. X, 31; ymbwarlde L. VII, 9. 44; X, 23; ymbwaelde L. IX, 55). Da dies wort an fast allen zitierten stellen die bedeutung "convertere" hat, liegt es nahe, an eine intensivbildung zu hweorfan zu denken, vgl. ahd. warbalôn; wegen $a$ s. Bülbring, Engl. St. XXVII, 86; über den abfall des anlautenden $h$ s. S. § 217 anm. 1 ; freilich lassen sich keine parallelen für den ausfall des $f(=z)$ in dieser stellung finden.

Die brechung unterbleibt regelrecht, wenn die $r$-verbindung erst durch metathese entstanden ist, s. S. $\S 79$ anm. 2: gaers VI, 10 (L. XII, 38; gers Mt. VI, 30; XIII, 26; XIV, 19; groesum Mk. IV, 32); ebenso in den lehnworten carcern III, 24; carr I, 42.

Die brechung von $a$ vor $r+$ konsonant, abgesehen von der stellung nach $w$ und in minderbetonter silbe muss demnach als die regel betrachtet werden: die wenigen ausnahmen besonders in der stellung vor $r g$ und $r f(r[f] l)$, vgl. auch ws. haerfest $\mathrm{S}$. § $79 \mathrm{anm} .2$, bedürfen noch einer näheren erklärung (s. Kaluza p. 102).

Die brechung von $\ddot{e}$ vor $r+$ konsonant erscheint als $e o$ In eorðlico III, 12; eorðu VIII, 8; XXI, 8; corઇo III, 31; XII, 32 etc.; eorðe VI, 21; VIII, 6 etc. (eo: Mt. Mk. L. J. zs. 95; formen mit ea s. unten); heorta XIII, 2; XIV, 27 etc. (einmal herte L. VIII, 15; die formen mit ea s. unten); reordana Prf. 7, 10 (über den vokal s. Lind. p. 13 anm.).

als $e a$ in cearfanne Prf. 5,3 ; gecearfa VIII, 37. 40; ymbcearfas VII, 22 etc. (niemals eo); earðo XII, 24 (earðes L. XII, 56 ; earðu Mk. IV, 31; earðo Mt. Prf. 18, 14 etc.; ea: Mt. Mk. L. J. zs. 8; eo s. oben); mearda IV, 36 (einmal merdo L. Prf. 9, 14); niemals $e o$;

als io in briorde II, 7 (daneben brearde Mk. XIII, 27); giorne $\mathrm{XX}, 11$ (vielleicht in anlehnung an das schw. verbum giorna); sonst georne Mt. Prf. 15, 4; II, 4.7 etc.; geliornade VI, 45; s. auch S. §416 anm.12; riordade IV,27; s. oben reordana; hriordad XXI, 12.

Die brechung des $e$ in dieser stellung ist also bis auf zwei fälle, die vielleicht nur schreibfehler sind (s. Zeuner p. $24 \mathrm{anm}$.), durchgeführt; und zwar stehen $e o$ und $e a$ promiscue nebeneinander; die formen mit io erinnern an kentische schreibertradition, 
$i$ vor $r+$ konsonant, das fast nur vor früher folgendem $i, j$ vorkommend einem vorgerm, $e$ entspricht und im ws. über io weiter $\mathrm{zu}$ ie umgelautet wird, erscheint als io ohne von dem ursprünglich folgenden umlautsvokal beeinträchtigt zu werden; s. S. $§ 79,2$.

Die beispiele sind selten: hiorde Prf. 6,3; X, 2.11.12.14. 16 etc.; giornde IX, 8 (giornade Mk. X, 46; giornede Mk. I, 40; giorndon L. V); iorsiges VII, 23.

Trotz der metathese, die aber auch Bülbring, Anglia, Beibl. IX, 97 für älter hält als die brechung im angl., ist $i$ gebrochen in den formen von iorna: iornendum Prf. 8, 3 (ymbiornas Mt. Prf. 8, 3; iorned L. XXII, 10 etc.).

Eine eigentümliche form ist bearnende $\mathrm{V}, 35$, das wohl in die analogie der übrigen st. vb. III auf $r+$ kons. übergetreten ist und für ursprüngliches io: eo/ea eingesetzt hat; s. S. §79 anm. $2 ; \S 386$ anm. 2 ;

geliornade VI, 45 s.oben; die formen von smiriga s. unter germ. $i<e$ vor $i, j$.

\section{2) Vor $l$ + konsonanz.}

$a$ in dieser stellung bleibt ungebrochen: ald III, 4; aldum Prf. 1, 9; aldormonn II, 9; III, 1 etc.; aldormen II, 8 etc.; aldro IV, 20; VI,31. 49 etc.; aldrum VII,22; VIII, 9; aldorleasa XIV, 18; all Prf. 6, 13; IX, 34 etc.; alle VI, 37; allo XVIII, 20; allra Prf. 7, 1; allum Prf. 3, 7 etc.; cald XVIII, 18; tuufald Prf. 1, 3; anfalde, drifalde 187, 11; tuifallice Prf. 7, 10; gefâlden XX, 7; haldo VIII, 55; halda XV,10; gehalden Prf. 2, 6; ahaldan V, 4 etc.; halfe X,1; half XXI, 6; halle XVIII, 28; halscôde XI, 44; hascode XX, 7; haltra V, 3; gesalde Prf. 3,13 ; 7, 1; gesaldes XVII, 2. 6 (2). 7. 8. 9. 12. 24; gesald I, 17 (asâld Mt. XXVIII, 18; $a$ im (umlautslos gebildeten S. § 407) praet. ind. und opt.; sowie part. praet. bei Mt. Mk. L. J. zs. 215; formen mit ea s. unten); saltes IV, 14 (glossiert salientis, das der schreiber offenbar für eine adjektivbildung zu sal hielt); hehstald Prf. 1,2. 5. 13; hehstaltnisse Prf. 1, 3; Ediluald 188, 2. 3.

Demgegenüber kommt $e a$ nur vor in den formen des verbums sealla, wo es im infinitiv auf o, $a$-umlaut zurückzuführen ist, s. dort, und von da aus vereinzelt in das praeteritum ind. und opt. eingeschleppt wurde; von letzteren formen sind belegt: sealdes XVII, 4. 11; sealdon XIX, 3 (sealde Mt. XXV, 15; 
XXVI, 27; Mk. XIV, 22 etc. bei Mt. Mk. L. J. zs. 10); gefealla XII, 24 ist, obwohl es das praesentische cadens glossiert, jedenfalls als opt. praet. zu fassen und entspricht ws. gefeolle.

Lehnworte: palma Prf. 6,12; palmana XII, 13; palmung $\mathrm{XV}, 2$; über asald vgl. L. $\S 12,1$.

Brechung von $\ddot{e}$ vor $l+$ kons. findet nur statt bei seolf Prf. 3,5.11; seolfe V, 30; seolfum I, 22; seolfa IV, 42; XI, 55; XVI, 13 etc.; vgl. hierzu die erklärung bei Dieter II, p. 773.

3) Vor $h$.

In dieser stellung sind alle brechungen durch palatalumlaut vereinfacht ("geebnet" Bülbring, Anglia, Beibl. X, 3) worden; spuren lassen sich vielleicht aber noch erkennen, wo $h$ zwischen vokal und stimmhaftem konsonant am frühesten schwindend (Bülbring, Anglia, Beibl. IX, 107) wohl brechung, aber keine "ebnung" mehr veranlasste. So: eorodmonna XVIII, 12 rd.; $(\ddot{e}>e o$ ); ferner angl. $\bar{e}$ (ws. $\bar{e})>\bar{e}$ in geneolecde Prf. 4, 14; II, 13; geneolecdon XII, 21; geneolicde Prf. 3, 12 etc. (98); (ca nur bei Mt. $5 \mathrm{mal}$; $e$ ebd. $3 \mathrm{mal}$ ). Bülbring a. a. 0.107 erblickt freilich in diesen formen kontraktionsprodukte; doch s. S. § 165 anm. 3.

Kaum in betracht kommt eahtu Prf. 4, 8 (eahteða Mt. Prf. 9,8); dafür sonst durchgängig $a$.

Als lehnwort gehört hierher getrahtad I, 38. 42; getrachtad I, 41; getractad IX, 7; dazu wohl auch verschrieben tretiað XIV, 26, wo die brechung unterblieb.

\section{Die umlaute.}

\section{$\S 17$. Der $i$-umlaut. \\ I. Umlaut von $a$.}

Westg. $a$ vor ursprünglich einfachem konsonant (= urengl. $a)+i$ erscheint in der regel als $e$ : bero VI, 9 ; bed (beer) V, 8; betre IV, 52; elno XXI, 8; elnung II, 17; gedd X, 6; ahefes Prf. 5, 13; ahefeð VIII,28; ahefen XII,32 (s. S. § 378 anm.1); hebbendum XI, 41 etc.; hefignise XVI, 21; here XII, 18. 29; nett XXI, 6 ; metto IV, 8; selenises Prf. 7, 19; sello, selo IV, 14; selles $\mathrm{XXI}, 20$; sel IV, 7; sellende VI, 64 etc. (formen mit ea und $i \mathrm{~s}$. unter $u-, o ! a-$ uml. und unter wirkung vorangehender palatale); settis XIII, 38; settanne $\mathrm{X}, 18$; sette $\mathrm{XV}, 13$; im praet. wurde der umlaut bei diesem vb. sekundär eingeführt (S. § 407); gesett Prf. 
1, 7; gesetton VI, 10 etc.; frumsetnung XVII, 24; bisuêđdun XIX, 40; aðenes XXI, 18; auecco II, 19; aueco VI, 40; auecced II, 20; aueccee VI, 44 etc. (e: Mt. Mk. L. J. zs. 9, sonst $c e(o c)$, s. unten);

a findet sich in onsacces Prf. 3, 4; XIII, 38; aucecced V, 21 eftwaccende Prf. 4, 19 etc. (bei Mt. Mk. L. J. zs. $a$ 30; $e$ s. oben, daneben formen mit oe s. unter $w$-einfluss); smcell XVIII, 22 ; hondsmoellas XIX, 3; vgl. S. § 89 anm. 1.

Westgerm. $a$ vor konsonantgruppen (= urengl. $a)+i$ erscheint als $a$ in faestas VI, 63; faestern Prf. 5, 8; noeht III, 2; VII, 50 etc.; als $e$ in eft Prf. 8,6; XI, 38. 46 etc.; esne Prf. 7, 2; VIII, 35; êsnces XVIII, 18; êsnum XVIII, 26 etc. (nur einmal cesne-monn X, 13; môtêrn, sprêcêrn XVIII, 28 (vgl. S. § 43,3; $\S 89$ anm. 4; Bülbring, Anglia, Beibl. IX, 97); restdaeg XX, 19 (daneben rostendra XXI, 12; $e$, $\nsim$ 12); s. S. $\S 89$ anm. 2; über gihrino 188,4 (<* ${ }^{*}$ geręgno) s. $\S 14$; vgl. auch L. $\S 23$ note).

Lehnworte: aecced XIX, 29; acced XIX, 30; maeslen II, 15; segna XXI, 6; sêgni XXI, 8; segni XXI, 11.

Der umlaut eines für $a$ anzusetzenden (meist analogisch wieder eingeführten) $a$ ist $a$ (S. $\S 89,3$ ). So in der flexion von fara: 2.3. sg. faris XI, 8; farces XII, 19; fares XIII,3 etc. Ferner wohl auch bei saecga gegenüber wests. secgean: pl. ind. saegces XVIII, 29 (gesaecga opt. Mt. VIII, 4); infin. sagcganne XVI, 12. - Die häufig belegten geminationslosen formen der 1 . sg. praes. ind. (vgl. vorn unter $a$ ) sind jedenfalls junge neubildungen, ebs. bei habba, wo aber im plural meist $a$ steht (a: haebbus XII, 35); dagegen gehört der opt. hierher: hoebbe 188,10; pl. hoebbe X, 10; XII, 36 etc.; s. auch unter stammbildung der schw. vb. und Bülbring, Anglia, Beibl. IX, 90-94; dazu noch nafge IX, 8; nofigum XII, 5; XIII, 29; s. Sweet, St.A.D.

Als lehnwort ist hier zu nennen: loeddin XIX, 20; loedinisc XIX, 20; loetin XX, 24 rd.

Vielleicht ist auch so zu erklären das $a$ in ongagn XIII, 18; togagnes IV, 51; -as XI, 20 (doppelformen *gagan - *gegin; vgl. auch Dieter II, p. 771); (formen mit eae s. unter wirkung vorhergehender palatale).

Westgerm. $a$ vor nasalen $(=q)$ hat als umlaut $e$ : acenned Prf. 3,14; bremmde Xl, 33; tobrengende Prf. 6,16 (s. S. $§ 407$ anm. 15); brengo XIX, 4 etc., ende Prf. 1, 12; ênde XIII, 1 ; feng Prf. 8, 9; ondfengo VII, 39; leng Prf. 5, 10; menigo VII, 31.43 
(subst.); menigo XII, 9.12 (adj.) (daneben formen ohne umlaut s. unter vokalwechsel); genemned Prf. 3,9 ; gesendet Prf. 3,5; gesendes XVII, 8. 25 etc.; unascended Prf. 1, 12; penninga VI, 7; stenc XII, 3; stenceð XI, 39; オencas XI, 50; オene I, 33; V. 43 etc.; (Jcene XIII, 20); s. Streitberg § 187.

a findet sich nur in ungewammed Prf. 1,6; unawammed Prf. 1, 12 (unawoemdo Mt. XIX, 12) vielleicht unter dem einfluss des $w$.

Lehnworte: embehtes XII, 26; embehtmonn XX, 24; embehtmenn II, 9; XVIII, 12.18 etc. (ambehtmann XX, 25; formen mit $a$ s. unter a); engel V,4; XII,29; englis XX,16 rd.; englisc 188, 7 (formen mit $a$ s. unter a); cempo XVIII, 12. Das bei ausfall des nasals vor stimmlosem reibelaut aus $a$ entstandene $\bar{o}$ hat als umlaut oe: geoehtadon $\mathrm{V}, 16$; oeðað III, $8<{ }^{*}$ anpjan.

Westgerm. $a$ vor $r$-verbindungen (=ws. $e a$ ) hat zum umlaut $e$ : gecerredo Prf. 1, 10: ymbcerde Prf. 3,11; dernunge XVIII, 20; dernelegerscip VIII, 4; gere XXI, 12; gegeruiga XIV, 2; gegerelum XX, 12; gerde XIX, 29; unawerded Prf. 1, 12.

a findet sich in uarmde XVIII, 18. 25 (uearmdon XVIII, 18) wahrscheinlich unter dem einfluss des $w$; ebs. in warc Prf. 2, 3 (dolor, an. verkr, jo-stamm).

Westgerm. $a$ vor $l$-verbindungen schwankt in seinem umlaut zwischen $a$ und $e$.

ae begegnet in aeldo IX, 21; celdo IX, 23; celdum Prf. 5, 7; 6,13 (comp. für aeldrum); (die formen mit $a$ bei Mt. und L. erklären sich durch anlehnung an den plural von aldor); auceled $\mathrm{XX}, 1$ (zu ws. wielwan); ucelle IV, 6; ucella IV, 14; uaelle Prf. 4,4 (formen mit $e$ s. unten);

vor der sekundärverbindung $l c$ in huselc Prf. 5, 13; V, 12; VI, 51 etc (Mt. Mk. L. J. zs. 30); sucelce Prf. 2, 3; 4, 9; I, 32 etc. (Mt. Mk. L. J. zs. 18); S. $§ 43$ anm. 4 (formen mit $e$ s. unten, mit oe unter $w$-einfluss);

$e$ kommt vor in huelc I, 19.22; II, 25 etc. (Mt. Mk. L. J. zs. 50); suelce I, 14 etc. (Mt. Mk. L. J. zs. 41); uele IX, 7; lifwelle IV, 10 (form mit oe s. unter $w$-einfluss).

Ein hierher gehöriges lehnwort ist felle- (read) XIX,5; felle-(readum) XIX, 2, das in volksetymologischer umbildung für palle- (lat. pallium) eingetreten ist, s. B.-T. unter pallen = purpureus. Vgl. in dieser bedeutung auch felle L. Prf. 9, 2. 


\section{Umlaut von 0 .}

Ueber die bedingungen dieses umlauts s. S. $\$ 93$ anm.; der $i$-umlaut des (analogisch wieder eingeführten) $o$ ist $e$ : exin II, 14. 15; mérne I, 43; gibêlde? 188, 3; vgl. Skeat VIII, anm. 8.

Ueber das lehnwort celmertmonn $\mathrm{X}, 12.13$; s. L. $§ 13$ note.

\section{Umlaut von $u$.}

Der $i$-umlaut des westgerm. $u$, an dessen stelle vor $a$ der folgesilbe $o$ getreten ist, S. $\S 93$ anm., ist $y$ : byrig Prf. 2, 1; bebycgendo II, 14; bydle IX, 31; bydno II, 6; bybyrge XIX, 40; gebyred II, 4 rd.; gebyrelico IV, 9; cyninge Prf. 4, 15; cynig I, 49; cynn XI, 48; gecnyht XI, 44: cymed I, 30; opt. cyme Prf. 5, 6 (s. Kluge, P. G. $\S 166 ; y$ auch in 1. sg. und den pl. des praes. ind. eingedrungen; s. unter stammbildung st. vb. IV); dynt XVIII, 22; fyrhto VII, 13; fylgendo Prf. 3,8; gefylde Prf. 4, 15; fyllnisse I, 16; ymbgyrde XIII, 4; forhycganne Prf. 7, 12; hyhtas V, 45; hyncgreð VI, 35; eftgemyndig Prf. 7, 16; genyht VI, 7; smyltnisse VI, $16 ;$ snyttro 188, 11; spyrdo VI, 19; onscynað XIV,27; gestyredo Prf. 6,17; synnum Prf. 1,7; syndrige II, 6; syndrigo X, 4 etc. (y Mt. Mk. L. J. zs. 23; daneben ui, uy, $w i, w y, u$, uu S. unten); symbel VII, 2. 14; symbeldage XI, 56; getrymeð Prf. 4, 3; getryccað XVI, 33; ठyrsteð IV, 14; ठyrnenne XIX, 5; giðyngo 188, 11; wyrd Prf. 5, 10; towyrpnisse VII, 35; wyrtcynn XII, 3; gewyrces Prf. 3,3; gewyrca Prf. 4,15 etc.; s. S. $§ 164,2 ;$ yflo Prf. 4,11; ymb $48 \mathrm{mal}$; ym Prf. 3,13 ; ofergylded 188,5 .

Ueber $y$ in scryngcara V, 3 vgl. Bülbring, E. St. XXVII, 84.

Die von Sievers, Beitr. XVI, 236 gegebene erklärung des $y$ in dyde IV, 29; VII, 21; XIII, 12 etc. (daneben formen mit $\bar{e} \mathrm{~s}$. unter $\bar{a}$ sowie unter verba auf $-m i$ ) und styd VI, 10; X, 40 stösst auf schwierigkeiten, da das germ. in der reduplikation stets echtes $\breve{e}$ aufweist und die ei/oj-stämme nie schwundstufige wurzelsilbe hatten, vgl. Kluge, P.G. § 102, p. 402 ;

ui für $y$ findet sich geschrieben in suindriga Prf. 2, 5 (Mt. Mk. L. J. zs. ui 8, swindrigum Mt. Prf. 4,1; swyndria Mk. XIV,19; $s^{u}$ yndriga Mt. IX, 1; suyndriga L. Prf. 3, 7 - bloss $u$ (uu) für ui? - suundor VII, 18; sundur XVI, 32 (Mt. Mk. L. J. zs. 4); fuilgendo XI, 31 ;

$i$ begegnet bei drihten Prf. 1,9; 3,8; 4, 6 etc.; vgl. Bül- 
bring, Anglia, Beibl. X, 7; gifillid XIX, 28; getri(m)að Prf. 7, 17; birilum II, 5; bisen XIII, 15;

$e$ findet sich in endebrednisse Prf. 2,4;8,1; s. L. § 27,2; Zeuner $\S 30$; S. $\S 180$; ondesne XIX, $38=$ ws. ondrysne?

Lehnworte: cyrtel, cyrtil XIX, 23; mynetro II, 14; pytt IV, 11; gescyrte Prf. 3,12.

IV. Der umlaut von $\bar{o}$.

Westgerm. $\bar{o}$ hat als umlaut oe: boec Prf. 1,13; bôec XX, 30; eftgeboetad III, 4; bloedsade VIII, 48; bróema, bóecere XIX, 37 rd., doeg Prf. 1, 14, sunnedoeg Prf. 4, 9; s. S. § 288 anm. 1; § 289 anm. 2; doema Prf. 3,15; gedroefedo Prf. 6,1; foerde VIII, 1 ; foedendo Prf. 8, 11; foed XXI, 15; foêd XXI, 16; foet Prf. 6,11; foêt XI, 2; gefoerum XI, 16; gefroefrende XI, 19; gloedum XVIII, 18; gloêdi XXI, 9; ingemoetet Prf. 2, 3; gemôete XIX, 4; gemoêt XIX, 6 ; oeđle IV, 44; oeđiluald 188, 12; giroefa XVIII, 28; groêfa XIX, 4. 38; soecað I, 38; smoeðe XIX, 23; auoeded X, 20; uoepende XI, 33; woerig IV, 6; uoestern Prf. 3,5; I, 23;

$e$ kommt vor in bledtsigas XIII, 13 (wohl verkürzt); Eðiluald 188, 2; gerefa XIX, 22 s. oben; hel XIII, 18, s. S. § 222, 2.

Lehnworte: oferglôesade 188, 7; hierher zu stellen ist auch floege VI, 22 (= "naviculum"; Hilmer p. 30 verzeichnet sonderbarer weise "musca" gegenüber dem lemma des textes), das offenbar ein nordisches lehnwort ist: *flaujom > isl. fley, norw. floy, dän. *flö (s. Noreen p. 304) findet sich in der bedeutung "navigium" Heilagramanna sögur udgivne af C. R. Unger, Kristiania 1877: II, 425, ${ }^{6} ; 430,17 ; 447,{ }^{6}$; Fornmanna sögur Kjebenhavn 1825-35: VIII, 209, ${ }^{16} ; 211,9$ (nach Joh. Fritzner: Ordbog over det gamle norske sprog, Kristiania 1886). Mit der erhaltung des intervokalischen $j$ stellt sich das wort zu cēga u. a.; das umgelautete oe entspricht einem $\bar{o}$ in brydlopum Prf. 1,3 ; ora 188,$9 ;$ s. unter $\bar{\delta} \S 9 ;$ S. $\S 26$ anm.; vgl. auch Whitney, C.D. unter fly-boat.

oe ist auch der umlaut des vor nasal zu $\bar{o}$ gewordenen westgerm. $\bar{a}$; s. S. $\S 68$ anm. 2 ; uoenas XVI, 2 ; woende Prf. 8,4 etc.; gecuoemo VIII, 29; woen IV, 10; daneben wênde XX, 15; wenes Mt. XVIII, 1.

Ueber den $i$-umlaut des westg. $\bar{\alpha}=$ angl. $\bar{e}$ s. unter $\bar{a} \S 6$.

V. Der umlaut von $\bar{u}$ -

$\bar{u}$ wird durch folgendes $i$ zu $\bar{y}$ umgelautet: bryd(lopum) 
Prf. 1. 3; bryd(guma) Prf. 4, 1; gebyde I, 14; S. § 416 anm. 11d; gedrygde XI, 2; dryga XIII, 5; giðryde 188,3, vgl. auch S. § 117 anm. 1 ; $\$ 408$ anm. 18; untynde Prf. 5, 10; fyr XV, 6; s. K. L. p. 75 .

Ebenso ist $\bar{y}$ der umlaut des nach ausfall des nasals vor stimmlosem reibelaut $\mathrm{zu} \bar{u}$ gelängten westgerm. $u$ : cyðnisse Prf. 1, 4; cyðig Prf. 6, 2; cyðling XVIII, 26 etc.

VI. Der umlaut von $a i$.

Westgerm. ai $(=$ ae. $\bar{a})$ hat zum umlaut $\bar{a}$ : $\operatorname{cor}$ Prf. $3 ; 3$; ae I, 17; $\hat{x} \mathrm{XII}, 34$; anigmonn I, 18; claen Prf. 3, 14; dael IX, 23; dềlo XIX, 23; gebraeded XIX, 13; hoemdum Prf. 1, 3; hoedna VII, 35; haele Prf. 3, 15; helend Prf. 4, 1; laedende Prf. 3,6; lares Prf. 2, 6; gelaefdon VI, 12; løeðठо XV, 18; mø VII, 31; gimoenelice 188, 2; roeco XIII, 26; sa Prf. 4,16; gestcena VIII, 5; sconas XIX, 36; tucem Prf. 3, 8; ðcem dat. pl. Prf. 1, 8; wrceðठo Prf. 4, 3; aelc II. 10; wniht VI, 63; naeniht I, 3; s. S. § 100 anm. 3;

$e$ stets in eghuelc III, 8.15 etc. (nur aghuelc Mk. XIV, 19; aghuoelcum L. Prf. 2,9); S. § 100 anm.3; § 347 anm.2. 3; enne Prf. 5, 3; 6, 10 etc. (anne nur Mt. Prf. 14, 14); geneolecde Prf. 4, 14; II, 13 (geneolicde Prf. 3, 12 wohl mit anlehnung an die bildungssilbe -lic). In den beiden letzten silben ist verkürzung eingetreten, vgl. S. $§ 324$ anm. 1; erendureca IX, 7, s. unter $\bar{a} \S 6$.

Lehnworte: casares XIX, 12 ( $a$ bei Mt. Mk. L. J. zs. 7); caser XIX, 12.15 ( $a$ bei Mt. Mk. L. J. zs. 16), vgl. Pogatscher $\S 199$ ); Jral VIII, 34; Jrael Prf. 5, 16, s. Kluge, NSt. § 18.

Umlaut von alte. $\bar{a}$ liegt auch vor in ofra I, 18; nofra $\mathrm{XIX}, 41$, wenn die etymologie $\bar{a}$ in feore richtig ist.

\section{Der umlaut von au.}

Als umlaut des aus westgerm. au entstandenen alte. $\bar{e} a$ erscheint $\bar{e}$ : gebêcnade XIII, 24; gebegdum XIX, 30; gedeðet XII, 33; degelnise Prf. 3, 14; degle VII, 4; (nach S. § 128, 3 kann auch die umlautlose form mit suffix -olo zu grunde liegen, dann ist palatalumlaut anzunehmen); cependo Prf. 3,12 ; gehenað VIII, 10; hera XII, 26; gehêrdon XIV, 24; hefid-(ponna) XIX, 17 (s. unter vokalwechsel); hreme XI, 31; hrêmende XX, 11; gelefanne Prf. 4,2; gelêfae XVII, 20; leđrede XI, 2; ned-(ðcerf) Prf. 3, 12; neteno IV, 12; receles XIX, 39; fiftenum XI, 18 (aus -ea- $\mathrm{s}$. unter kontraktionen); gesêne Prf. 3,11, s. S. § 222; vielleicht ist als 
grundform statt *sahnia vielmehr *sa(g)wnia anzusetzen; togêcde Prf. 5, 13.

ae findet sich bei caepinces II, 16: gehone VIII, 6; gehoenas VIII, 10 (in allen anderen formen $e$ s. oben).

Für $\bar{e}$ vor palatalen konsonanten ist oft $e i$ geschrieben, vgl. north. diphthonge § 14 . So stets bei ceiga: ceigeð Prf. 4,12; 6,12; ceigde XI, 28; XVIII, 33 etc.; heig VI, 10; deigelnise VII, 4; deigle XIX, 38.

\section{Umlaut von eu.}

Das im westgerm. vor $i, j$ der folgenden silbe aus $e u$ entwickelte iu erscheint weiter umgelautet $\mathrm{zu}$ io (geliornisse Prf. 1,14; oferlioraes V, 24; s. § 18d); lioda Prf. 4, 5; niua XIII, 34 (zur schreibung vgl. unter $w$-einfluss); Jiostrum Prf. 1, 7; diorwyrde XII, 3.

$\S$ 18. Der $u$ - und o/a-umlaut.

Der $u$ - und $o / a$-umlaut erstreckt sich auf die vokale $e, i$ und ist bei diesen regelmässiger durchgeführt als im ws. Seine geltung ist eine verschiedene, je nachdem die umlautswirkung von einer ableitungs- oder einer flexionssilbe ausgeht. Namentlich soweit er von o/a, das meist flexionssilben angehört, erzeugt wird, erfährt er durch ausgleichungen vielfach störung.

Gewöhnlich tritt der umlaut nur vor einfachem konsonanten ein, doch findet er sich vereinzelt auch vor konsonantgruppen, s. unten, vgl. S. $\S \S 101-107 ; 160$.

a) $e>e o$ durch ein ursprüngliches $u$ der ableitungssilbe, auch wenn sich dessen qualität geändert hat oder später synkope eingetreten ist: heofun 1,51 ; heofoncund VI,31 rd.; heofnum Prf. 4, 18; 6, 18; seofanum Prf. 8, 9; seofunda IV, 52 .

ea begegnet in compuearod XVIII,12 (neben uorud XVIII,3, s. unter $w$-einfluss); s. S. $\S 150$ anm. 1.

b) $e>e o$ durch ein ursprüngliches $u$ der flexionssilbe: feolo VI, 11; XI, 47 (fealo L. XII, 48); bigeonda XIX, 18, s. Bülbring, Anglia, Beibl. IX, 99 - hier wirkt der umlaut über eine konsonantengruppe hinweg -; heono I, 29. 36 etc.:

$e a$ weist auf geafa Prf. 3,4 ; IV, 10; XVI, 2; daneben ohne umlaut gefo I, 17; spearum XVIII, 3, wenn nicht spearcum zu lesen ist, s. Cook; begeande VI, 25.

o/a-umlaut liegt vor bei gebeara XVI, 12; daneben imp. pl. 
berad XXI, 10; eatas XXI, 12; eattanne VI, 52; daneben ettas L. V, 30; ettanne Mk. V, 37; ongeattas Prf. 5, 14; begeatta Prf. 7,11 ; ongeatas $\mathrm{X}, 14$ - hier findet sich keine umlautlose form daneben; Jageana II, 10; geane IV, 35; dageone XI, 30.

Schliesslich gehört hierher auch noch sealla XIII, 11 (seallad L. XXI, 12) etc., wo, wie schon Lind. $\$ 9, \mathrm{I}, 2 \mathrm{~b}$ vermutete und Bülbring, Anglia, Beibl. IX, 96; E.st. XXVII,87 näher ausgeführt hat, nicht brechung des $a$ vor $l l<l j$, sondern sekundärer $o / a$-umlaut des $e$ stattgefunden hat, die gleiche entwickelung zeigt sich in gecearredo VII, 53, wo ea in ähnlicher weise eingeschleppt wurde wie bei sealdes XVII,4.11, s. $\S 16,2$; vielleicht auch geondsuearade II, 19; V, 11; geondsuearede XVIII, 23; vgl. S. $\S 416$ anm. $13 \mathrm{c}$.

c) $i>$ io durch ein ursprüngliches $u$ der ableitungssilbe: cliopade VII, 37; gelionade XIII, 12 (daneben gehlinade L. XXII, 14); gismioðade 188, 4; điosue IV,12, vgl. S. § 105 anm. 8.

d) $i>$ io durch ein ursprüngliches $u$ der flexionssilbe: $g e$ uriotto Prf. 4, 12; wriotto V, 39 (daneben writto Mk. XIV, 19; wuritta Mt. Prf. 1,2); scioppo VI, 23. 24 (daneben scipo Mk. IV, 36); liofo VI, 57; XIV, 19; flioton IX, 22; fordriofon IX, 34 (daneben fordrifon Mt. VII, 22); gegrioppo VII, 32.

o/a-umlaut liegt vor in hiona II, 16; XIV, 31; XIX, 18; hiora III, 19; VII, 53; clioppað I, 15; clioppendes Prf. 3, 5; I,23, s. S. § 370 ; hier niemals formen ohne umlaut; underniomane Prf. 8,2; genioma X, 29; imp. pl. niomad XI, 39; daneben ind. pl. nimað XX, 23; niomende II, 6; wriottana Prf. 2, 4; gegrioppa VII, 44; hrioppad IV, 36 ( $\zeta \mathrm{im}$ alten ablaut $\mathrm{zu}$ ej vgl. brukan, lukan); hierher wohl auch geliornisse Prf. 1, 14; oferliorces V, 24; vgl. S. § 382 anm. 3; § 384 anm.3; giosterdoeg IV,52, s. S. § 160, 3; hier wirkt der umlaut über eine konsonantengruppe hinweg.

ia begegnet in gegiauað Prf. 7, 6; vgl. S. § 416 anm. 14 b;

ea in neadan VIII, 23; heana VII, 3;

eo in heona XVIII, 36; oferleorað Prf. 4,10; oferleora XIII,1, s. oben zu geliornisse.

Hierher sind auch zu stellen seulfres 188,9.10; und mit accentverschiebung und schwond des $i$ suulfre 188,5 ; sonuuald VI, 31 rd.; soðða VI, 16; XI, 7 etc.; vgl. Bülbring, Anglia, Beibl. IX, 95; E. St. XXVII, 88. 
$\S 19$. Sog. palatalumlaut.

Ueber diese erscheinung ist von Bülbring, Anglia, Beibl. VII. IX. pass. X 1-12 eingehend gehandelt worden. Dieser unterscheidet zwischen einer eigentlichen palatalisierung gewisser vokale und diphthonge vor $h t, h s, h b$, wenn darauf nicht unmittelbar ein vokal velarer art folgt und einer monophthongierung derselben laute, für die er a.a.o. X,3 fussnote den namen "ebnung" vorschlägt. Doch lässt sich diese scheidung in unserem denkmal kaum durchführen, da einmal das material nicht reichhaltig genug vorliegt, andrerseits in den flexionen weitgehende analogische verschiebungen zwischen velaren und palatalen (endungs-)vokalen eingetreten sind, sodass ich es vorziehe, die einzelnen belege nach der von Sievers $\S \S 161-165$ gegebenen einteilung anzuordnen.

I. ea wird $\mathrm{zu}$ oe vereinfacht vor $h, h t, x(=h s)$ : gesceh I, 18. 32. 34. 50 etc.; gesaeh I, 48; ahtuu Prf. 8, 6; chtu 188, 9; whtuo $\mathrm{XX}_{,} 26$; tceherende XI, 35; moeht Prf. 6, 5; I, 12 etc.; moehte IX, 33; XI. 37; moehtun Prf. 5, 5; moehton VIII, 6; XII, 39; frex XI, 2.

Vor $h s$ findet sich einmal $e$ in auexe III, 30; doch haben hier Mt. Mk. L. stets $x$.

Das $e$ in embeht, neben dem wohl $i$, aber nie $a$ belegt ist (s. L. § 18,2) gehört kaum hierher; es ist vielmehr sekundärer suffixablaut $0: e$ anzunehmen, wodurch sich auch der $i$-umlaut der ersten silbe erklärt (neben ambeht XXI, 23); $e$ ist dann eine abschwächung aus $i$ in minderbetonter silbe.

$e a$ vor $r c$ erscheint als $e$ : (forueard)- mercunga Prf. 3,1 (ws. mearcung); gemercade III, 33.

II. $e a$ wird vor $h, c, g$ zu $\bar{e}$ : heh IV, 11; hehstald Prf. 1, 2.5 etc.; becon Prf. 1,7; IV, 54; beceno IV, 48; VI, 2. 26; ge. brecon VI. 26; gebrêcon VI, 58 (mit einschleppung des singularvokals in den plural praet.); gebrêicon VI, 31, s. § 14; lecuord Prf. 8, 4; lehtun XVIII, 1; sigbeg XIX, 2; fromgebeg V,13; gebêg XX, 5; ego IV, 35; êgum XII, 40.

a kommt vor in gefleeh Prf. 4, 15 (daneben gefleh Mt. Prf. 16,4); überwiegend bei $a c$ Prf. 3,5; 7,5; $\hat{x} c$ XII, 10; XIV, 27 etc.; eacc VII, 47; ecae IX, 27 (für eacc?); ê̂e XII, 2; XIII, 9 (a, $\hat{x}, e c e, e \hat{e}$ Mt. Mk. L. J. zs. 115; ec XVI, 3; XIX, 6; êc XIII, 34; 
XVII, 14. 16. 19. 26 etc.; $e, \hat{e}(e \hat{e})$ Mt. Mk. L. J. z. 54); daneben auch eac Prf. 1, 11; III, 23; XI, 5. 48, s. S. § 163.

Im wechsel mit $a$ findet sich $a$ bei $\partial c h$ VIII, 16; X, 38; ðacch IV, 2 etc.; бah VIII, 14 (Mt. V, 29; XVI, 26; Mk. IX, 50; L. XVIII, 4); auch einmal ðeah Mk. III, 26. Bei den letzten beiden formen nimmt Bülbring, Anglia, Beibl. IX, 100 verkürzung an.

Ist $a$ in ofgoet II, 15 vielleicht nach S. $\S 108 \mathrm{zu}$ beurteilen? vgl. auch $\S 12$.

III. $e o$ wird vor $h, x(=h s)$, ht und $r c$, rh sowie $c, g$ zu $e$ : geseh (imp.) I, 46; XI, 34; XX, 27 (gesagh VII, 52; gesih Mk. I, 44; XIII, 1 ist eine analogische bildung nach der 2.3. sg. ind. praes.); feh 188, 5; sex Prf. 6, 11; II, 6. 20; XII, 1; reht VI, 32; fehta XVIII, 36; uerc VI, 29: uerco VI, 28; VIII, 41 (sonst mit oe s. unter $w$-einfluss; über warc Prf. 1,6; s. Bülbring, Anglia, Beibl. IX, 73 fussnote); berhtne XII, 28; berhtnade XII, 28; gebrehtnis Prf. 6,15; gebrehtnige Prf. 6,17; derh Prf. 1, 12; 2, 5 etc. (einmal ðorh XIX, 34), das wohl nicht wie bei Lind. $§ 11$

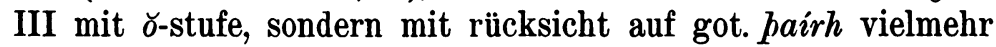
mit $e$-stufe im gegensatze zur ws. schwundstufigen bildung anzusetzen ist; erendureca IX, 7; recone $\mathrm{V}, 9 ; \mathrm{XI}, 31$; spreco IV, 26; lehnwort regles Prf. 1, 12; vgl. S. § 164 anm. 2.

$a$ findet sich in cnceht VI, 9; XVI, 21; cncehtas XXI, 5; vgl. S. § 164 anm. 1; Bülbring, Anglia, Beibl. IX, 72. 73; X, 1 .

IV. éo vor $h, h t$ wird zu ex: neh III, 23 (< angl. *nēoh= ws. néah vgl. geneolicde $\S 16,3$ ), fle/as X, 5 (mit rücksicht auf fliid $\mathrm{X}, 12.13 \mathrm{~s}$. unten ist wohl so zu trennen; vgl. auch unter kontraktionen); leht Prf. 1,6; 5, 10 etc.

Eine palatalisierende wirkung ist vielleicht auch ausgegangen von -st in brest XXI, 20; doch vgl. § 13 .

V. io wird vor $h, h t, h \delta, r c, r g, g, c$ zu $i$ : betuih Prf. 3, 10; bituien IV, 33; XIII, 22; S. § 165 anm. 2; noniht I, 3; sighðana Prf. 1, 11; wircanne II, 4 rd.; wircað II, 5; geuirce, uircas III, 2; S. § 164, 2; gebirged VIII, 52; tuiggum Prf. 6, 12; tuiggo XV, 5; sticadun XIX, 37; ofstigon VI, 16.

$e$ findet sich bei rehtas I, 23; gewerco VI, 28 wohl in anlehnung an das adj. bezw. subst.; vgl. S. § 164,2 anm. 4; ebs. ingeberigde II, 9, wo, wie unmittelbar danebenstehendes ingebarg 
beweist, wohl vermischung mit dem st. vb. III bergan (= ws. beorgan) stattgefunden hat.

VI. $\bar{\imath} 0$ wird vor $h, x(=h s)$, ht $\mathrm{zu} \bar{\imath}$ : fliið $\mathrm{X}, 12$; s. auch kontraktionen; lixende V, 35; inlichtade Prf. 6,1; inlihted I, 9.

Bei frühzeitigem ausfall des $h$ vor (stimmhaftem) konsonanten unterbleibt die vereinfachung: heannissum VIII, 23; geneolecde Prf. 4,14; eorodmonna XVIII, 12 rd.; vgl. § 16, 3.

$\S 20$. Die einflüsse des $w$.

Unter dem einflusse eines vorausgehenden $w$ wird oft:

I. $\ddot{~} \mathrm{zu}$ ое: сиоељо IV,35; VI,47 etc.; cuoeðestu I, 22; cuoeðas IV, 35; VIII,54; gecuoeden Prf. 4.1; cuoedendo Prf. 4,כ (cuoceðaठ L. VI, 26; varianten der orthographie s. bei Paul, Beitr. VI, 38; formen mit $e, a, o$ s. unter stammbildung der st. vb.); uoeg Prf. 7,4; XIV, 6; huoelc XI, 9 (huelc)- huoegu I, 46; eghuoelc XI, 26; suoester XI, 1; suuoeðles XI, 44; geuoefen XIX, 23; uoerc Prf. 3,16 ; IX, 3 - hier hat offenbar der $w$-einfluss nach dem eintritt des palatalumlautes raum gewonnen.

II. \& zu oe: auoecce Prf. 6,9; auoecco VI, 54; uoel V, 4; cuoellanne V, 18; auoergado VII, 49; owoerigdon IX, 28; ymbuoende XX, 14; tuoelfum Prf. 5, 3 .

III. $\bar{e}(=\mathrm{ws} . \bar{\alpha}) \mathrm{zu} \overline{o e}$ : cuoedon $\mathrm{I}, 22$; cuôedon XIX,6; uoeron Prf. 6,1; XV,27; woere Prf. 1, 6; 3, 3. 16; woero XI, 19; XIX, 31; huoer XI, 32 (vgl. §6); uoedo XIII, 4; woepnum XVIII, 3.

IV. कe $\mathrm{zu}$ oe: cuoed Prf. 3, 14; III, 7; сиоюе Prf. 5, 11; 7,12; XI, 11. 51 (coed XI, 40; XVIII, 37; mit verlust des $w$, z. orthogr. s. oben); huoeðer VII, 41; huoeðre Prf. 2, 4.

In auoehte Prf. 4, 10; 6, 8 ist aus ea vor ht vereinfachtes $a$ dem $w$-einfluss unterlegen; vgl. S. $\S 162$ anm. 3.

$V$. eo (aus brechung oder $u$-, o/a-umlaut) zu $o$ (über das verhalten der einzeldialekte s. Sievers, Beitr. XXII, 255. 56): cиоðаð Prf. 4, 20 (bei diesem vb. sonst gewöhnlich oe direkt aus $\ddot{e}$ entwickelt, s. I); dagegen stets wosa I, 12; uôsa XX, 27; uosað XVI, 4 (diese eigentïmliche doppelheit erklärt sich wohl daraus, dass bei cuoeða die formen mit altem $\ddot{e}$ in der mehrzahl sind und die übrigen, denen lautgesetzlich $w o(<w+e o$ durch o/a-umlaut) zukam, in ihrer lautgebung beeinflusst haben, während neben dem regelrecht mit $o / a$-umlaut gebildeten inf., uosa $\left(<{ }^{*}\right.$ weosan $)$ keine anderen formen stehen, wo unumgelautetes 
$\ddot{e}$ direkt zu oe hătte werden können); uorpo VI, 37; uorulde IX, 32; uorud XVIII, 3; suuord XVIII, 10; suôrd XVIII, 11; uôrðe XVIII, 15; S. § 72; geworðad Prf. 6,2; worðe Prf. 6, 12; huothuogu IV, 33.

VI. ea zu $a$; vgl. S. $\S 160$ anm. 2: ward (praet.) Prf. 2, 2; awarp Prf. 3, 12; geonduarde, geonduardon $\mathrm{X}, 33$. 34; duruuardae XVIII, 16; uarðe (subst.) XXI,4); hierher gehört auch farma (ws. feorm) Prf. 6, 19; XIII, 2.4 (farmo XXI, 20; $a$ Mt. Mk. L. J. zs. 13 ; a 10); vgl. fattro Mk. V, 4; farra L. XXIII, 49; s. L. $\S 13,3$.

VII. io aus brechung oder $u$-, o, $a$-umlaut $\mathrm{zu} u$ : untetlice Prf. 3,6; gewöhnlich uut'; uuton III,2; süopa II,15 (für swupa?); wuducynn XIII, 3.

Bei hinzutretendem $i$-umlaut entwickelt sich $w u$ weiter zu wy; S. § 156, 4: arwyrðe Prf. 4, 10; wyrse V, 14; wurresta, uirresta II, 10 u. rd.; vgl. S. § 72 anm.

VIII. Die gruppen ěow und tow werden oft $\mathrm{zu} e w(e o, e u)$ und iw (io, iu) vereinfacht, vielleicht nur graphisch verkürzt; S. § 156, 5 anm. 5: feuor XI, 17; feuoer XIX, 23; feoer (doger) XI, 39; feuero IV, 35; feortig II, 20 (daneben feouer 188, 10); treum XIX, 39; getrewad XVI, 33; euunge VII, 10; X, 24; aed-, eude V, 20 (über eawunga, eawan s. unter $w$ ); niua Prf. 1, 10; niuces Prf. 6,5; niunge III, 3; iuerro IV, 35; iure Prf. 5, 12; iuih Prf. 5, 15; giues IV, 9; giuad XVI, 24. 26; gewigende Prf. 6,17 (gcwigende? s. Skeat's text p. 6 anm.1); diul VII,20 (diwle Mt. XXV, 41; L. XIII, 32; daneben diubul, diowlas etc. s. unter kontraktionen).

Bemerkung. $w$ schwand, indem es folgendes $i$ zu $y$ verdumpfte in nyste I, 31. 33; nyston $\mathrm{X}, 5$ etc.

\section{$\S 21$. Wirkung vorausgehender palatale.}

I. Anlautendes ursprüngliches $j$ (geschrieben stets $g$ ausser in eigennamen) unterscheidet sich in seiner einwirkung auf folgende vokale mehrfach vom ws. status. So erscheint für ws. ge (=ihr) meist gie Prf. 4,16; 5, 13 (gice L. XI, 42; ie Mt. Mk. L. J. zs. 685); ge IV, 21; VIII, 36; gê XIX, 36; XX, 23; gee III, 12 (im ganzen nur 192; gae Mk. XIV, 42; dagegen für ws. giet erscheint get Prf. 5, 5; II, 4; III, 24 etc.; gett XII, 35; XIV, 19; XVI, 12; daneben gat VII, 33; für ws. giu, geo erscheint 
gee IV, 35; gêe XIV, 19; geê XVI, 16 etc.; gi? XVI, 32 rd. (giee Mk. XV,44).

$u$ ist diphthongiert $\mathrm{zu}$ iu in giungra XXI, 18 (daneben giugesta L. XXII, 26; ging Mk. XIV, 51).

Gegenüber ws. gear erscheint geres XI, 49; XVIII, 13; gerc $\mathrm{V}, 5$; wo sich vielleicht urangl. $\bar{e}$ unverändert erhalten hat; doch s. S. $\S 157$ anm. 1.

II. Nach $g^{\prime}, c^{\prime}$ wird $a$ (= westgerm. $a$ ) zu $e a$ diphthongiert, doch finden sich daneben auch häufig formen mit unverändertem vokal. Für ea tritt oft die schreibung eae ein: ongeagn Prf. 6,10 (vgl. auch $\S 17, \mathrm{I}$ ); ongeat I, 10; IV,1; aedgeadre XXI,2; cedgeadre XXI,13; gegeadredon Prf. 6, 10 (aetgaedre XX,4; Bülbring, Anglia, Beibl. IX, 76/77 nimmt bei diesem wort $u$-umlaut an und zwar durch folgende wechselwirkung zwischen adverbium und verbum: *gaedyri $-{ }^{*}$ gadurōjan : $>{ }^{*}$ gaedyri *godurōjan (mit analogischem $a$ ) $>{ }^{*}$ gaedyri - ${ }^{*}$ geadurōjan (mit $u$-umlaut des $a$ zu ea) $>$ geadrian - geadre (mit analogischem ea). Einmal sind solche analogische beeinflussungen zwischen zwei verschiedenen grammatischen kategorien doch etwas unwahrscheinlich (vgl. nhd. ich se(h)e, er sie(h)t, aber gesicht); dann bleiben ongecegn, ongeat $u$. a. immerhin daneben bestehen.)

Als lehnwort gehört hierher: ceastra XI, 1; XIX, 20 (coestre IV, 5; ea Mt. Mk. L. J. zs. 75; $\propto 2$ ).

III. Nach $s c^{\prime}$ wird folgender vokal häufig diphthongiert: a zu ea: scealde IX, 22; morsceaðe XVIII, 40; frumsceaft Prf. 1,$12 ;$ sceaðana, scceððana $\mathrm{XX}, 25$.

$\bar{a}$ (=westgerm. ai) zu éa: asceadan Prf. 8,1; sceceðXVIII,11.

$\bar{o} \mathrm{zu}$ éo: sceoea L. III, 16; sceoe L. X, 4; gesceoe Mt. III, 11; aber scoes I, 27; scoe Mk. I, 7.

Eigentümlich ist das verhalten von $\bar{e}(=$ ws. $\bar{e})$, bei dem nach $g, c$ eine diphthongierung im allgemeinen nicht vorkommt; hier stehen sich gegenüber: sceacere $\mathrm{X}, 1$; vgl. S. $\S 157,2$; andrerseits scip $\mathrm{X}, 3$. 4.12.16; scipo X, 3; scip X, 4, 8; scipo Prf. 8, 11; II, 14. 15 etc.; s. Bülbring, Beibl. IX, 98.

V. Regelmässige diphthongierung zeigt geongan, geong: geonga XI, 16; geong Prf. 4, 9; geongende I, 36; geonge VII, 33; ford-geong 188, 11 etc.; einmal giungo VIII, 21.

VI. $\ddot{e}$ erleidet keine diphthongierung; doch scheint ihr $e$ 
zu unterliegen in begienda III, 26; s. Bülbring, Beibl. IX, 99. Vielleicht gehören hierher auch die $i$-formen im sing. und praes. von sealla: silo X, 28; silio XVII, 2; XXI, 13 etc., wo unter der palatalisierenden wirkung des $s \&$ zu $i e$ und mit accentverschiebung $i$ wurde; s. Bülbring, Beibl. IX, 96; E. St. XXVII, 87, s. auch unter stammbildung schw. vb. I und vgl.Dieter II,p.771/73.

Nach $s c$ wurde $o$ auch diphthongiert im lehnworte gesceortade II, 3.

$\S 22$. Hiatus und kontraktionen.

I. Ursprüngliches $a h+$ vokal ergiebt meist $\bar{e} a$ neben $a(a)$ : gefea I, 16. 17; III, 29 (< * gifaho S. § 111, 2); geslea XVIII, 31; ealond Prf. 1, 11 (eolande 188,2, wohl mit assimilation an das folgende o); daneben aðuah IX, 7. 11 (imp. analogisch gebildet S. § 162 anm. 3; geslaa L. XVIII, 13; geslâs L. XXII, 49); auch a findet sich: sla Mt. XXIV, 49.

Ohne kontraktion erscheint tcherende XI. 35 (neben tearum L. VII, 38. 44); (germ. $h h$ ).

Eine eigentümliche bildung weisen die praesensformen einschliesslich infinitiv von *bwahan auf: neben regelmässigem $e a$ bezw. $a(a)$ (nur aðuah IX, 7.11 s. oben; бuah Mt. VI, 17; бwas Mt. XV,2) erscheint weitaus häufiger oa: aðoa XIII, 8. 10. 14; L. VII, 38; geðoas XIII, 6; Mt. Mk. L. J. zs. 7 .

Ausserdem kommt vor geðuoa L. VII, 38; đuoas XIII, 8 . Eine bloss graphische variante in der bezeichnung für $w$ liegt wohl kaum vor; die formen erinnern in ihrer bildung an hoa, foa, doa.

II. Ursprüngliches $\breve{e} h+$ gutturaler vokal erscheint fast durchgehend als ea: gefeande Prf. 5, 11; gefeas 1II, 29; gefeage III, 14; gesea Prf. 6, 14; gesead I, 39. 51; geseas XIV, 19; getea $\mathrm{XXI}, 6$; fleas X, 5; hunteantig XIX, 39 (dazu mit $i$-umlaut vielleicht fiftenum XI, 18; nach S. § 325, abs. 2); wegen hêra IV, 12 s. S. $\S 166,3$ anm. 3 .

éo kommt daneben vor bei sueor XVIII, 13; geseom IV, 19 (gesium XVI, 22 wohl nach analogie der 2.3. sg.).

$e+u$ ergiebt $e a$ in Jea Prf. 5, 16 (deas Mt. Prf. 20, 13; 21, 8; dea Mt. Prf. 21, 1); dagegen eo in treo XV, 2 rd.; uintreo $\mathrm{XV}, 1.5$; palmtreo XV, 4 .

$e+e$ wird ẽ: ece VI, 68; êce Prf. 4, 3; III, 15. 16. 36 (êce IV, 14; s. auch \& 7); vgl. auch opt. gesee IV, 48. 
III. th + ursprünglich $i$ ergiebt $\bar{\imath}$ : gesiið Prf. 5, 17; 7, 5; gesiist I, 33; fliið X, 12. 13; vgl. S. §§ 110 anm. 1; 166, 2; 358, 2;

ih + gutturalem vokal ergiebt ia in tuiaton XIII, 22 (tuiade L. IX, 7);

io in gebiotate Prf. 7,19; doch s. S. $\S 114$ anm. 1;

$i+u$ erscheint als $i u$ und io sowie ia in hiu Prf. 8,4; $\mathrm{XX}, 15$; hia XX, 16 (hio Mt. IX, 21; Mk. XVI, 10; L. VII, 50; $\left.\mathrm{XX}, 32\left({ }^{2}\right) ; \mathrm{XXII}, 56\right)$; sio IV, 23; V, 28; XII, 12; XVIII, 17; Jio Prf. 8,2 ; IV, 52; Jiu I, 9; II, 1 ; IV, 53. ea hat meist Jrea II, 6 und rd.; Jriu Prf. 8, 9 (ea Mt. Mk. L. J. zs. 14; Jrio L. Prf. 7, 3; XII, $52\left({ }^{2}\right)$; XIII, 7. 21; đreo XXI, 11; Mt. XVIII, 20; L. XI, 5 ;

$i+e$ ergiebt $i e$ und $e$ : sie Prf. $3,4.14 ; 4,1 ; 6,4$; se Prf. vb. subst. 3,$3 ; \mathrm{XII}, 40$.

In der behandlung von $\breve{t}+$ gutturalvokal macht sich starkes schwanken bemerkbar:

io wechselt mit $e o$ in frio Prf. 5, 15; freo VIII, 36; Mt. $\mathrm{XVII}, 26$; friond III, 29; XIX, 12; friondas XV, 14. 15; friondum $\mathrm{XV}, 13$ (friond L. XI, 5 etc.; io Mt. Mk. L. J. zs. 16); freond XI, 11 (freondas L. VII, 6; Mt. Mk. L. J. zs. 11); (nur io erscheint in fiond Mt. V, 25; XIII, 25. 28. 39);

ia wechselt mit io, ie iu in friad Mk. Prf. 3,17; friende Mk. IX, 36; gefriað Prf. 5, 15; VIII, 32. 36; gefriod VIII, 33; gefiad VII, 7; XII, 25; XV, 23; gefiadon XV, 24; gefiage VII, 7 (vereinzelt auch hier gefreod L. Prf. 8,9; XII, 58; gefreonad L. I, 74); in den lehnworten diacones I, 19; diabule VIII, 44; diubul Prf. 5, 3; diobul Prf. 5, 16 (io Mt. Mk. L. J. zs. 75; iu 3; ia 5; s. auch $\S 20,8$; vgl. L. $\S 64,3)$.

IV. $\bar{o}+$ vokal wird $\mathrm{zu} \bar{o}$ : foanne $\mathrm{X}, 39$ rd.; foendo Prf. 4,13 ; onfoas Prf. 3,3 ; ahoad XIX, 6 ; gehoa, hoanne XIX, 10; doam Prf. 7, 6; doe XVII, 26; doas XIII, 15; doa Prf. 4, 15 etc.; scoes I, 27. Die endung ist hier überall analogisch wieder angetreten, vgl. S. § 110 anm. 1.

V. $\breve{u}+$ vokal bleibt unverändert in bues $\mathrm{I}, 38$; londbucend Prf. 7, 9; gleitlaut stellt sich ein in trugude II, 24.

VI. $\breve{y}+i, e$ verschmilzt zu $\bar{y}$ : giðryde 188,3 .

VII. Zusammenstoss von unbetontem + betontem vokal wird durch elision des ersteren beseitigt; so des $e$ bei buta Prf. 3,14 ; togêcde Prf. 5, 13; binna XVIII, 15; nis VIII, 44; 
geade IV, 51; nam I, 21; grournun IV, 51; nach verlust des anlautenden konsonanten bei nyste $\mathrm{I}, 31$; noht Prf. 7,9; naeniht I, 3; nallas V, 40; nastu III, 8; nutton XVI, 3; nabbad II, 3; naefdo IX, 41 etc.; vgl. S. $\S 110$ anm. 4 .

$\S 23$. Schwankungen der quantität.

I. Auslautende vokale betonter einsilbiger wörter erscheinen als lang in: nû IV,23; VIII,4; nuu IV,18; nú VIII,52; núu VIII, 40; nûu XIII, 7; nûे XV,22; nê VI, 24; hê XVI, 2;

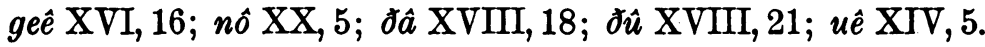

II. Neigung zur dehnung einsilbiger wörter auf einfachen konsonanten zeigt sich bei uêg XIV,5; sprêc XVI, 33; mêc $\mathrm{XV}, 25$; hîs XVI,17; uêl XVIII,23; besonders bei den en- bezw. proklitischen partikeln: ôn VII,2; XVIII, 20; 188, 4. 7; ôndattað $\mathrm{XII}, 42$; ôngeattas XIV, 7; ôngette XVII, 23; ônstiðade XII, 40; $\mathrm{XVI}, 40$; ûdedo III, 19; întrymig VI, 2; unsib VII, 43; unntrymig XI, 6; únberende XV,2 rd.; unboht XV, 25; unruh XIX, 23; ungelefen $\mathrm{XX}, 27$.

III. Dehnung vor nasal + konsonant: blind IX, 24; ofblindade XII, 40; ênde XIII, 1; hônd XIII, 9; hónd XX, 20; hûnd XVIII, 12; XXI, 8; stôndende XVIII, 16; intîng XIX, 4; pûnda $\mathrm{XIX}, 39$; gemônges XIX, 39.

IV. Dehnungen vor $r+$ konsonant: uôrd $\mathrm{X}, 21$; suôrd XVIII, 11; wôrđe XVIII, 15 (môt-sprêc)- êrn XVIII, 28; đôrnum $\mathrm{XIX}, 2$; uôrda XIX, 13; dureuêard XVIII, 17; gwurnun IV, 51; vgl. § 29.

V. Vor $l+$ konsonant findet sich nur gefâlden XX, 7 .

VI. Seltener erscheinen kurze vokale vor einfachen konsonanten in zwei- und mehrsilbigen wörtern gedehnt: fêder $\mathrm{XX}, 17$; halscôde XI, 44; hrổe XIII, 27; uôsa XX, 27; lâdanne IV, 7.

VII. Ausserdem findet sich dehnung bei êsnces XVIII, 18; bisuêठむun XIX, 40.

VIII. Als sicheres beispiel von kürzung kann wohl nur bledtsigas XIII, 13 (neben bloedsade VIII, 48, ws. bletsian) gelten; vielleicht bezeichnet die verdoppelung des nachfolgenden konsonanten verkürzung in bruccað VI, 56. 58; gebrucced VI, 50; gebrucces VI, 51; gebruccað VI, 54; vgl. ne. to shove zu ae. scüfan. Allerdings spricht die ne. schreibung brook gegen eine derartige frühe verkürzung. 


\section{B) Die vokale der mittel- und endsilben.}

Kapitel V. Vokalwechsel.

Eine aus idg. zeit ererbte abstufung der vokale tritt in gewissen suffixen zu tage (suffixablaut s. S. § $127 \mathrm{ff}$.); daneben begegnen jüngere vokalwechsel in denselben suffixen und besonders in den vorsilben sowie den schlussgliedern von kompositis.

$\S 24$. Suffixablaut und verwandtes.

I. In dem neutralen suffix os, es $=$ ae. $-r$ -

o-stufe liegt vor in doger Prf. 6,8; XI, 39; lomb Prf. 3,6; I, 29. 36; lombor XXI,15; spere XIX,34; s. S. § 288 anm.1; demgegenüber $e$-stufe in doeg Prf. 1, 14; I, 29. 35.39 etc. (zu uuldor VIII, 54 s. Kluge, N.St.B. §§ 133. 141).

Jüngere wechsel dieses suffixes begegnen in uuldor I, 14; uuldur XVII, 22; uulder XI, 40; ur im komparativsuffix -özhrađur XX, 4.

II. Vor $n$ im suffix -ono-, -eno- (part. praet. st. vb.).

Die $e$-stufe scheint erhalten in geducen XIII, 10 (glossiert locutus in verwechselung mit lotus); ahefen XII, 32, wenn dies nicht nur eine analogiebildung nach dem praesens ist; vgl. ahofen L. XVIII, 14 nach dem praeteritum. Die schreibweise der formen gedoen VI, 6; ahoen XIX, 16. 20. 32. 41; onfoen Mt. XXIV, 40 giebt über ihren lautwert keinen aufschluss; wahrscheinlich aber ist -o-en zu lesen.

Jüngerer wechsel erscheint in heofun I, 51 gegen sonstiges heofon; seofanum Prf. 8, 9 gegen seofon; linnin XX, 5; linnen $\mathrm{XX}, 6$; recune $\mathrm{IV}, 27$; recone $\mathrm{V}, 9$.

Ueber den wechsel von sprossvokalen s. unter synkope.

III. Vor $l$ im suffix -olo-, -elo-.

Von diesem suffixablaut ist betroffen das lehnwort dacccille Prf. 4,12 etc.; s. auch $\S 1.2 \mathrm{zu}$ lat. facula.

Jüngerer wechsel liegt vor bei degelnise Prf. 3,14; engel $\mathrm{V}, 4$; XII, 29 gegen deigilnisi 187, 14; birilum II, 5; micil VI, 10; XII, 24; lyttil XII, 35; Ediluald 188,2; cyrtil XIX, 23 neben cyrtel ebd.

IV. Vor $r$ im suffix -oro-, -ero-.

Jüngerer wechsel bei suundor VII, 18; aldormonn II, 9; ọđoro VII, 12 gegen aldur Mt. Prf. 19,3; suundur XX, 7; sunder 
VIII, 6; huidir III, 8; pidir XIII, 36; Juner XII, 29. - Ueber wechsel im suffix der verwandtschaftsnamen s. dort.

V. Im suffix ig bezw. $\bar{i} g,-a g$.

Beide suffixe sind gemeinae. in ig zusammengefallen, doch die spuren einer alten stammabstufung zeigen sich noch im nebeinander von formen mit und ohne umlaut in der wurzelsilbe. So bei monig XII, 6 ; stets in den zusammensetzungeu monigfald Prf. 6,15; XV, 8; monigfaldlice Prf. 7, 3; monigfallice Prf. 7, 18 (s. § 15,1); neben zahlreich belegtem menig, menigo.

Beide formen im gleichen casus finden sich unmittelbar nebeneinander II, 12: nemenigum, unmonigum.

Bemerkung. Das ableitungs- $i$ der schw. vb. II erscheint zu e geschwächt in lytlege III, 30.

VI. Im suffix od, ed bezw. $a d, e d$.

Die alte $e$-stufe mit $i$-umlaut scheint vorzuliegen in hefid(ponna) XIX, 17 (vgl. ahd. haubit) neben gewöhnlichem heafud.

Jüngerer wechsel zeigt sich bei heafut XIII, 9; heafud Mk. XII, 4; uorud XVIII, 3; uearod XVIII, 12; heafod Mt. Prf. 19, 14; V, 18; untudlice II, 2; untodlice Mt. I, 18 rd.; untetlice Prf. 3, 6 (gewöhnlich abgekürzt $u u t^{\prime}$ ).

Suffixablaut liegt wohl auch vor in embihtade XII, 2 gegen ambeht XXI, 23; s. § 19, I.

In den bisher behandelten suffixen zeigt das northumbrische die neigung, an die stelle von ws. $o, e$ wieder die extremvokale $u, i$ treten zu lassen, vgl. auch byrginne XII, 7 .

VII. Die normalgestalt des ws. suffixes nes $(s)$ s. Kluge, NStB. $§ 138$ ist hier nis $(s)$, nis $(s) e$ (s. auch unter konsonantengemination): nes(s)e findet sich nur in cydnese I, 7; cyðnesse, witnese I, 34; ecnese Prf. 7, 7; ondueardnese Prf. 4, 6; smirineses XII, 3; geliorneses II, 1; uitnessa III, 11.

VIII. Das suffix -ung hat vereinzelt die nebenform -ing (Kluge, N.St.B. § 159) in hrining, raecing Prf. 7, 3; racing Prf. 8,11 ; inblawing Prf. 8,6; forecefterfylging Prf. 7, 19; coepinces II, 16 (über die schreibung s. unter gutturalen); rouing $\mathrm{XXI}, 8$.

ong begegnet in ebolsong $\mathrm{X}, 33$; ebolsongas $\mathrm{X}, 36$ (ebolsung Mt. XII, 31; -ung Mt. Mk. L. J. zs. 5; -ong 6); vielleicht in anlehnung an song.

Ws. mastling steht gegenüber moeslen II, 15; s. L. $§ 68,2$. 
§ 25. Vorsilben.

I. $g e, g i$. Die normalform ist $g e$, daneben $g i$ vereinzelt: gibiddes XV,16; giroefa XVIII, 28; gifyllid XIX, 28; gifillid XIX, 28. 30. Von kapitel 20 an aber erscheint $g i$ häufiger, $\mathrm{um}$ in kapitel 21 völlig $\mathrm{zu}$ überwiegen: giarn $\mathrm{XX}, 2$; giseni $\mathrm{XX}, 20$; gicueden XX,24; gifengon XXI, 3 ; giondueardon, gimôetas XXI, 5.6 etc.; s. vorwort u. Skeat's Pref. p. X.

Das $e$ ist abgefallen vor folgendem vokal in to gêcde Prf. 5,13 ; geade IV, 51 ; V, 9 ; gwurnun IV, 51 ; vor folgendem konsonant in neglefad VI, 64; groefa XVIII, 37; greofa XVIII, 33.

ge erscheint als $i$ in isaegde $\mathrm{V}, 15$; als $e$ in neheburas IX, 8.

II. $b e, b i$. Die gewöhnliche form ist be, doch erscheint $b i$ regelmässig in verbindung mit verben der bewegung, wo seine ursprüngliche praepositionelle oder vielmehr adverbielle bedeutung noch gewahrt ist, so in (bieode $\mathrm{Mt}$. $\mathrm{XX}, 30$; bieodon Mk. IX, 30; bifarende L. XVIII, 36; biwarlas L. XI, 42; biwarlde L. X, 31 etc.; s. L. § 73, 2).

Ausserdem überwiegt bi in bituih Prf. 5, 6; 6, 6; IV, 31. 33 (etc.; bi Mt. Mk. L. J. zs. 72; be 12).

Vereinzelt neben be steht $b i$ in bibodo Prf. 7,6; bigeonda XIX, 18 (begienda III, 26); bityndo XX, 19; bityndum XX, 26; bidelfa XIX, 40.

by erscheint in bybyrge XIX, 40; wo wohl das folgende $y$ den schreibfehler veranlasste.

$e$ ist ausgefallen vor vokalischem anlaut in buta I, 3 etc. $e$ ist ausgefallen vor konsonantischem anlaut in blimpe $\mathrm{V}, 14$.

III. fore, for verhalten sich ähnlich $\mathrm{zu}$ einander wie betontes $b \bar{z} \mathrm{zu} b e$; ein nebeneinander beider in demselben wort ist bei J. nicht $\mathrm{zu}$ belegen; über die schreibweise s. L. $\S 73,3$.

IV. af, of. af neben sonst durchaus üblichem of nur in afista II, 17.

$\mathrm{Zu} a$ abgeschwächt erscheint of in adune gestag Prf. 2,1 neben ofdune astag $\mathrm{V}, 4$.

V. oet, ot. Nur at kommt vor, daneben sehr häufig oed s. unter dentalen.

VI. Idg. $\bar{e}$ liegt $\mathrm{zu}$ grunde bei erist $\mathrm{XI}, 24$; erest $\mathrm{XI}, 25$ (=ws. ळerist) neben arisa XX, 9; s. Kluge, PG. I, § 283.

VII. ond, on, un. Beispiele ond: ondfengo VII, 39; ondueardon VII, 46; ondsuare XIX, 9; ondspyrned VI, 61 ; on steht 
für ond in onfoa (vgl. ondfengo) III, 27; daneben ondfoe Mt. Prf. 18, 16; ondfoa Mt. Prf. 21, 5; on: onscecces Prf. 3, 4; ongeattas Prf. 5, 14; onsion XI, 44; onn in onntynde IX, 17 neben ontynde IX, 32;

on vielfach $\mathrm{zu} a$ abgeschwächt: agolden Prf. 1,13; auorden Prf. 3, 11; awarp Prf. 3,12; einmal auch die zwischenstufe 0 : owoerigdon IX, 28; s. S. § 188 anm. 3;

un: ungewammed Prf. 1,6; undoe I, 27; ungeleaffulle Prf. 4,3; für zu erwartendes un steht ond in ondueardnese Prf. 4, 6 (=absentis).

VIII. Für to erscheint einmal te: tehernisse XII, 38.

IX. Für ঠerh begegnet ঠer in ঠertrymmed $\mathrm{X}, 25$.

$\mathrm{X}$. in, inn erscheinen nebeneinander: ingaed Prf. 6, 2; inngaed $\mathrm{X}, 2.9$; s. unter konsonanten-gemination.

$\S 26$. Schlussglieder von compositis.

Infolge von schwachtonigkeit wird ea: 0 : regluordes Prf. 4,6; lecuord Prf. 8, 4; uiðeruorda XIII, 27 (daneben regluard IV, 51; duruard X, 3; dureuêard XVIII, 17); geonduorde XIV, 23 (daneben geonducardon II, 18; genducerde I, 21; geonduardon VII, 52);

ae wird e: môtêrn, sprêcêrn XVIII, 28); S. § 43, 3;

$i$ wird geschwächt zu $e$ in erest V,29(2); XI, 25; ceneht XIX, 11 neben sonstigem erist, aniht.

Andrerseits steht $i$ in geneolicde Prf. 3, 12 neben sonstigem geneolecde, vielleicht in anlehnung an *neolic (ws. néalic).

Schwächung ursprünglicher länge findet sich bei huluco Prf. 7, 12; S. $\S 43,3$ b.

Weitergehende veränderungen sind eingetreten bei hlaferde Prf. 7, 2; XV, 20; hlaford XX, 2; XXI, 12; hlafard XV, 15; hlafdia XX, 16 rd. (<*hlaf-weard,-dige); ebolsong X, 33; eorodmonna XVIII, 12 rd.; ondetend I, $20\left(^{2}\right)$; geondate I, 20; gebiotate Prf. 7, 19; (< ${ }^{*}$ ef-hālsung, *eoh-rād, *ondhātjan, *bihät); hiorode 188,8 (<*hiw-rēd); fultumma 188,6; anlapum XXI,25 (<*fultéam, *ānlēpe); laruu Prf. 2, 6; I, 38 etc. (< ${ }^{*}$ lär-ðёow; doch vgl. Kluge, N.St.B. § 30); uorulde IX, $32\left(<{ }^{*}\right.$ weer-ald);

$\bar{y}$ ist zu $i$ geschwächt in afista II, $17\left({ }^{*} a f-\bar{y} s t\right)$.

Ursprünglich langes $\bar{\imath}$ ist geschwunden in aelc I, 9; II, 10; suelce I, 14; swalce Prf. 2, 3; eghuelc I, 9; hucelc Prf: 5, 13; $i$ in noht Prf. 7,9; tuoelfum Prf. 5,3; s. S. $\S 43$ anm. 4 , 
Kapitel VI. Vokalschwund.

Die auslautsgesetze sind für das north. natürlich dieselben wie für das ws., da ihre geltung ja bereits ins urengl. oder vielmehr wgerm. fällt. Sie werden bei der darstellung der nominalflexion gestreift werden, soweit sie dafür in betracht kommen; im übrigen vgl. S. §§ 130-136; Kaluza $§ 73$.

Im folgenden soll ausser von den synkopierungen nur die rede sein von weiteren veränderungen des wortendes, wenn nämlich infolge des vokalabfalls gewisse konsonantengruppen in den auslaut treten.

$\S 27$. Veränderung des wortendes infolge der wirkung der vokalischen auslautsgesetze.

Tritt muta + liquida oder nasal in den auslaut, so werden die sonorlaute silbisch und entwickeln gewöhnlich einen secundärvokal, dessen qualität sich nach der natur des vokals der vorhergehenden silbe richtet.

I. Regelmässig erscheint sprossvokal vor $r$ und zwar $e$ nach palatalem, o nach gutturalem vokal der vorhergehenden silbe: waeter Prf. 3,10; II, 9; finger XX, 25; undorlice IX, 30; wundar II, 4 rd.; aldormonn II, 9; vgl. Kluge, N.St.B. $\S 30$.

II. Vor $l$ (auch nach dentalen S. $\S 140$ ) erscheint 0 in (feber)adol IV, 52.

III. Silbisches $n$ bleibt nach kurzer silbe meist unverändert, so stets in ðegn I, 43; ðeign I, 45 etc.; $e$ begegnet nur in (monn)- mogen XVIII, 3.

Nach langer silbe ist stets sprossvokal entwickelt und zwar meist dunkler färbung; so in becon Prf. 1, 7; 3,13; II, 18; IV, 54 etc.; tacon Prf. 1, 7; XIX, 19; XX, 25 (becen nur Mt. Prf. 20, 1; XXIX, 30). Der sekundärvokal dringt äuch zuweilen in das innere des wortes ein, und zwar in seiner qualität nach massgabe von S. § 129 verändert, wie in taceno Prf. 2, 1; III, 2; beceno IV, 48; VI, 2.26 etc. (daneben becno II, 23; s. § 28).

IV. Vor silbischem $m$ findet sich $e$ in foedem $\mathrm{I}, 18$; waestem Prf. 2, 6; 6, 15; IV, 36 etc. (Mt. Mk. L. J. zs. 9 gegen woestm 39).

Bemerkung. Eine weitere vokalentwicklung im wortinneren begegnet zwischen $r h$ und $r g$ bei forohtandum Prf. 4, 16; burug $\Pi$, 12; byrig II, 11; vgl. Branne, Ahd. gr. §69a; S. § 213 anm.; Kaluza § 73 anm. 5 ; 
$\S 28$. Synkope von mittelvokalen.

Nach langer wurzelsilbe wird jeder nicht durch position geschützte ursprünglich kurze einzelne mittelvokal synkopiert.

Beispiele sind: I. Vor $l$ : lytle Prf. 7, 14; lytlige Prf. 4,2; gelytlade Prf. 1, 9; deglum Prf. 4, 4; engles XX,12; saule 188, 9 rd.; oeðle IV, 44.

II. Vor $r$ : ođrum Prf. 3, 10; iure Prf. 5, 12; allra Prf. 7, 1; uldre XI, 4; sestras II, 6; suulfre, seulfres 188, 5. 9.

Nach $\S 27$ entwickelter sprossvokal dringt nicht in das innere des wortes, wenn dieses um eine silbe wächst: uintra Prf. 4, 8; VIII, 57; uundra Prf. 8,1; fingre VIII, 6 .

Gegen die regel erscheinen ohne synkope: oðero Prf. 5, 1; II, 1; oðoro VII, 12; moderes III, 4; s. S. § 144 anm. 1.

III. Vor $n$ : Flektierte part. praet. auf -en: gehatne Prf. 1, 14; gewordne Prf. 3, 16; indrungno II, 10; forletno Prf. 7, 17; ferner: drihtnes V, 4; hadnum Prf. 6, 14; âgno X, 3; mérne I, 43.

Die synkope unterbleibt nach $\mathrm{S}$. $\S 144 \mathrm{~b}$ in neteno IV, 12 .

Sprossvokal dringt nicht ins wortinnere bei gebecnas Prf. 3,10; becno II, 23; tacne Prf. 4, 15; uoepnum XVIII, 3.

Gegen die hauptregel unverkürzte formen erscheinen häufig im part. praet., wo besonders grosses schwanken herrscht, vgl. S. § 296 anm. 2; gedolfene Prf. 2, 1; gebundeno XI, 44; geuordeno II, 1 ; ausserdem in aganes Prf. 3, 13 rd.; sprossvokal ist eingedrungen in taceno Prf. 2, 1; III, 2; beceno IV, 48; VI, 2. 26; vgl. § 27. III.

IV. Vor $d$ und $\delta$ : über die praeterita der schw. vb. I vgl. unter stammbildung der schw. vb.

Sonstige belege: heafde XIX, 30; hafde XIX,2; gebegdum

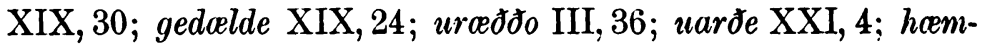
dum Prf. 1, 3.

Formen ohne synkope begegnen in gecerredo Prf. 1, 10; gedoemedo Prf. 5, 9.

V. Vor g: uitga Prf. 3,6; gewitgade Prf. 2,1; suindriga Prf. 2, 5; halge Prf. 3, 7; eadgo Prf. 8, 8; eftgemyndgo II, 22.

Mit erhaltenem mittelvokal erscheint eftgemyndigo II, 17 .

VI. Position schützt gegen die synkope. So erscheinen unverkürzt:

subst. auf -en, -enne: byrgenne Prf. 2, 2; byrgennum V, 28; subst. auf -ere (-are); godspellere Prf. 1,2; uordares IV, 23; 
aber mynetro II, 14 ; mynetra II, 15 ; ferner afterra IV, 54 ; rocgetede 187, 14.

Nach kurzer silbe bleibt der mittelvokal in der regel erhalten, doch herrscht hier vielfaches schwanken; namentlich vor $l$ tritt fast regelmässig synkope ein; s. Kaluza $\S 72$ d. So in miclo VI, 2. 5; micle VII, 37; micla XXI, 11 (micelo V, 3); yflo Prf. 4, 11; III, 20; yfles V, 14; s. S. 296 anm. 2.

Vor $r$ ist ursprünglicher vokal geschwunden bei uatre II, 7; ucettre I, 26.

Vor $n$ findet sich besonderes schwanken wieder in den part. praet. der st. vb.; gecuedne Prf. 1, 13; forcuoedne Prf. 7, 13 (forcuodeno III, 20); regelmässig synkope weist auf: heofnum Prf. 4, 18; heofne III, 13 (nur heofone Mt. XIV, 9); bydno II, 6.

Vor $m$ erscheint synkope bei hlaetmeste VI, 40.

Vor $\delta$ ist der mittelvokal synkopiert in fremðe Prf. 2, 2. 3; sighðana Prf. 1,11.

\section{Zweiter abschnitt: Konsonantismus.}

Kapitel VII. Die halbvokale.

$\S 29 . \quad-w-$

Für die schreibung des halbvokals $u$ stehen das runenzeichen und $u \mathrm{zu}$ gebote, die willkürlich mit einander wechseln; doch findet sich vor oe und $y$ häufiger die rune geschrieben. gegeben.

Bemerkung. Das runenzeichen der hs. wird hier mit $w$ wieder-

uu begegnet in uuritte II,22; fuuluuas I, 25; gegiuudees IV, 10; celaruuas VII, 45; suuord XVIII, 10 ; wu in wualdon VI, 21; uw in at-euwdae XXI, 14; at-euwed XXI, 14.

Wie hier $w u$ für $u u=u$ steht, so ist andrerseits $w u$ in gwurnun IV, 51; wuðuotto VIII, 3 als $u$ u $=\bar{u} \mathrm{zu}$ fassen s. L. $\S 80$. Anlautendes $w$ vor $u$ wird zuweilen nicht bezeichnet, so in ulf X, 12; uldve XI, 4; geunia Prf. 4, 3; geunade XI, 6; unas I, 38; ebenso ist es weggelassen in coed XI, 40; hoed VIII, 25; untearde XVIII, 22.

I. Anlautend erscheint $w 1$ ) vor allen vokalen: waes Prf. 1,5 ; uifes Prf. 1,3 ; woere Prf. 1,6; wunande Prf. 7, 7; uorud XVIII, 3; uêl XVIII, 23; weras VI, 10; uiltu V,6; 
2) in den verbindungen $w r$, wl: aurat Prf. 1, 11; aworat Prf. 1, 11; megulit V, 37;

3 ) in den verbindungen $c w, h w, \delta w, t w, s w:$ gecuedne Prf. 1, 13; ofercuome Prf. 1, 14; huoeðre Prf. 2, 4; Juong I, 27; tucem Prf. 3,8; suiðe Prf. 7, 11.

Bemerkung. Abfall eines anlautenden $w$ findet sich in den mit der negationspartikel ne kontrahierten formen einiger verba: nallað Prf. 4, 16; nyste I, 31; nastu III, 8; noess XI, 15; nere XXI, 23; nutton IV, 22 (daneben nuilt XXI, 18; ui=y? vgl. suindrige); ferner in hu XVII, 22; huu III, 4; noht Prf. 7, 9 (<*nowiht); noeniht I, 3; hlaferde Prf. 7, 2; vgl. anch S. $\S 172$ anm.

II. Inlautend steht $w$ vor allen vokalen ausser $u$ und urengl. $i$ ohne veränderung: stoue Prf. 2,2; auch nach konsonanten: alaruas XI, 47; fuluande Prf. 3,7; vor $u$ und $i$ ist es geschwunden und infolge davon sind öfter kontraktionen eingetreten.

1) Vor $u$ : ealond Prf. 1,11 ( ${ }^{*} a h w \bar{o}>{ }^{*} a h u$ westgerm.); oncneaun XII, 16; gebleoun XX,22; Jrounge Prf. 8,12; doch ist $w$ nicht selten analogisch wiederhergestellt: Jrouung Prf. 7, 19; hrouun VI, 19;

2) Vor $i$ : a I, 17; sa Prf. 4,16; gegevelum XX,12; wohl auch awael' (ed) XX, 1 zu ws. wielwan; smiride IX, 11; smirinise XII, 5;

III. Im silbenauslaut wird $w$ 1) mit vorausgehendem kurzen vokal zum diphthongen verschmolzen: Jea Prf. 5, 16; uintreo $\mathrm{XV}, 1$; treo XV, 2 rd. (oft ganz abgefallen: tre Mt. VII, 18. 19 etc., vgl. Cook); laruu I, 38; larûि XX, 16; das wohl als larū zu lesen ist; vgl. § 26.

2) Nach langen vokalen und diphthongen schwindet $w \mathrm{zu}-$ nächst, wird aber zumeist aus den flektierten formen analogisch wieder eingeführt, namentlich im wortinnern bei synkope eines mittelvokals: stou IV, 20; V, 13; stôu XIV, 3; ठeau XIX, 40 (bei saule 188, 9 rd. steht er im silbenanlaut: zu trennen sa-wle).

$w$ in der stellung nach diphthongen ist gesondert $\mathrm{zu}$ betrachten je nach der entstehung der betr. diphthonge. $w w=$ gemeingerm. $w w$ (got. $g g w$ ), das mit vorausgehendem ursprünglich kurzen vokal zum diphthongen verschmolzen ist, bleibt erhalten in ymbsceaude I, 42 (sceode XX, 11?).

$w w=$ germ. $w$ entstanden durch gemination vor $j$ oder $n$, geht ebenfalls mit vorhergehendem ursprünglich kurzen vokal 
eine diphthongische verbindung ein; erhalten ist es nach dieser in eaunis Prf. 1, 1; aeteaude Prf. 1,5. etc.; <*aujan < ${ }^{*}$ agujan (zu éage vgl. Hirt, Beitr. XXII, 231; vgl. auch Paul, Beitr. VI, 97).

Die lautgebung dieses verbums ist schwer zu beurteilen, ein *aujan könnte für das north. nur êwan (ev. égan, vgl. cégan) geben, das dem ws. iewan entsprechen würde; die offenbar umlautlose bildung (vgl. dagegen Paul, Beitr. VI, 97) lässt sich vielleicht auf doppelformen nach der II. schw. konj. zurückführen; dafür spricht, dass aed-eauade bei Mt. Mk. L. $8 \mathrm{mal}$ belegt ist neben dem ebenfalls umlautlosen praeteritum ot-eaude s. oben. Die ausserdem noch vorkommenden formen oet-euwdoe $\mathrm{XXI}, 14$; aed-eude V, 20 (über die schreibung s. oben und $\S 20$, VIII) entsprächen dann ws. eowde S. $§ 408$ anm. 10; 73 anm. 1 aus *awida.

Geschwunden ist $w$ in $\partial r e a \delta$ VIII, 46 ; und onsion XI, 44; onsione VII, 24 (< ${ }^{*}$ segwoni Kluge, N.St.B. § 229); sowie séado XII, 6; XIII, 29, vgl. $§ 13$ (got. siujan).

Endlich findet sich $w$ durch vokalsynkope im silbenauslaut in der gruppe éow, die aus ursprünglich *awi entwickelt worden ist, vgl. S. § 73 anm. 1; es ist erhalten in ceoulas VI, 13; geschwunden dagegen zugleich mit dem von ihm entwickelten dunklen vokal in ede Prf. 6, 4; s. Bülbring, E. St. XXVII, 85 fussnote 2. Ueber die gruppen eow, iow und ihre schreibung $\mathrm{s}$. $\S 20,8$.

$$
\S 30 .-j-
$$

I. Anlautendes $j$ wird mit $i$ nur in fremden eigennamen bezeichnet: iohannem 187, 7; iacobe IV, 12; iordanen I, 28 etc.; sonst wird stets $g$ geschrieben: gie Prf. 4,16; gif Prf. 5, 14; begonda I, 28; geres XI, 49; gee IV, 35; gêe XIV, I9; giungra XXI, 18 etc., vgl. auch $\S 21$, I.

II. $j$ ist inlautend stets geschwunden nach langer geschlossener silbe - beispiele in der flexion der schw. verba nach I sowie der jo; $\{\bar{a}$-stämme - ebenso fast regelmässig zwischen vokalen, so in freond XI, 11; gefriad Prf. 5,15 ; eade $<*$ iijiade; ece $<{ }^{*}$ ejece etc.; nur vereinzelt begegnet $j$ nach langem vokal bezw. diphthong im verbum ceiga Prf. 1, 3; ferner in floege VI, 22; vgl. § 17, IV; auslautend in heig VI, 10. Als $j$ hat wohl auch das $g$ in trugude II, 24; fulguge Prf. 4, 1, dem ws. $w$ entspricht, zu gelten; vgl. auch Braune, Ahd. gr. § 110 anm. 3. 
Erhalten ist inlautendes $j$ auch bisweilen nach kurzem vokal + konsonant, d. h. eigentlich nur nach kurzem vokal $+r$ (hergas Mt. XX, 7; hergum Mt. Prf. 6, 19); doch ist nach diesem muster auch bei anderen konsonanten häufig die gemination wieder beseitigt worden und $j$-bildung dafür eingetreten: getrymies XV, 27; S. $§ 400$ anm. 2.

Ueber die bildungssilbe $i g$ der schw. verba II s. S. $§ 175$ anm.

Kapitel VIII. Die liquidae und nasale.

$\S 31 . \quad-r$.

I. $r$ ist häufig in allen stellungen belegt: rodes Prf. 8,12 ; brydlopum Prf. 1, 3; ward Prf. 2, 2; uoerc X, 33; mara Prf. 7, 2 etc.; seltener verdoppelt: gecerredo Prf. 1, 10; lehnwort carr I,42.

II. Inlautendes $r$ erfährt oft metathese: 1) Vorvokalisches $r$ tritt gern hinter den vokal, wenn diesem $n n$ - oder $s$-verbindungen folgen: gearn Prf. 6, 12: iornendum Prf. 8, 3; bearnende V, 35; bernad XV, 6; burna XVIII, 1; goers VI, 10. - Vor $n n$ <zn: (môt-, sprêc-) êrn XVIII, 28; ferner in đirde Prf. 8, 10.11 (Jridda XXI, 14); über forma Prf. 1, 7 und fruma Prf. 1, 13; s. S. $§ 179,1$.

2) Der umgekehrte fall tritt ein vor ht: gebrehtnige Prf. 6,17 (berhtnade XII, 28).

III. $r$ entspricht einem germ. $z$ in gecoren Prf. 1,2 mit gr. wechsel zu céosan; mara Prf. 7, 2; earlipprica XVIII, 10; $r d$ einem $z d$ in reordana Prf. 7,$10 ;$ mearda $\mathrm{IV}, 36 ; r r$ einem $r z$ in arro XV, 18.

IV. $r$ ist ausgefallen in endebrednisse Prf. 2,4; und häufig in dem komparativ aldra: celdum Prf. 5, 7; 6, 13; celdo Prf. 6, 1; s. L. $\S 86 ; s r>s s$ vereinfacht $\mathrm{zu} s$ in wyrse V, 14 (got. waírsiza). flexion.

Ueber $r$ in faestern Prf. 5, 8; uoestern Prf. 3, 5 s. unter subst.

$$
\S 32 . \quad-l-.
$$

$l$ erscheint in allen stellen des wortes; oft auch geminiert: lust Prf. 1, 3; tuufald Prf. 1, 3; foloe XII, 15; godspell Prf. 1, 4. 5; alle Prf. 1,10; 2,3; sealla XIII, 11; s. auch unter gemination. $s l$ wird assimiliert zu $l l$ in oullico IV, 23.

Nach unbetonter silbe wird $s l \mathrm{zu} l s$ in der ableitung -els bei receles XIX, 39; milce XI, 43 ist verschrieben für micle. 


$$
\S 33 .-m-
$$

$m$ bezeichnet den labialen nasal und begegnet häufig in allen stellungen: meigð Prf.1,11; homdım Prf.1,3; smolt XX,19; geminiert in ungewaemmed Prf. 1,6.

$m$ fällt aus vor den tonlosen reibelauten $f, b, s$ unter längung des vorausgehenden vokals; belegt nur: fif Prf. 4,14; fiftenum XI, 18; fiftig VIII, 57.

\section{§ 34. $-n$ -}

$n$ hat die geltung des dentalen nasals in nacod XXI, 7; an Prf. 1,2; insiht Prf. 1, 1; onginned Prf. 1, 1; den gutturalen nasal bezeichnet es in gesungen Prf. 2, 2; Juong I, 27.

$n$ fällt aus vor den stimmlosen reibelauten $f, b, s$ (vor $h$ schon urgerm.) unter längung des vorausgehenden vokals: cyðnisse Prf. 1,4; cuðlice Prf. 3,11 ; cuðe I, 31; suide Prf. 7,11; muðe XIX, 29; soðlice Prf. 2, 3; oðrum Prf. 3, 10; uuðuotto VIII, 3; oð XXI, 22; uusc XIII, 33; îs Prf. 2, 5; usih I, 14; gebrohte Prf. 8, 5; geoehtadon V, 16.

Die ausstossung des nasals findet nicht statt, wenn derselbe erst durch synkope eines vokals unmittelbar vor den reibelaut getreten ist, so in cloensunge II, 6; vgl. ahd. kleinisōn.

Auslautendes flexivisches $n$ wird unterdrückt im infinitiv, plural des optativs praeteriti (aber nicht des indikativs) und in der schw. deklination: gemana Prf. 1,3; lichoma Prf. 3,13 etc.; s. unter formenlehre.

Ebenso fällt $n$ ab in den adverbien auf an: befora Prf. 3,3 ; before Prf. 3, 5; ufa Prf. 3, 7; 4,2; buta Prf. 3, 14; ठona Prf. 4, 11; begeonda I, 28; huona I, 48; soðða Vl, 16; binna XVIII, 15; XX, 26; ûte -bûte XVIII, 16 (nur einmal neaðan VIII, 23; ûtan 188, 4 .

$n$ fällt fast stets aus in cyning zwischen $i$ und $g$ : cynig I, 49; VII, 41; XII, 13. 15; XVIII, 33. 37 (2) etc.; cyninge Prf. 4,15; einmal auch penicas XII, 6 gegen sonstiges penning; sn wird assimiliert $\mathrm{zu} n n$ in Jionne $\mathrm{V}, 6$.

Die praeposition on wird in der komposition gern zu $a$ verkürzt, s. $\S 25,7$. 
Kapitel IX. Labiale.

$\S 35$. $-p$ -

$p$ erscheint anlautend in pol V,2.4. 7; ferner in folgenden lehnworten: pund XII, 3 ; penninga VI, 7; plett X, 1; palmana $\mathrm{XII}, 13$; palmtreo XV, 4 ; palmung $\mathrm{XV}, 2$; pytt IV, 11 ;

in- und auslautend fiudet es sich auch in germanischen wörtern öfter: hwisprendo Prf. 4, 20; sprecco VIII, 26; blimpe V, 14; woepnum XVIII, 3; uоepađ XVI, 20; scîpo X, 3; suuopa II, 15; lehnworte: copp XIX, 29; apostol XIII, 16;

geminiertes $p$ begegnet in upp Prf.5,13; earlipprica XVIII,10;

$b p$ erscheint im lehnworte purbple XIX, 5 ;

$b$ findet sich für $p$ in biscob 188, 1 ; biscobe, biscobas XVIII, 24. 26; biscobi XVIII, 22, wofür wohl die romanische aussprache verantwortlich $\mathrm{zu}$ machen ist.

$$
\S 36 . \quad-b-(=\text { urgerm. } \delta) \text {. }
$$

$b(<$ germ. $\delta)$ bezeichnet den stimmhaften labiodentalen verschlusslaut und hat in dieser geltung seine stätte im anlaut, inlautend in der gemination und in der verbindung $m b$. bebead Prf. 1, 4; brydlopum Prf. 1, 3; blodum I, 13; birilum II, こ; nabbad II, 3; habbas X, 10; auch vereinfacht: habað Prf. 7,17; XVI, 22; lomb Prf. 3,6; ymb (cerde) Prf. 3, 11.

Als gleitlaut zwischen $m$ und $r l$ ist $b$ entwickelt in getimbred II, 20; symble VII, 6 (lat. simul, semel).

Vereinzelt fällt $b$ ab in $y m$ Prf. 3,$13 ;$ S. $\S 190$ anm.

In allen anderen fällen hat germ. $t$ den lautwert des labialen stimmhaften reibelautes bewahrt und wird in der schrift meist durch $f$ wiedergegeben; so: uifes Prf. 1, 3; efne Prf. 1, 7; of Prf. 1,2; ofer Prf. 1, 14; stafum V, 47 etc.

Im auslaut mag wohl schon stimmlosigkeit eingetreten sein, so in fordraf II, 15.

Nur selten findet sich in dieser geltung die schreibung $b$ : ebolsung $\mathrm{X}, 33$; ebolsungas $\mathrm{X}, 36$; in den lehnworten: diubul Prf. 5, 3; feber IV, 52.

$$
\S 37 .-f-\text {. }
$$

$f=$ germ. $f$ bezeichnet den stimmlosen labiodentalen reibelaut und kommt an- und inlautend häufig vor: foerde VIII, 1; fylgendo Prf. 3, 8. 
DIE SPRACHE DER NORTHUMBR. INTERLINEARVERSION. 51

Inlautend ist $f$ vermutlich stimmhaft geworden, so in ahefen XII, 32; hefignise XVI, 21.

Durch folgendes $j$ geminiertes $f$ ergiebtt $b b$ : hebbendum XI, 41 ; auch vereinfacht: underhebendum XVII, 1 .

$f$ erscheint in fremden eigennamen für lat. ph: caifa, caifas XVIII, 13. 14.

\section{Kapitel X. Dentale. \\ $\S 38 . \quad-t$.}

Der dentale stimmlose verschlusslaut $t$ ist in allen stellungen häufig zu belegen: to Prf. 1, 4; tuoelf XI, 9; treo XV, 2 rd.; gehatne Prf. 1, 14; awrat Prf. 1, 11; breost XIII, 25.

Geminiertes $t$ erscheint in sittendo II, 14; sette XV, 13; vom schreiber verdoppeltes in: gesetted Prf. 2, 2; wriottana Prf. 2, 4 (s. unter gemination).

$d$ wechselt mit $t$ in oed-: adeaude Prf. 1,8 (ced-in diesem verbum bei Mt. Mk. L. J. zs. 80 ; at- 13; atd- 4; daneben einmal œð XVIII, 13; während sonst die praeposition stets $œ t$ lautet).

Neben 8 ad- in adeaunisse steht einmal aet- ateaunis Prf. 1,1 ; neben 10 red- in aedgeadre stehen 4 cet- IV, 36; Mt. Prf. 1,6; Mk. II, 15; L. VII, 49; ferner finden sich 86 hucet neben 114 huced; ausserdem 18 hucetd; 1 hucedt; 1 hucedd.

Für unrotnis begegnet unrodtnise XVI, 20; vgl. L. § 95.

Für ws. $t l<b l$ erscheint $d l$ in spadl IX, 6; hehsedle XIX, 13; bydle IX, 31; s. S. § 196, 2; vgl. auch $§ 40$.

Ueber $t$ in den verbindungen $f t$, st, $h t \mathrm{~s}$. $\S 44$.

$$
\S 39 . \quad \cdot d-
$$

$d$ bezeichnet den dentalen stimmhaften verschlusslaut und erscheint in allen stellungen: doeg Prf. 4,19; drihten Prf. 1,9; moder, rode Prf. 1, 4; brydlopum Prf. 1, 3; foerde Prf. 1, 4; gedroefedo Prf. 6, 1; geminiert in biddo XVII, 9 (2). 15. 20; vom schreiber verdoppelt bei goddes Prf. 3, 4; gloedde XVI, 20; Jidder VI, 21; s. unter gemination.

$l d=$ got. $l d$ erscheint in hehstald Prf. 1,$2 ;$ agolden Prf. $1,13$; über $l d=$ got. $l]$ s. $\S 40$.

$l d l$ erscheint als $l l$ in tuifallice Prf. 7, 10; monigfallice Prf. 7,$18 ; \mathrm{X}, 10$.

$d$ nach stimmlosen lauten wird $\mathrm{zu} t$ in den praeterita der schw. verba I (s. dort): gesette Prf. 1, 7; gemoette I, 41; geondete 
Prf. 3,6; geondate I, 20; doch bleibt es vor stimmlosem laut in bloedsade VIII, 48 (nur einmal bledtsigas XIII, 13; vgl. § 23, VIII; ausgefallen ist es in milsa 188,$11 ;$ S. $\S 198,4$ anm.

Ausserdem erscheint $t$ fälschlich öfter für $d$ in hehstaltnisse Prf. 1,3; untetlice Prf. 3,6; heafut XIII, 9; heartlice XII, 32; im praet. und part. praet. der schw. verba: tuiaton XIII, 22; ingemoetet Prf. 2,3; inlichtet Prf. 6,2; geinsetet Prf. 1,10; gesettet Prf. 2, 2; gededet XII, 33; gebiotate Prf. 7, 19.

$\delta$ ist für $d$ geschrieben in Jegla Prf. 8,5; derne-legerscipe VIII, 3; cuoeðon XVIII, 7; aedgceðre XX, 4; in fremden eigennamen iuðeos XVIII, 38; iuðeum XIX, 40; iuðeana XIX, 42; magðalenisca $\mathrm{XX}, 1$.

$d(d)$ für romanisch $d$ erscheint in lcedinisc XIX, 20; loeddin XIX, 20; daneben latin XX, 24 rd.

$d+t$ wird vereinfacht $\mathrm{zu} t$ in hunteantig XIX, 39; XXI, 11 . Nach konsonant $+d, t$ geht $d$ verloren: sende I, 32; geoehton $\mathrm{XV}, 20$; ebenso bisweilen im proklitischen -ond, vgl. § 25 , VII.

$d$ steht in grammatischem wechsel mit $\delta, \mathrm{s}$. dort.

$$
\S 40 . \quad-\delta-
$$

Der dentale reibelaut wird gewöhnlich mit $\delta$ bezeichnet, das in allen stellungen erscheint: đeignum Prf. 1, 1; ðe Prf. 1,2; broðer I, 40; cyðnisse Prf. I, 4; meigð Prf. 1, 11; warð Prf. 2, 2; onginneð Prf. 1, 1.

In fremden eigennamen wird zuweilen $\delta$ für th geschrieben: beðsaida I, 44; beðania XI, 1; arimaðia XIX, 38; marða XI, 20 -(daneben martha XI, 1. 19 u. ö.); đomas XIV, 5; scarioð XIV, 22 (= scariotis).

Dagegen wird $p$ nur angewandt in den abkürzungen $\bar{p}=$ ðxt; $\not t e=\delta c t t e$ (nur dreimal ausgeschrieben $\delta a t t e$ Prf. 7, 1; II, 10; IV, 34); oд $=$ oððcet XXI, 22; ausserdem vereinzelt $p a c$ cille $\mathrm{V}, 35 ;$ pidir XIII, 36 ; pone X, 12; XI, 39 .

th für $\partial$ ist geschrieben in throuung Prf. 7, 19 .

Geminiert erscheint $\delta$ in soðða VI, 16; оðða XVIII, 34.

Altes $l p$ und nach langem vokal altes $p l$ gehen in $l d$ bezw. $d l$ über: uuldor I, 14; ad(o)l IV, 52; adligne XI, 1 ; gewidloed XVIII, 28; spadl IX, 6; in diesem wort wie auch in den kurzvokaligen hehsedle XIX,13; bydla IX, 31, die mit suffix -tlo gebildet sind, erscheint im ws. $t l$; vgl. $§ 38$; s. Kluge, N.St.B. $\S \S 97.142$. 
$t+\delta$ wird $\mathrm{zu} t t$, das nach konsonanten vereinfacht wird: nastu III, 8; XIX, 10; uiltu V, 6; бotte Prf. 7, 1; II, 10; IV, 34.

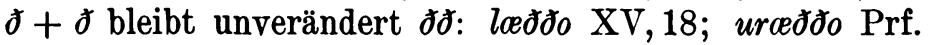

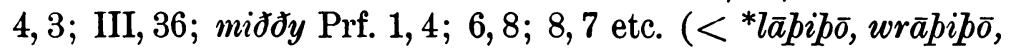
mið $+\delta y$ gegenüber ws. mitty aus mid $+\not y y)$.

$s+\delta$ wird st: cuoeðestu I, 22; hafdlestu XIX, 11; lufastu XXI, 16. 17; lufoestu XXI, 15; ondsucerestu XVIII, 22; uoepestu $\mathrm{XX}, 15$.

$\delta$ gegenüber ws. $d$ erscheint durchweg bei fremðe Prf. 2, 2. 3; X, 5; hraeðe XI, 29; hrôøe XIII, 27; roeðe XX, 15; hraður $\mathrm{XX}, 4$; hundrað Prf. 8, 9 (inlautend $2 d$ : hundrades Mt. Prf. 19, 19; hundradum Mk. VI, 21 rd.); mið I, 1.2 (etc., s. auch oben miððy); gedeðet XII, 33 (gedeðed Mk. VII, 10; L. XXIII, 22).

Ein wechsel zwischen $\partial$ und $d$ findet statt in hoedno XII, 35; XII, 20; høedna VII, 35; hoednum Prf. 6, 14 (hoeðen Mk. VII, 26; haðin L. XVII, 16; haððinra L. XXI, 25 etc.).

Ausserdem ist $d$ fälschlich für $\delta$ geschrieben in broder Prf. 3, 9; haeled Prf. 4, 9; odro Prf. 5, 18; cuoedo XIV, 2; XVI, 26; cuoedendo Prf. 4, 5; gecuoed Prf. 4.8 (mit $d$ aus dem plural, vgl. me. quod).

$t$ begegnet öfter für $\delta$ in inting XVIII, 38; XIX, 6; intîng XIX, 4 (inđing L. XXIII, 14. 22).

$\delta$ steht in grammatischem wechsel mit $d$, s. dort.

$$
\S 41 . \quad-s-
$$

$s$ erscheint überaus häufig in allen stellungen: soðXVI, 5; insiht Prf. 1, 1; unascended Prf. 1, 12; gescyrte III, 2; sprace Prf. 4, 4; gestag Prf. 2, 1; fisces VI, 9; lust Prf. 1, 3.

$s n$ wird assimiliert $\mathrm{zu} n n$ in Jionne $\mathrm{V}, 6$.

$r s$ wird assimiliert zu $r r$ in uurresta II, 10; uirresta II, $10 \mathrm{rd}$.

$s$ steht in grammatischem wechsel mit $r$, s. dort.

Die affrikata $t s$ wird mit $z$ und $t z$ bezeichnet und erscheint in dem fremden eigennamen nazareth I, 46; nazarenesca XVIII, 7; natzarenisca XVIII, 5; XIX, 19.

Kapitel XI. Velare und palatale.

$$
\S 42 . \quad-c \text {. }
$$

$c$ ist das zeichen für den velaren und palatalen stimmlosen verschlusslaut (vgl. S. §§ 206.207) und ist in jeder stellung 
häufig zu belegen: cald XVIII, 18; cesto XIII, 29; costum Prf. 7,10; culfrae I, 32; cyninge Prf. 4,15; clioppendes Prf. 3, 5; cnceht VI, 9; cuoellanne V, 18; eft-geceigde Prf. 1, 3; gecerredo Prf. 1,10; folce Prf. 6, 10; uoerc IX, 3; sciu XIX, 32. 33; fisc XXI, 9. 13.

Anlautend im fremden eigennamen: cregisc XIX, 20.

Geminiert erscheint $c$ in waccennes Prf. 3,14; vom schreibel verdoppelt in gebrucce VI, 5 ; s. unter gemination.

Auslautendes $c$ geht öfter in $h$ über, so in ah Prf. 3, 5. 15 etc. (ac nur Mt. XVI, 23; L. Prf. 6, 18); iuih, iuh, iwh Prf. 5, 15; I, 26; IV, 25 etc. (iuc nur III, 7); usih I, 14 (daneben häufiger usic); meh XIII, 21 (daneben häufiger mec); ðeh XVII, 5 (daneben häufiger $\partial e c)$.

Daneben begegnen auch die schreibungen $c h$ und $g$ in mech VI, 35; XIII, 8; đech VIII, 11; iuch I, 15; usig Prf. 6, 13; I, 22.

Vor $t$ geht $c$ in $h$ über in lehtun XVIII, 1; lehtune XVIII, 26.

In der verbindung $n c$ findet sich für $c$ häufig die schreibung g oder cg, gc: dringað Prf. 5, 7; indrungno II, 10; scryngcara $V, 3$ (drincga L. XXII, 30).

Hier liegt vermutlich eine erweichung der fortis zur lenis, vielleicht gar eine neigung zur palatalen affrikata vor.

Palatalisierung wird offenbar auch bezeichnet durch die schreibung ( $g) c g$ für $c$ nach kurzem vokal: brecg XVIII, 6; bagcgling XX, 14; gebracged XIX, 36; cg für cc in laecga XII, 35; stycg XIX, 23; vgl. Bülbring, Anglia, Beibl. IX, 75. 76.

\section{$\S 43 . \quad-g$ -}

$g$ begegnet häufig in allen stellungen; über seme lautwerte vgl. S. §§ 206; 211-216; godspellere Prf. 1, 2; gaste Prf. 3, 7; gaers VI, 10; gyrdeð XXI, 18; gefe XVI, 2; gingra XXI, 18; gleed III, 29; ðeignum Prf. 1, 2; gesagd Prf. 3, 3; eft-geceigde Prf. 1,3; argscipe Prf. 5, 8.

Geminiertes $g$ geschrieben $c g, g c, g c g$ erscheint in $s t y c g$ XIX, 23; licgende V, 6; forhygcanne Prf. 7, 12; sagces XVIII, 29; sagcganne XVI,12; saegcgo XVI,25. Zur schreibung vgl. Hempl, Angl. XXII, 375-383.

Vom schreiber verdoppeltes $g$ begegnet in tuiggo $\mathrm{XV}, 5$; tuigga XV,6; tuiggum Prf. 6,12 ; s. unter gemination.

$\mathrm{Zu}$ den schreibungen tuicgo XII, 13 (rocgetede 187, 14; $\mathrm{s}$. Bülbring a.a.o. p. 76); vgl. § 42 letzter absatz.

Für (palatales) $g$ nach $r, l$ wird öfters $i g$ geschrieben, wenn 
$y$, i, e vorhergeht: byrig Prf. 2, 1; I, 28; in geberigde II, 9; owoerigdon IX, 28; fylig XXI, 22.

Auslautendes $g$ wird nach langem palatalen vokal $\mathrm{zu} h$ nur in hehstald Prf. 1, 2; S. $§ 214$ anm. 1 (unter einfluss des stimmlosen st?); nach kurzem vokal in unbetonter silbe bei Jritðeih Prf. 4,8; Jrittih V, 5; fftih Prf. 8, 9.

Die vermittelnde schreibung $g h$ begegnet in meghø I, 43; anigh V, 19; S. § 214 anm. 5.

Dagegen findet sich der übergang zu $h$ nach langem velaren vokal oder $r, l$ nicht.

In geðuoh Prf. 7, 1 liegt wohl altes $h$ vor, doch vgl. S. § 392, 2.

In der verbindung $n g$ findet sich für $g$ zuweilen $c$ und $c g$, gc geschrieben, so in coepinces II, 16; penicas XII 6 mit ausfall des $n$, hyncgreð VI, 35; fingcer $\mathrm{XX}, 27$; vgl. § 42.

Zwischen konsonanten ist $g$ ausgefallen in merne $\mathrm{I}, 43$; nach palatalem vokal vor $n$ in gihrîno 188,4 .

Auslautendes $g$ fehlt in sunnedoe V, 18.

\section{$\S 44 . \quad-h$ -}

$h$ steht anlautend vor allen vokalen; ferner in den verbindungen $h l, h r, h n, h w$. Seine artikulation als hauchlaut war offenbar sehr schwach; denn es findet sich vor obigen konsonanten (seltener auch vor vokalen), wo es etymologisch nicht berechtigt ist; andererseits ist altes $h$ vor $l, c, n$ vielfach weggelassen worden.

Etymologisch unberechtigtes $h$ findet sich:

1) vor $l$ : hlif Prf. 4, 18; hloetmesta VI, 39; VII, 37 (unter den 32 belegen bei Mt. Mk. L. J. nur 7 mit richtiger schreibung, darunter loetmeste XVI, 23;

2) vor $r$ : gihrîno 188,4; hrîpes IV, 35; hrioppađ IV, 36; hriordað XXI, 12; gihriordadon XXI, 15; hrouun VI, 19;

3) vor vokal: hage XIX, 24; hagen XIX, 27; hoelc III, 21; hearnunge Prf. 7, 10.

Für $h w$ begegnet $w h$ in foruhon XII, 5 .

$h$ ist weggelassen vor $l$ : lâdanne IV, 7 ; gelionade XIII, 12; laf XIII, 26; XXI, 13; lâf XIII, 26; XXI, 9; vor $r$ : raeðe XX, 15; rining Prf. 8,7.

Im ursprünglichen anlaut ist $h$ geschwunden bei nabbað II, 3 ; noefge IX, 8.

Inlautendes einfaches $h$ und $h w$ vor vokalen schwindet: 
ealond Prf. 1, 11 (mit kontraktion); onfoas Prf. 3, 3; scoes I, 27; geseað I, 39 (mit kontraktion); gesiið Prf. 5,17; 7,5; S. §§ 222,1; 166, 6; fleas X, 5; geðoas XIII, 6; tuiaton XIII, 22; sweor XVIII, 13; fiftenum XI, 18.

$h$ schwindet im zweiten gliede einiger alter composita: geondete Prf. 3, 6; anlapum XXI, 25.

Geminiertes $h$ begegnet in toherende XI, 35, wo es aber mit einfachem $h$ wiedergegeben wird; vgl. S. $\S \S 220$ anm. 1; 222 anm. 4.

Die alten verbindungen $h t$ und $h s$ bleiben erhalten: insiht Prf. 1, 1; geworhte Prf. 1,8; daneben die schreibungen cht, ct: inlichtet Prf. 6,2; inlichtade Prf. 6, 1; getractad IX,7; gh: anight $\mathrm{V}, 30$; IX, 33.

Für altes $h s$ wird $x$ geschrieben: exin II, 14; auexe III, 30 lixende $\mathrm{V}, 35$; foex $\mathrm{XI}, 2$.

$h$ erhält sich, wenn durch vokalsynkope ein stimmloser konsonant dahintertritt: geschr. gh in sighðana Prf. 1,11.

Dagegen schwindet $h$ zwischen vokal und stimmhaftem konsonanten: hêra IV, 12, s. auch S. $\S 166,6$; hel XIII, 18; gesêne Prf. 3, 11; bituien IV, 33; heannissum VIII, 23 mit verdoppelung des $n$ nach S. $\S 222$ anm. 2 ; auch in den compositis geneolicde Prf. 3, 12; eorod XVIII, 12 rd. Dagegen ist $h$ einmal vor $l$ erhalten in Juahles XII, 3; S. § 222 anm. 4.

$h$ ist ferner geschwunden vor $s$ in wastem Prf. 2,6 ; sestre II, 6 ; sesta IV, 6 .

Zwischen $r$ und $h$ ist $e$ eingeschoben in geberehtnad XIII,31; $\mathrm{XV}, 8$; vgl. byrig.

$h$ im auslaut bleibt erhalten: ahoh XIX, 6 ; unruh XIX, 23; feh 188,5; daneben kommen die schreibungen $c h$ und $g h$ vor: Jaech IV, 2; gesagh VII, 52.

Kapitel XII. Allgemeines über die konsonanten.

§ 45. Gemination.

Germanische verdoppelung findet sich bei $l l$ : godspell Prf. 1,4; scillinga XII, 5; full I, 14; ucelle Prf. 4,4; spillo VI, 39; bolla XIX, 29;

bei rr: gecerredo Prf. 1, 10; fearr XXI, 8; lw. carr I, 42;

bei $m m$ : ungewammed Prf. 1,6; 
bei nn: onginneð Prf. 1, 1; winnes Prf. 2, 6; monno Prf 4, 7; sunnedoeg Prf. 4, 9 ; penninga VI, 7 ; binna XVIII, 15; acennes XVI, 21;

bei ઇð: оðða XVIII, 34; s. S. § 226.

bei $c c$ : stycg XIX, 23; vgl. $\S 42$ letzter abs.

Westgermanische gemination vor $j$ begegnet in getryccad XVI, 33; wceccennes Prf. 3,14 ; hopbbende V, 5; middangeardes Prf. 3,7 ; gebiddas Prf. 7, 11; forhygcanne Prf. 7, 12; bebycgendo II, 14 ; licgende V, 6 ; scegcganne XVI, 12 ; saegcgo XVI, 25; vgl. $\S 43$; cuoellanne V, 18; uillo I, 13; synnum Prf. 1,7; cynn XI,48; sittendo II, 14. Lehnwort: pytt IV, 11.

$f j>b b$ : hebbendum XI, 41 .

Gemination durch zusammenrücken zweier ursprünglich getrennter konsonanten zeigt sich bei ungeleaffulle, urœеððo Prf. 4, 3; loeðठo XV, 18. 25; rummôd Prf. 7,7.

Doppelschreibung einfacher konsonanten begegnet nach kurzem vokal bei onscecces Prf. 3,4; locc Prf. 6, 2; spreccende I, 37; accenned III, 3; baccling VI, 66; reccone XVIII, 27; acced XIX, 29; tobroccen XXI, 11; Jceccille Prf. 4, 12; goddes Prf. 3, 4; hidder IV, 15; Jidder VI, 21; gloedde XVI, 20; becedd XIX, 12; loeddin XIX, 20; tuiggum Prf. 6, 12; illca Prf. 6, 8; Jrcell XV, 15 (an. = Jrcell); frumma Prf. 1, 12, cymmende I, 9; cymm XI, 48; nomma XVII, 12; genimme XVII, 15; fultumma 188,6; huonne I, 48; gesuanng XIX, 1; linninum XIX, 40; clioppendes Prf. 3, 5; gegrioppanne Prf. 5, 5; hrippe IV, 35; scipp VI, 17. 19; upp $\mathrm{XI}, 41$; ucess IX, 25; gesettet Prf. 2, 2; wriottana Prf. 2, 4; ongeattas Prf. 5, 14; gotteno Prf. 6, 11; uittnesse I, 7; uattre I, 26; fatto II, 6 ; ett IV, 31; nutton IV, 22; toslittnisse VII, 43; geflitt IX, 16; hlott XIX, 24; satt XIX, 13; lyttel VI, 22; snyttro 188,11; vgl auch S. § 228;

nach langem vokal bei gebrucce VI, 5 ; taccon XIX, 20; cuommon XVIII, 20; hleatte XIX, 24; lettes XI, 44.

Vereinfachung von gemination findet sich selten im wortauslaut, regelmässig nur beim suffix -nis $(<(n)$ assus, $(n) i s s u s)$, ateaunis Prf. 1,1 ; dagegen nett XXI, 6 ; gedd X, 6 ; cynn XI, 48; sibb Prf. 8,5; daneben sib Prf. 7,8; aldormonn II, 9; daneben aldormen II, 8 .

Häufiger wird geminata vereinfacht im silbenauslaut im wortinnern: unsibsumnise Prf. 6,1 ; syngade Prf. 5, 10; ymbcerde Prf.3,11; gefylde Prf. 4, 15 neben fyllnisse I, 16; synngega VIII,11. 
Vereinfachung tritt stets ein nach $r$, das metathese erfahren hat: gearn Prf. 6,12; iornendum Prf. 8, 3; bearnende V, 35; burna XVIII, 1; Jirde Prf. 8, 10.

Geminata nach unbetonter silbe wird vereinfacht: rocgetede 187, 14.

Ausserdem begegnet einfache schreibung alter geminata bei adeaunise Prf. 2,1; degelnise Prf. 3, 14 neben cyðnisse Prf. 1,4 etc.; rumode Prf. 7, 13 neben rummôd Prf. 7, 7; đises II, 10 neben Jisses XI, 9; ucle IX, 7 neben ucella IV, 12; habad Prf. 7, 17 neben habbad VI, 53; selo IV, 14 neben sello IV, 14; niomane Prf. 8, 2 neben gesaeccanne Prf. 8, 4; gecened I, 13 neben acenned Prf. 3, 4 .

\section{$\S 46$. Die gruppen $f t, h t$, st.}

Alle labiale bezw. velare $+t$ werden zu ft bezw. ht: frumsceaft Prf. 1,12; insiht Prf. 1, 1; geworhte Prf. 1,8; bebohton II, 16;

$\mathrm{zu} h t$ in gecnyht XI, 44; s. S. $\S 407,2$;

dental $+t$ ist zu st geworden in nast III, 10; fostra $\delta$ VI, 31 .

$\S$ 47. Grammatischer wechsel.

Wechsel von $s$ und $r$ : gecêas XIII, 18; gecoren Prf. 1, 2.

Wechsel von $\delta$ und $d$ : geucerठ I, 17; geworden Prf. 1,6; gecuceð XVIII, 21; cuoedon I, 22; vielleicht hierher auch fremðe Prf. 2, 2; mið Prf. 3, 2 gegen ws. fremde, mid.

Wechsel von $h$ und $g$ : slaes XVIII, 23 (<*slahis); monnslaga Prf. 5, 16; onfoa III, 27 (< *fanhan); feng Prf. 8, 9; Juahles XII, 3; geðuogun Mk. VII, 3; teigða I, 39 gegen ws. tēoða (<*tehonpa); sighðana Prf. 1,11; gesegon Prf. 8, 8 (ws. sawon).

\section{Teil II. Flexionslehre.}

\section{A. Verbalflexion.}

Kapitel XIII. Die endungen.

$\S 48$. 1. sing. ind. praes.

Die regelmässige endung der starken verba sowie der schwachen verba nach I und III ist $o$; bei den schw. vb. nach II herrscht grosses schwanken.

Belege für 1) starke verba: cueðo I, 51; cymo IV, 15; spreco IV, 26; uorpo VI,37; drinco XVIII,11; ongetto X,14; setto X,15; haldo VIII, 55; forleto XVI, 28; giungo VIII, 21; 
DIE SPRACHE DER NORTHUMBR. INTERLINEARVERSION. 59

Daneben begegnet $a$ bei geonga VIII, 21; aðoa XIII, 8;

$e$ bei geonge VII, 33; gesie XVI, 22; zu gisîi XX, 25; cueđi XXI, 18 s. vorwort; zweifelhaft onfoe V, 34. 41.

In anlehnung an die athematische flexion ist gebildet: geseom IV, 19; gesium XVl, 22 mit $i$ nach.2. 3. sg.; geseium IX, 25.

Die endung fehlt in forlet XIV, 18 vor folgendem ic.

2) Schwache verba I: auecco II, 19; sello IV, 14; tyrsto IV, 15; doemo V, 30;

$a$ erscheint bei doema XII, 47; aedeaua XIV, 21;

$e$ bei gemoete XVIII, 38.

Der endungsvokal fehlt vor ic in gelef ic $\mathrm{XX}, 25$, spildic XVIII, 9 .

3) Schwache verba II: igo, iga, ige begegnen in geendigo IV, 34; halgiga XVII, 19; uorđige VIII, 49;

ia in uuldria VIII, 54; ganz unregelmässig ist fulgugia I, 26; $o, a$ finden sich bei geniðro VIII, 11; lufo XIV, 31; XXI, 15; lufa XIV, 21; XXI, 16. 17;

$e$ in berhte XII, $28 \mathrm{zu}$ berhtnian.

Kontraktion fand statt bei gefeo XI, 15 .

4) Schwache verba III: neben $o$ in sago III, 3; liofo VI, 57; XIV, 19 erscheint $u$ in hafu, nafu IV, 17.

Der endungsvokal ist apokopiert vor ic: hefic IV, 17.

\section{§ 49. 2. sg. ind. praes.}

Hier herrscht mannigfaltigkeit. Bei den starken verben begegnet ausser dem gewöhnlichen -es vereinzelt auch -is und -ces, S. § 358 anm. 1. 2; schliesslich st.

Bei den schwachen verben nach I finden sich -es, -ces, auch eð; S. § 356 anm. 2; bei den schwachen verben nach II erscheint neben regelmässigem -as selten -ces; und bei den schw. verben nach III -es, -is, -as.

Belege: 1) starke verba -es: cuc®es VIII, 5 ; nimes $\mathrm{X}, 24$; cuoeðestu I, 22; uоepestu XX, $15(<\ldots s+\delta u$ S. § 201,6); -is: faris XI, 8; settis XIII, 38; -aes: uopoes XX, 13; -st: gesiist I, 33; XI, 40.

2) Schwache verba nach I: -es: cedeaues II,18; wyrces II,18; $\mathrm{X}, 33$; geheres III, 8; gelefes IX, 35; -aes: wyrcaes VI, 30; -еठ: gelefed I, 50; auecced II, 20.

3) Schwache verba nach II: -as: lufas Prf. 8,10 ; XI, 3 
fuuluuas I, 25; unas I, 38; -ces: onsucrastu XVIII, 22; lufaestu XXI, 15; S. $§ 201,6$. IV, 11 .

4) Schwache verba nach III: -es: hoefes IV, 18; -is: hoefis

$$
\S 50 .^{\bullet} 3 \text {. sing. ind. praes. }
$$

Der ursprüngliche endkonsonant $\delta$ wechselt stark und regellos mit $s$; der themavokal ist nicht synkopiert und erscheint bei starken und schwachen verben als $e$ und $a$, selten als $e$ und $i$; SS. $\S \S 357.358,2$ anm. 3.

Die belege sind sehr zahlreich.

1) Starke verba: -еð: onginneð Prf. 1, 1; spreceð Prf. 5, 6; cymeð I, 30; gebrucceð VI, 50; ongetted VII, 17; awritteð VIII, 6 ; innfared X, 9 ; onfoed XVI, 15 ; cted $\mathrm{X}, 10$; nimed $\mathrm{X}, 12$ etc.; -ed verschrieben für -eठ in astiged $\mathrm{X}, 1$; -ið bei gesiið Prf. 5, 17; 7, 5; über cuoeð IX, 41 (3. sg.?) s. L. § 107; -að: drincað II, 10 rd.; dringað Prf. 5, 7; cymað III, 8; sprecað III, 31; gebruccað VI, 54; besuiccað VII, 12; gehaldað VIII, 51 etc.; -๘ð: arisaed XI, 24; ðorføed XIII, 10; onfoæठ XVI, 14; -es: onsacces Prf. 3,4 ; gehaldes Prf. 5, 17; cymes III, 20; cuceðes VI, 42; gebrucces VI, 51; forstondes VI, 3; spreces $\mathrm{IX}, 37$; forletes $\mathrm{X}, 4$; tostraigdes X, 12; gewordes X, 16; -as: bebeadas Prf. 7, 19; stondas III, 29; forlettas X, 12; geongas XII, 19 etc.; -aes: uiðcuoеðces XIX, 12; frerces XII, 19; onfoces XIII, 20.

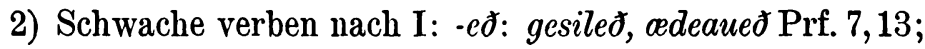
ceigeð Prf. 6, 12; gebyreð II, 4 rd.; hyncgred VI, 35; ठyrsteð VII, 37; gebirged VIII, 52; ontyneð $\mathrm{X}, 3$; sendeð $\mathrm{X}, 4$; spilleð $\mathrm{X}, 10$; spildeð XII, 25; gyrdeð XXI, 18; -ed verschrieben für -eठ bei hacled Prf. 4, 9; adeaued V, 20; -ið: wyrcið XIX, 12; selið XV, 16 ; -að: wyrcað Prf. 5,15 ; soecað IV, 27; geherað $\mathrm{IX}, 31$; loedað $\mathrm{X}, 3$; gebrengað $\mathrm{X}, 3$; gemoetað $\mathrm{X}, 9$; -es: loeres Prf. 2, 6; gewyrces Prf. 3, 3; geceiges Prf. 5, 3; Jyrstes Prf. 5, 6; getrymmes I, 15; seles VI, 27; loedes XXI, 18; -as: foestas VI, 63; wyrcas VII, 19; gehoenas VIII, 10; -ces: oferlioraes V, 24; S. § 384 anm. 3; wyrcaes XIV, 12.

3) Schwache verba nach II: -að: tobecnad Prf. 5, 17; gefoestnað Prf. 4, 3; behofað III, 7; gedœefnað III, 30; clioppað I, 15; ðerhuunað VI,27; geniðrað VIII, 10; uuldrað VIII,54; geembehtað XII,26; -iað: gewuniað XV,16; -eð: wuneठ III,36; gedaefneð III,7; -geð: deadageð VI, 50; worðageð V,23; -et: wunet VIII, 35; -as: 
DIE SPRACHE DER NORTHUMBR. INTERLINEARVERSION. 61

gebecnas Prf. 3,10; losas Prf. 4,17; tacnas Prf. 3,13 rd.; gefulguas I, 33; gesomnas IV, 36; behofas XI, 50; -ias: worđias IX, 31; uunias XII, 46; -es: uunes VI, 56; embehtes XII, 26.

4) Schwache verba nach III: -eð: gesageð Prf. 3,6; lifeð IV, 53; -ið: høefið XVI, 21; -að: gefriað Prf. 5, 15; liofað Prf. 4, 7; forhycgað V, 45; -๘ð: liofad XI, 25; -es: sages Prf. 4,11; lifes IV, 50; -is: hoefis IV, 44.

\section{$\S 51 . \quad$ Plural ind. praes.}

Hier findet ein ähnliches schwanken statt zwischen -að,

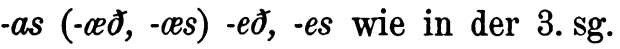

Belege: 1) starke verba: -að: arisað Prf. 4,11; сиоðаð Prf. 4, 20; geseað VI, 19; flouað VII, 38; -œð: onfoøð XVI, 24; eð: forðcymeð V, 29; genimed XI, 48; -as: onginnas Prf. 3,1 ; ongeattas Prf. 5, 14; gebiddas Prf. 7,11; gesprecas III, 11; cuceðas VII, 26; ymbcearfas VII, 22; zu cuoed IX, $40 \mathrm{vgl}$ § 50, 1; -es: uppahefes Prf. 5, 13; cymmes III, 26; astiges VII, 8; gebiddes XIV, 13; forlettes XI, 48 .

2) Schwache verba nach I: -að: adeauað Prf. 1, 10; oferleorað Prf. 4, 10; doemað V, 22; gehenað VIII, 10; fylgad X, 4;

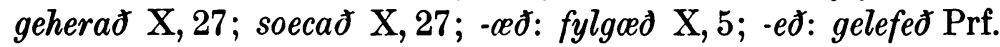
4, 5; getrymeð Prf. 4, 13; gehereð X, 16; ðer(h)trymmed X, 25; fylged $\mathrm{X}, 27$; -as: woenas $\mathrm{V}, 39$; hyhtas $\mathrm{V}, 45$; wyrcas $\mathrm{VI}, 28$; soecas VII, 19; geheras X, 3; stanas X, 32; es: getrymmes III, 11; gelefes III, 12; byges VI, 5; getrymies XV, 27; S. $§ 400$ anm. 2.

Bei invertierter wortstellung erscheint vor we eine kürzere form: wyrcae (ue) XIV, 23, das vielleicht auch als optativ zu fassen ist.

3) Schwache verba nach II: -iađ: wundriad VII, 21; genyhtsumiað VI, 7; -ias: geuorđias IV, 22; wunias XV, 7 ; -igas: bledtsigas XIII, 13; -iges: iorsiges VII, 23; -að: gewunað Prf. 5, 14; losað X,28. - Eine kontaminationsmissbildung dürfte wordaiges $\mathrm{V}, 23$ darstellen (-as, -iges).

4) Schwache verba nach III: -að: habbað VII,41; -as: habbas V, 38; giuas XV, 7; -ias: lifias XIV, 19; -es: sagces XVIII, 29. Kurzform vor ue ist nabbo (ue) XIX, 15.

\section{$\S 52$. Singular opt. praes.}

Ueber den gebrauch des optativs s. L. p. 140. Die gewöhnliche endung ist $e$, daneben begegnet $a$ und vereinzelt $a, o$. 
Beispiele: 1) starke verba: $e$ : cyme Prf. 5,6 ; sette $\mathrm{XV}, 13$; gefe XVI, 2; forlete XVIII, 39; fare XVI, 7; genimm XVII, 15; gehalde XVII, 15; a: hlada IV, 11; drinca VII, 37; gehalda XII, 7; gegripa XII, 35; aðoa XIII, 10; giunga XVI, 7; gefraigna $\mathrm{XVI}, 30$; a: genioma V, 10; auecca VI, 44; gesueltoe XVIII, 14; $o$ in cymo (?) XXI, 22; $i$ in gisîi XX, 25; S. § 374 anm. 5 .

2) Schwache verba nach I: $e$ : hreme XI, 31; doeme XII, 47; brenge XV, 2; a: gesoeca XII, 26; oferleora XIII, 1; o: spillo VI, 39; gehoelo XII, 47.

3) Schwache verba nach II: a: geuna XIV,16; deadige $\mathrm{XXI}, 23$; -ia: geendia V, 36; -ige: losige VI, 39; -iga: geunniga $\mathrm{XV}, 4$. - Kontaminationsform: losaige XI, 50, vgl. § 51, 3 .

4) Schwache verba nach III: hoebbe 188, 10.

\section{$\S 53 . \quad$ Plural opt. praes.}

Die endungen sind die gleichen wie im sing., da ursprünglich auslautendes $n$ im north. abgefallen ist.

Beispiele: 1) starke verba: $e$ : ongette $\mathrm{X}, 38$; fcre $\mathrm{XI}, 7$; toslîte XIX, 24; hleatte XIX, 24; gebrucce VI, 5; a: gedrinca VI, 53: geonga XI, 19; ae: gesea IX, 39; o: gebreco XIX, 31; $i$ in gesii $\mathrm{V}, 19$ neben gesee $\mathrm{IV}, 48$.

2) Schwache verba nach I: e: gemoete VII, 35; gebrenge $\mathrm{XV}, 8$; gelefe XX, 31: a: gebrenga XV, 16; gelefa $\mathrm{X}, 38$; gilefa $\mathrm{XX}, 31$; lacga XII, 35; ae: gelefa VI, 30; gesella I, 22; o: geuerco VI, 28; gelefo VI, 29.

3) Schwache verba nach II: -ia: wundria V, 20; losia VI, 12; -iga: lufiga XV, 12; ondspyrniga XVI, 1; gewuniga XXI, 22; -ige: gewunige XV,4; deadige XXI, 23; -age: deadage XI, 16.

Kontaminationsformen: lufaige, lufaiga XIII, 34 s. oben.

Schwache verba nach III: hoebbe $\mathrm{X}, 10$.

\section{$\S 54$. Singular des imperativs.}

Die 2. sing. imp. ist endungslos bei den starken verben, sowie bei den schwachen verben nach I, und zwar sowohl den lang- als den kurzsilbigen. Die endung bei den schwachen verben nach II ist gewöhnlich $a$, daneben findet sich -ig.

Beispiele: 1) starke verba: aris Prf. 4, 8; nim Prf. 4,8; geong Prf. 4, 9; cym I, 46; geseh I, 46; ett IV, 31; bruc IV, 31; astig IV, 49; foer VII, 3; tobar XX, 27; cuced X,24; forlet XII, 7; gehald XVII, 11; gefregn XVIII, 21. 
DIE SPRACHE DER NORTHUMBR. INTERLINEARVERSION. 63

2) Schwache verba nach I: langsilbige: hoel Prf. 6,13; soec I, 43; ceig IV, 16; gelef IV, 21; send XX, 27; foed XXI, 15; gefylig XXI, 22; vgl. § 43.

Als ausnahme begegnet: fylge I, 43 ; ced-eaua XIV, 8 ist offenbar nach analogie der schw. vb. kl. II gebildet, vgl. auch § 29 .

Kurzsilbige: sel IV, 15; byg XIII, 29; ausnahme: getrymme XVIII, 23.

3) Schwache verba nach II: a: uundra III, 7; uuldra XVII, 5; halga XVII, 17; -ig: halgig XVII, 17.

4) Schwache verba nach III: saeg XX,17.

$\S 55$. Plural des imperativs.

Das verhältnis der endungen ist das gleiche wie im plural ind.; doch überwiegen die formen mit themavokal $a$ gegenüber jenen mit $e$.

Beispiele: 1) starke verba: -að: cymað I, 39; geseað I, 39;

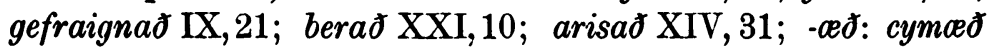
XXI, 12; -еð: cymeठ IV, 29; -as: gefraignas IX, 23; -es: lettes $\mathrm{XI}, 44$.

2) Schwache verba nach I: -að: uircað II, 5; gebrengað II, 16; bringað XXI, 10; -еð: gefylleð II, 7; dceleঠ, brengeð II, 8; -as: rehtas I, 23; uyrcas VI, 10; gedoemas VII, 24.

3) Schwache verba nach II: -ias: somnias VI, 12; -eð: birleð II, 8.

\section{§ 56. Infinitiv.}

I. Der unflektierte infinitiv hat das auslautende $n$ im north. verloren; der vokal erscheint als $a$ und $e$, vereinzelt auch a (o?).

Beispiele: 1) starke verba: a: geonga Prf. 3,15 ; begeatta Prf. 7, 11; gefara I, 43; drinca IV, 7; eatta IV, 33; cuma V, 40; ofersuida III, 14; arisa XX, 9; bidelfa XIX, 40; spreca VIII, 26; $e$ : ondrede Prf. 4, 16; slepe Prf. 6,8; gecyme V,40; geonge VII,1; gefraigne XVI, 19; gehrîne XX, 17.

2) Schwache verba nach I: a: doema Prf. 3,15 ; gewyrca Prf. 4,15; gesealla Prf. 4,18; gelefa II,4 rd.; gebrenga X,16; dryga XIII, 5; o?: raeco XIII, 26: ae: woence V, 45; gelêfoe XVII, 20; $e$ : haele Prf. 3,15; gesete Prf. 6, 3; anoecce Prf. 6,9; ge[w]yrce II, 16; geuirce III, 2; gehone VIII, 6 ; gehere IX, 27; gigyrde $\mathrm{XXI}, 18$; bybyrge XIX, 40 . 
3) Schwache verba nach II: $a$ : gemana Prf. 1,3; -ia: fulguia I, 33; geunia Prf. 4,3; uorđia IV, 24; -iga, -ega, -ige, -ege, -age, -ge: lufiga VIII, 42; uuniga XXI, 23; synngega VIII,11; lytlige Prf. 4,2; gebrehtnige Prf. 6,17; synngige V,14; deadege IV, 47; gelosage X,39; geworðage IV,20; gefeage III,14 (s. S. $\S 391$ anm. 6); fulguge Prf. 4, 1.

Kontaminationsbildung ist uundraige $\mathrm{V}, 28$.

$\mathrm{Zu}$ geuuni XXI, 22 s. vorwort.

4) Schwache verba nach III: gefiage VII, 7.

II. Als endung des flektierten infinitivs erscheint -anne (auch -ane geschrieben), daneben vereinzelt -enne.

Beispiele: 1) starke verba: -anne: redanne Prf. 4, 19; gesaccanne Prf. 8, 4; zu awrittanni XXI, 25 s. vorwort; -ane: underniomane Prf. 8,2; sprecane VIII, 26.

2) Schwache verba nach I: -anne: gelefanne Prf. 4,2; geladanne Prf. 6, 4; halanne Prf. 6, 19; wircanne II, 4; -ane: gestcenane XI, 8; -enne: doemenne Prf. 6, 18.

3) Schw. verba nach II: lufanne Prf. 7, 16; fulguanne I, 31.

4) Schwache verba nach III: saggganne XVI, 12; forhygcanne Prf. 7, 12.

$\S$ 57. Participium praes.

Die endung ist normaler weise -ende, daneben begegnet vereinzelt -ande und einige mal -end.

Beispiele: 1) starke verba: -ende: niomende Prf. 3,6; cuoeðende I, 15; scinende V, 35; woepende XI, 33; stondende XI,56; -ande: beadande Prf. 8,11; -end: spreccend VIII, 12; sprecend XII, 29.

2) Schwache verba nach I: -ende: laedende Prf. 3,6; lixende V, 35; hremende XI, 33; untrymiende Prf. 4, 6; s. § 400 anm. 2.3 -ande: adeauande XIV, 22; -end: sellend VI, 71.

Ueber fylgendi XXI, 20 s. vorwort.

3) Schwache verba nach II: (ig) ende (-a): tceherende XI, 35; frasendum Prf. 3,4 ; cliopendes Prf. 3,5 ; wuniende XIV, 25; wunigendoe I, 33; -ande: fuluande Prf. 3, 7; 4,1; fulguande I, 28; clioppande VII, 28.

Ueber hlingindi XXI, 12 s. vorwort.

4) Schwache verba nach III: -ende: lifigiende VII, 38; -end: habend Prf. 4, 8; habbend $\mathrm{X}, 21$; -and in den substantivierten: londbucend Prf. 7,9. 
DIE SPRACHE DER NOR'THUMBR. IN'TERLINEARVERSION.

§ 58. Sing. ind. des starken praet. $e$ aus.

Die 1. und 3. sing. sind endungslos, die zweite geht auf

Beispiele 1. und 3. sing.: bebead Prf. 1, 4; ongann Prf. 1, 6; awrat Prf. 1,11; gebrcec XIII, 26; baedd XIX, 38; onsôc I, 20; onfeing XIII, 4.

2. sg.: e: gehealde II, 10; gecuome III, 2; cuede IV, 17.

Irrtümlich steht $e$ in gestode (3. sg.) I, 35, wahrscheinlich durch den dental der wurzel zur schwachen bildung veranlasst.

$\S 59$. Plural ind. des starken praet.

Es wechseln die endungen: -on, -un, vereinzelt -en. Das auslautende $n$ bleibt in der regel, doch fällt es vor unmittelbar folgendem pronomen (-ue, -gie) ab; ausserdem begegnet noch zweimal vokalische endung.

Beispiele: -on: onfeingon I, 11; ueron Prf. 1,14; cuomon Prf. 8, 5; gesegon V, 37; cucedon VIII, 57; fornomon I, 5; gefrugnon V, 12; getugon VI, 52 etc. -un: genomun IV, 45; gwurnun IV, 51 ; ongetun VII, 26; frugnun VIII, 7; gesegun III, 11; getugun IX, 22; stodun XI, 56; cuoedun XII, 29 . -en: cuoeden VIII, 4 ; uuunden XIX, 2 . o vor -gie: ongetô VIII, 55; ongetto XIV, 9; ohne folgendes pronomen: gesego IX, 41; cuomo XIX, 32.

$\S 60$. Sing. ind. des schwachen praet.

Die endung der 1.3. sg. ist $e$, daneben vereinzelt $-\infty e, o$; die 2. sg. geht auf -es, selten -est aus.

Beispiele: 1) schwache verba nach I: 1 . 3. sg. e: eftgeceigde Prf. 1, 3; foerde VIII, 1; at-eaude, gecydde Prf. 1,5; gesette Prf. 1,7; sende XVII, 18 etc. -a: leerdae VII, 28; reteuwdae XXI, 14 ; cudoe 188, 3 . o: getrymmedo XII, 17.

$\mathrm{Zu}$ girasti XXI, 20; gihamadi 188, 7 vgl. vorwort.

Der endungsvokal ist abgefallen vor folgendem $-i c$ bei: spildic XVIII, 9; er fehlt ferner bei: yeonduard X, 32; worlht $\mathrm{XXI}, 25$.

2. sg. -es: geherdes XI, 41 ; sendes XI, 42 ; foerdes XVI, 30 . -is: getrymedis III, 26.

2) Schwache verba nach $\Pi:$ 1. 3. sg. gelytlade Prf. 1,9; geuitgade Prf. 2, 1; uorðade VIII, 49.

Der endvokal fehlt bei: cliopad XI, 43; lufad XV, 12 .

Anglia. N. F. $\mathbf{X} I$. 
2. sg. -es: lufades XVII, 23.

3) Schwache verba nach III: 1. 3. sg. e: sagde I, 15; haefde Prf. 4, 2; lifde IV, 51 . o: saegdo III, 28.

2. sg. -es: gegiuuedes IV, 10; nafdes XIX,11. -est: hafdest IV, 18; scegdest IV, 18.

$\S 61$. Plural ind. des schwachen praet.

Die normalendung ist -on, daneben begegnet nicht selten -un; abfall des $n$ findet sich zweimal bei zwei nebeneinander stehenden formen, von denen die eine unmittelbar vor -ue steht, und die andere offenbar nach ihr gebildet ist, ausserdem bei uoendo XIII, 29.

Beispiele: 1) schwache verba nach I: -on: gelefdon Prf. 4, 10; gesendon I, 19; gemoetton I, 41; geherdon V, 37; woendon XI, 13 . -un: gebrohtun II, 8; gemoetun I, 45; geondueardun VIII, 33; uorhtun XII, 2.

2) Schwache verba nach II: -on: tuiaton XIII, 22; gewordadon IV,21; gegeadredon Prf. 6,10. -un: gelufadun III,19; gewordadun IV,20; sticadun XIX,37. e: bloedsade(ue); sagnade VIII, 48; s. oben. $\mathrm{XV}, 24$.

3) Schwache verba nach III: -on: sagdon IV, 51; gefiadon

§ 62. Singular opt. des praeteritums.

Die regelmässige endung ist bei starken und schwachen verben $e$.

Beispiele: 1) starke verba: cuome XV,22; ofercuome Prf.1,14; onsoce Prf. 8,11; onfenge XIII, 30; gehealde Prf. 1, 5 .

2) Schwache verba nach I und III: gesalde Prf. 3,13; sagde XIV, 2.

§ 63. Plural opt. des praeteritums.

Gegenüber der ws. endung auf -en erscheint $e$ und häufiger noch $o$, letzteres in anlehnung an den indikativ. S. $\S 365$ anm. 2.

Die vollformen auf -en und -on sind selten und in ihrer geltung als optativ auch zweifelhaft; s. L. § 121.

1) Starke verba -en: gesegen IV, 45 (opt.?). o: gesego XII, 9 ; genomo VI, 15; gegrioppo. VII, 32.

2) Schwache verba nach I und III: -on: naefdon XV, 24 . $o$ : geworhto VI, 15; noefdo IX, 41 . e: gelefde V, 46. 
DIE SPRACHE DER NORTHUMBR. INTERLINEARVERSION.

$\S 64$. Participium praeteriti.

I. Das part. praet. der starken verba endet auf -en; daneben begegnet -an, an und $n$ mit synkope des themavokals.

Beispiele: gecoren Prf. 1, 2; geworden Prf. 1,6; auritten II, 17; gehaten Prf. 3, 11 etc. -an: geðucen XIII, 10. -an: ahaldan V, 4 ; asceadan Prf. 8,1. $n$ : gesegn I, 50.

II. Das part. praet. der schwachen verba endet auf $d$ mit entsprechendem mittelvokal; (s. unter stammbildung der schw. vb.) auf $t$ bei denen ohne mittelvokal nach velarem konsonanten.

Beispiele: gecyðed Prf. 1, 7; genemned Prf. 3, 9; gehoeled Prf. 4,6; aedaued Prf. 4,4; I, 31; gelaðad Prf. 1,9; gedoefnad Prf. 2, 4; gesigfastnad Prf. 8,12; geuuldrad VII, 39; gescegd Prf. 3, 3; asogd XXI, 25; geworht Prf. 3,2; gesoht Prf. 4, 17; unbocht XV, 25.

$\delta$ für $d$ findet sich bei ofergesetteð $\mathrm{XI}, 38$.

$t$ ist geschrieben bei geinsetet Prf. 1, 10; gesettet Prf. 2, 2; ingemoetet Prf. 2, 3; gesendet Prf. 3,5; gedeðet XII, 33.

Kapitel XIV. Tempusbildung der starken verba.

I. Ablautende verba.

$\S 65$. Klasse I.

1) Der vokal des praesensstammes ist $\bar{\imath}$.

Belege: biddendra V, 3; todrifed X, 12; gegripa XII, 35; lerining Prf. 7, 3; gehrine XX, 17; arisad Prf. 4, 11 (ind. pl.); arisad XIV, 31 (imp. pl.); arised II, 22; VII, 52; arisa XX, 9; gerised III, 7 ; aris Prf. 4, 8; gescined I, 5 ; scinende V, 35; toslîte XIX, 24; besuicad VII, 12; astigo XX, 17; astiged X, 1; astiges VII, 8; astig IV, 49; stigende I, 32.33; auritteð VIII, 6; auritte XIX, 21.

Schwundstufig und infolge dessen dem o/a-umlaut unterworfen ist der vokal bei: gegrioppanne Prf. 5, 5; hrioppað IV, 36 [neben gegripa, s. oben]. S. § 382 anm. 3.

2) Der vokal der 1.3. sg. praet. ist $\bar{a}$.

Belege: abad VIII, 9; fordraf II, 15; gehran Prf. 8, 7; arâs II, 22; XI, 29; astāg VI, 17; gestag Prf. 2,1; awrat Prf. 1,11; aurât VIII, 8. 
Eine neubildung nach der schwachen flexion ist ofdune stag ade $\mathrm{V}, 4$. S. § 382 anm. 4.

3) Der vokal der 2. sg., sowie des pl. ind. und des ganzen opt. praet. ist $i$; dem pl. ind. kommt $u$-umlaut zu.

Belege: pl. ind. mit $u$-umlaut: fordriofon IX, 34; flioton $\mathrm{IX}, 22$; -io ist durch palatalumlaut vereinfacht bei: ofstigon VI, 16 ; s. $\$ 19,5$; opt. stige IV, 47 ; gestige V, 7 ; u-umlaut ist eingedrungen in: gegrioppo VII, 32 (opt. pl.).

4) Der vokal des part. praet. ist $\breve{\imath}$.

Belege: fordrifeno XI, 42 ; begrippene Prf. 5,8 ; tosliten XXI, 11; unslitten XIX, 23; besmitten XVIII, 28; besuicen VII, 47; uriten VI, 31; auritten II, 17.

\section{§ 66. Klasse II.}

1) Der stammvokal des praesens ist - $\bar{e} a$, daneben $-\bar{e} o$, s. $\S 13$.

Belege: -ea: bebeado XV, 14; bebeadas Prf. 7, 19; beadande Prf. 8,11; hlcatte XIX, 24; -ea erscheint auch in den verba contracta: fleas X,5; getea XXI,6, § 22,2. -eo: bebeodo XV,17. $i$-umlaut liegt vor bei dem verbum contractum fliid $\mathrm{X}, 12$ (<*fliohid - *flihid). S. § 371 anm. 5. $\bar{u}$ als praesensvokal erscheint in bruc IV, 31; bruccað VI, 56; bruced VI, 57; gebrucces VI, 51; gebrucced VI, 50; gebruccað VI, 54; gebrucce VI, 5. 53; s. Kluge, P. G. § 161.

2) Der vokal der 1.3. sg. praet. ist -ea.

Beispiele: bebead Prf. 1, 4; geceas VI, 70; gecêas XIII, 18. Infolge palatalumlauts ist vereinfachung $\mathrm{zu} \bar{e}(\boldsymbol{x})$ eingetreten bei fromgebeg V, 13; gebeg VIII, 6 ; gebêg XX, 5 ; geflah VI, 15; s. § 19, II.

Offenbar verschrieben ist ofgoet II, 15 für -geat; s. L. § 125, II, doch vgl. auch $\S 19$, II.

3) Der stammvokal der 2. sg. ind., des plurals ind., sowie des ganzen opt. praet. ist $u$.

Beispiele: getugon VI, 52; getugun IX, 22; XXI, 8.

Der vokal des singular ist eingedrungen bei gebrecon VI, 26. 49; XVIII, 28; gebrêcon VI, 58; gebrêicon VI, 31; s. §§ 19,II; 13 ; geceason XV, 16.

4) Der stammvokal des part. praet. ist $o$.

Belege: gecoren Prf. 1, 2, mit grammatischem wechsel; gotteno Prf. 6, 11. 
$\S 67$. Klasse III.

1) a) Bei den verben auf nasal + konsonanz ist der stammvokal des praes. $i$; s. $§ 3$.

Belege: drinco XVIII, 11; drinca VII, 37; drincað II, 10 rd.; đlringad Prf. 5, 7; gedrinca VI, 53; blimpe V, 14; onginneð Prf. 1, 1; gesingad XIII, 38.

Hierher gehören auch mit $r$-metathese und nachfolgender brechung: iornendum Prf. 8,3; bearnende V, 35; s. § 16, 1 .

b) Bei den verben auf $r+$ konsonanz wird der ursprüngliche praesensvokal zu (eo), ea gebrochen; unter einfluss eines vorausgehenden $w$ erscheint $o ; \mathrm{s}$. $§ 20$.

Beispiele: cearfanne Prf. 5,3; gecearfa VIII, 37; ymbcearfas VII, 22; uorpo VI, 37; geuordes X, 16; geuorða III, 9.

c) Bei den verben auf $l+$ konsonanz erscheint der praesensvokal in seiner ursprünglichen gestalt $e$ : bidelfa XIX, 40; helpend subst. V, 7; gesuelta IV, 47; gesueltce XVIII, 14.

d) $e$ erscheint als praesensvokal vor $-g n,-g d$ bei den verben: gefregn imp. XVIII, 21; gefregne XVI, 5 ; daneben gefraigna $\mathrm{XVI}, 30$; gefraigne XVI, 19; gefraignad IX, 21; gefraignes XVIII, 21; gifroegna XXI, 12; tostraigdes X, 12 zur schreibung, vgl. § 14 .

e) Schwundstufiger wurzelvokal $u$ begegnet im praes. bei ondspyrneđ VI, 61, Kluge P.G. $§ 161$; wegen des umlauts s. S. $\S 416$ anm. $11 \mathrm{e}$.

2) a) Der stammvokal der 1. 3. sg. ind. praet. der verba auf nasal + konsonanz ist $a$.

Beispiele: dranc IV,12; ongann Prf. 1,6; gesang XVIII,27; gesıanng XIX, 1 ; mit $r$-metathese gehören hierher: forearn $\mathrm{XX}, 4$; gearn Prf. 6,12; giarn XX, 2. S. § 386 anm. 3 .

Der vokal des plurals ist eingedrungen bei gisprunt 187,14.

b) Bei den verben auf $r+$ konsonanz wird der stammvokal der 1 . 3. sg. ind. praet. $a$ zu $e a$ gebrochen; nach vorausgehendem $w$ findet sich $a(a)$, s. $\S 20$.

Beispiele: ofcearf XVIII,10; warp Prf. 3,12; ward Prf. 2,2; geward Prf. 6, 5; geucerठ I, 17.

c) Bei den verben auf -gn, -gd erscheint als stammvokal der 1. 3. sg. ind. praet. $a$ : gefragn IV, 52; gefraign XVIII, 7; gebrogd XVIII, $10 ;$ s. $§ 1,1 ; \S 14$. 
Bemerkung. Der vokal des praes. (unter $w$-einfluss) erscheint im opt. suoelte XI, 37; das in anlehnung an die schwache flexion gebildet ist. S. $§ 387$ anm. 6 .

3) Der stammvokal der 2. sg. ind., des pl. ind., sowie des ganzen opt. praet. ist $u$.

Beispiele: a) verba auf nasal + konsonanz: wunnon IV,38; gebundun XVIII, 12; gewundun XIX, 2; uuunden XIX,2; verba mit $r$-metathese: geurnun $\mathrm{XX}, 4$; gwurnun IV, 51 .

b) verba auf $r+$ konsonanz: auurpon VIII, 59; IX, 34; $o$ ist eingedrungen in auorpon IX, 35 .

c) verba auf -gn: gefrugnon I, 19; V,12; frugnun VIII, 7.

4) Der stammvokal des part. praet. ist $u$ vor $n+$ konsonanz; sonst 0 .

Beispiele: a) Vor $n+$ konsonanz: gebundeno XI, 44; indrungno II, 10; gesungen Prf. 2, 2.

b) Vor $r+$ konsonanz: toworpen XI, 52; gramm. wechsel haben: geworden Prf. 1,6; auorden V, 14.

c) Vor $l+$ konsonanz: gedolfene Prf. 2,1 .

d) Vor -gd: tostrogden XI, 52.

\section{$\S 68$. Klasse IV.}

1) Der stammvokal des praes. ist $e$, das ev. o/a-umlaut zu ea erleidet.

Beispiele: berende XIX, 5 ; unberende XV, 2 rd.; berað XXI, $10 ;$ gestele $\mathrm{X}, 10 ;$ o/a-umlaut bei gebeara XVI, 12 . ae wohl aus dem sing. praet. eingedrungen findet sich bei tobar $\mathrm{XX}, 27$; gebraccgad XIX, 36.

Ueber die formen von ws. cuman und niman s. unten.

2) Der stammvokal der 1.3. sg. ind. praet. ist $a$ : gebracc XIII, 26; geboer XIX, 5 .

3) Der stammvokal der 2. sg. ind., des pl. ind., sowie des ganzen opt. praet. ist $\bar{e} ; \mathrm{s.} \S 6$ : gebrêcon XIX, 32; gebreco XIX, 31 .

4) Der stammvokal des part. praet. ist $o$ : eftboren III,5; tobroccen XXI, 11; tobrocene XIII, 26; tobroceno XIX, 31.

Besondere behandlung erheischen die verba ws. cuman und niman. Bei cuman erscheint weitaus häufiger als $u y$ als praesensvokal, das nach beseitigung des umlauts der 2. 3. sg. S. § 371 anm. 1 und 5 eigentlich nur dem athematischen optativ cyme, Kluge, P.G. $\S 166$, zukommt. Es begegnen folgende 
formen: cuma V, 40; VII, 36; cummende 1, 29; cume VII, 34; opt. cyme Prf. 5, 6; VII, 37; cymo XXI, 22; ind. cymo IV, 15; V, 7; cymeð I, 30; IV, 29; cymmed III, 21; cymað I, 39; III, 8; cymes III, 20; cymmes III, 26; cymas XIV, 23; imp. cym I, 46; XI, 34; cymm XI, 43; cymmende I, 9; tocymende I, 15; forðcymed $\mathrm{V}, 29 ;$ gecyme $\mathrm{V}, 40$.

Der stammvokal des ganzen praeteritums ind. und opt. ist $\bar{o}$ : cuom Prf. 5, 5; 7,17; I, 30; 2. sg. gecuome III, 2; cuome VI, 25; opt. cuome XV, 22; ofercuome Prf. 1, 14; cuomon Prf. 8, 5; I, 39; cuômon XXI, 8; cuommon XVIII, 20; cuomo XIX, 32; S. §390 anm. 2.

Der stammvokal des part. praet. ist $u$ : cummen XVI, $32 \mathrm{rd}$. s. $\S 21$, I.

Der praesensvokal von niman ist $i<\ddot{e}$ vor einfachem nasal, $\S 15,5$. Die formen mit und ohne o/a-umlaut sind nicht mehr streng geschieden, sondern es haben nach beiden richtungen hin ausgleiche stattgefunden. Unverändertes $i$ begegnet bei: nim Prf. 4, 8; XIX, 15; nimed I, 29; VIII, 37; X, 12; nimes X,24; genimma VI, 44; genimme XVII, 15; XX, 15; ferner bei nimað $\mathrm{XX}, 23$; nimo XII, 32. io $<i$ durch o/a-umlaut bei: niomad $\mathrm{XI}, 39$; genioma X, 29; underniomane Prf. 8,2; ferner bei: geniome V, 10; niomende Prf. 3,6; II, 6 .

Der stammvokal des ganzen praeteritums ind. und opt. ist $\overline{0}$ : S. § 390 anm. 2: genom V, 9 ; fornomon I, 5; genome XIX, 38; $\mathrm{XX}, 15$; genomo VI, 15.

Der stammvokal des part. praet. ist $u<0$ vor einfachem nasal, § 15, 5. (be-), for-, numene Prf. 1, 7; ofnumen VIII, 3 .

\section{$\S 69$. Klasse V.}

1) Der stammvokal des praes. ist ursprünglich $e$, das aber nach den regeln von $\S 18$. a. b. $\S 20$. I. V häufig zu $(e o), e a, o e$, $o$ verändert wird. Bei den verben mit praesentischer $j$-ableitung erscheint $i, \mathrm{~S} . \S 391,3$.

Belege sind: сиеðо I, 51; сиеði XXI, 18; gесиеðаð II, 5; cuoeðеstu I, 22; uið-cuoeðқes XIX, 12; cuoeðende Prf. 5, 7; I, 15; cuoeðendes Prf. 4,7; cuoedo XIV,2; XVI, 26; cuoedendo Prf. 4,5 mit $d$ aus den formen mit gr. wechsel. Ueber cuoed IX, $41\left(^{2}\right)$ s. $§ 50,1 ; \S 51,1$; сиоðаð Prf. 4, 20.

$a$, wohl aus dem praeteritum eingedrungen, erscheint bei

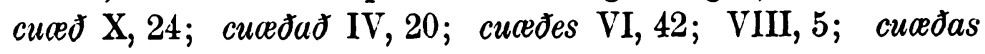


VII, 26; IX, 19; cuceðende Prf. 7, 9; - -eteð X, 10; eatta IV, 33; eatas XXI, 12; eattanne VI, 52; gefe XVI, 2; forgefes XX, 23 ; ongette VII, $51 ; \mathrm{X}, 38$; ongetted VII, 17 ; ongetto $\mathrm{X}, 14$; ongeattas Prf. 5,$14 ;$ VIII, $43 ; \mathrm{X}, 14 ;$ begeatta Prf. 7, 11; spreco IV, 26; sprecco XII, 50; spreced Prf. 5, 6; spreces, sprecces XVI, 13; sprecað III,31; spreccende I,37; sprcccend VIII, 12; gesprecas III, 11; tuispreccendo Prf. 4, 20.

$a$ erscheint in spracende VI, 63; spraccend XVIII, 21.

Die verba mit praesentischer $j$-ableitung haben $i$ als wurzelvokal.

Beispiele: bidda XVI,26; gebiddo XIV,16; gebiddes XIV,13; gebiddas Prf. 7, 11; licgende V, 6; sittendo II, 14; gesitta VI, 10.

Umlauts $-i$ in der 2.3. sg. hat meist das verbum contractum sēa, S. § 371 anm. 5: gesiið Prf. 5, 17; gesiis III, 32; gesiist I, 33; $\mathrm{XI}, 40$; einmal geseað IX, 21. Sonstige formen dieses verbums sind: 1. sg. gesium, gesie XVI, 22; geseium IX, 25; geseom IV, 19; pl. geseað I, 51; imp. geseh I, 46; XI, 34; XX, 27; gesagh VII, 52, § 19, III; geseað I, 39; opt. gesee IV, 48; gesii V, 19; gisîi XX, 25; inf. gesea Prf. 6,14; XII, 21.

2) Der stammvokal der 1.3. sg. ind. praet. ist $a$, das nach den regeln von $\S 20, \mathrm{IV} ; \S 21, \mathrm{II} ; \S 19$, I häufig zu oe (oce), ea $(e a), a$ (über $e a$ ) verändert wird.

Beispiele: сисљ Prf. 3,$16 ; \mathrm{XX}, 15 ;$ gесисе XI, 43; сиосð Prf. 3, 14; 4,16; coeð XVIII, 37; gecuoed Prf. 4, 8 mit $d$ nach dem plural; forecuoced Prf. 7, 12.

$e$ nach dem praes. erscheint in cued Prf. 4,18; XX, 15; s. auch S. $\S 391$ anm. 10; bcedd XIX, 38; becedd XIX, 12; gebced $\mathrm{V}, 47$; onyoett VI, 15 ; ongoet XII, 9 ; ongeat $\mathrm{I}, 10 ; \mathrm{IV}, 1 ;$ gelceg V, 3; gesah I, 18. 29. 32 mit palatalumlaut; gesat Prf. 6, 13; gesaett IV, 6; sprac Prf. 4, 4; gesprac Prf. 7, 18; sprace $\mathrm{X}, 6$; $\mathrm{XIV}, 25$.

Ein reduplikationsprodukt ist der vokal in geêt II, 17; S. $\S 391$ anm. 3; Kluge, P.G. § 171, p. 436.

3) Der stammvokal der 2. sg. ind., des pl. ind., sowie des ganzen opt. praet. ist $\bar{e}, \S 6$, das nach $\S 20$, III zu oe verändert werden kann.

Beispiele: mit gr. wechsel cuede IV, 17; cucdon II, 18; gecuede VII, 9; cuoedon I, 22; cuôedon XIX, 6 ; cuoeðon XVIII, 7 mit falschem $\delta$ nach dem sing. 
ce nach dem sing. erscheint in cucedon VIII, 57;

bedon IV, 31; gebêdon XIX, 31; geeton VI, 13; geêton VI,23; ongeton VIII, 52 ; ongetun VII, 26 ; ongetton VI, 69 ; ongêto VIII, 55 ; gesege (2. sg.) VIII, 57 ; gesege (opt.) VI, 5; VIII, 56; gesegen IV, 45; gesego IX, 41; XII, 9; gesegon Prf. 8, 8; I, 14. 39 etc.; gesêgon XXI, 9; gesegun III. 11, bei diesem verbum gr. wechsel; seton $\mathrm{XX}, 12$; aseton VIII, 3 ; missprecon VI, 41.

ae in gesprace IV, 27.

4) Der stammvokal des part. praet. ist $e$, das nach $\S 20$, I zu oe verändert werden kann.

Belege: acueden XIX, 30 rd.; gecuedne Prf. 1, 13; forcuoeden Prf. 6,12; forcuoedne Prf. 7, 13; gecuoeden Prf. 4, 17; forgefen XX, 12; ongeten Prf. 8, 4; forrepene Prf. 5, 8; S. § 391 anm. 1; gesegn I, 50; geuoefen XIX, 23.

\section{$\S 70$. Klasse VI.}

1) Der stammvokal des praes. ist $a$, das vor folgendem palatalen vokal zu $a$ erhöht wird und analogisch auch vielfach vor velarem vokal als $a$ erscheint. Vor folgendem nasal wird $a \mathrm{zu} o$ verdumpft.

Beispiele: fara VI, 67 ; gefara $\mathrm{I}, 43$; innfared $\mathrm{X}, 9$; fore XVI, 7; faere XI, 7; far VII, 3; farende VII, 35; S. § 50 anm. 1; faeris XI, 8; faro XIV, 3; XVI, 7; farað III, 8; lâdanne IV, 7; gehladanne IV, 15; S. §50 anm. 1; onsaeces Prf. 3,4; XIII, 38; saccendum Prf. 6,14; gcsaccanne Prf. 8,4; S. §50 anm. 1; stondas III, 29; stondende XI, 56; XVIII, 18; stôndende XVIII, 16; forstondes VI, 63 ; ymbstondas XI, 42; auexe III, 30 mit palatalumlaut.

Das verbum contractum sléa weist in der 2. 3. sing. ind. $i$-umlaut auf; S. $§ 371$ anm. 5: slces XVIII, 23; of slaed VIII, 22; XVI, 2; inf. geslea XVIII, 31 .

Praesentische $j$-ableitung und infolgedessen $i$-umlaut im ganzen praesens-stamm hat das verbum hebban (< hafjan) s. $\S 37$; ahefes Prf. 5, 13; ahefað IV, 35; ahefed VIII, 28; XIII, 18; wo $f$ jedenfalls aus dem praet. bezogen ist; hebbendum XI, 41; underhebendum XVII, 1 ; s. $\S 37$.

Ueber die praesensformen von (a)ðoa vgl. $\S 22,1$; ađuah IX, 7 ist ein analogisch gebildeter imperativ für *aðuceh; $\mathrm{S}$. $\S 162,1$. 
2) Der stammvokal des ganzen praeteritums ind. und opt. ist $\bar{o}$. Belege: drog XXI, 11; drogun XXI, 8; underhof V, 9; slog XVIII, 10; geðuog XI, 2; aðuog IX, 7; geðuoh Prf. 7, 1; S. $\S 392,2$; onsôc I, 20; XVIII, 25; onsoce Prf. 8, 11; stód VIII, 44; gestod I, 26; gestode I, 35; ofstod VI, 18; stodun XI, 56; gewox Prf. 4,1 ; S. $§ 392$ anm. 5 .

3) Als stammvokal des part. praet. erscheint $c e$ und $e$, vor nasalen 0 .

Beispiele: ahoefen XII, 34; ahefen XII, 32; geむucen XIII, 10; S. § 368 anm. 4; uiðstonden Prf. 5, 7.

II. Reduplizierende verba.

Von den bei S. $\S \S 394-396$ angeführten reduplizierenden verben erscheinen in unserm texte folgende formen.

\section{$\S 71$. Praesens.}

ondreded (3.sg.) XIV, 27; ondrede (inf.) Prf. 4, 16; VI, 20; redanne Prf. 4, 19; redendum Prf. 1, 8; s. S. § 395 anm. 3; forleto XVI, 28; forletto XIV, 27; forlet XIV, 18 (1. sg.); lettes XI, 44; XVI, 2; forleted VIII, 29; forlettas X, 12; forletas XVI, 32; forletes X, 4; forlet (imp.) XII, 7; forleta Prf. 7,8; XIX,10; forletanne XIX, 10; slepeð XI, 11; haldo VIII,55; gehaldað VIII,51; gehalded VIII, 52; gehaldas XV, 20; gehaldes Prf. 5, 17; gehalde XVII, 15; haldendum Prf. 7, 7; gehald (imp.) XVII, 11; gehala XIX, 16, wohl verschrieben für gehald; geonge VII, 33; geonga VIII, 21; XI, 11; giungo VIII, 21; geongas XVI, 5; gegeongað $\mathrm{XI}, 10$; (ind.) geonga XV, 16; giunga XVI, 7 (opt.); geong (imp.) Prf. 4, 9; VII, 3; geongende Prf. 4, 16; I, 23; geonga (inf.) Prf. 3.15 ; uоepoes $\mathrm{XX}, 13$; uoepestu $\mathrm{XX}, 15$; uoepende XI, 33; saueठ IV, 36; flouad VII, 38.

\section{§ 72. Praeteritum.}

Alte reduplizierte praeterita sind bewahrt in: ondreard XIX, 8; ondreardon VI, 19; IX, 22; forleort IV, 3. 52; geheht Prf. 6, 9; 7, 7; redon XIX, 20 (korrig. aus reddon, s. S. § 395 anm. 3); feoll VI, 31 rd.; IX, 38; feall XI, 32; fcollon XII, 13; XVIII, 6 ; gefealla XII, 24 ; s. $\S 13$; beheald I, 36; geheald XVII, 12; gehéald XV, 10; gehealdon XV, 20; gehealde Prf. 1, 5; II, 10; hroeuun VI, 19; S. § 396 anm. 3; gewoep XX,11; gebleou XX,22; oncneaun XII, 16. 
Eine interessante form ist blefla $\mathrm{XX}, 22$ : professor Schick erblickt darin in übereinstimmung mit professor Paul ein altes redupliziertes praeteritum $\mathrm{zu}$

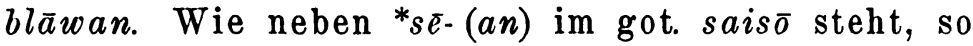
könnte zu einer wurzel *ble-das praeteritum *be-blo gebildet sein, das sich im ae. regelrecht zu ${ }^{*} b e f l a$ weiter entwickelt (vgl.hona ${ }^{*}$ hanõ). Die auffällige wiederholung des $l$ in der reduplikationssilbe lässt sich vielleicht durch analogische beeinflussung vom praes. blawan erklären. Somit liegt wohl kein bedenken mehr vor, blefla als einziges praeteritum mit vollkommen erhaltener reduplikation an die spitze der fünf übrigen formen des ae. zu stellen, die die alte reduplikationsbildung noch deutlich erkennen lassen.

$\S$ 73. Participium praeteriti.

forletno Prf. 7,17; gchatne Prf. 1, 14; gehaten Prf. 3, 11; 7,8 ; asceadan Prf. 8, 1; gefâlden XX, 7; ahaldan V, 4 ; gehalden Prf. 2, 6; gehaldeno XX, 23; forblauene VI, 18.

Von den verba contracta fo $a$ und hoa sind folgende formen belegt:

1) Praes. ind. sg. onfoe ic V,34; onfoed XVI, 15; onfoced XVI, 14; onfoas III, 32; onfoes III, 33; pl. onfoced XVI, 24; onfoas Prf. 3, 3; III, 11; V, 43; onfoes V, 43; opt. onfoe VI, 7; inf. onfoa III, 27; VI, 21; onfoanne Prf. 6, 5; dieser infinitiv ist eine analogische neubildung nach den übrigen infinitiven auf - $a$ (helpan, onfōn > helpa, *onfo > helpa, onfoa); onfanne X, 18; pt. foendo Prf. 4, 13.

2) Praet. ind. sg. onfeng VI, 11; XIX, 27; onfeing XIII, 4, s. $\S 14$; pl. onfengon I, 12. 16; V, 44; XVII, 8; onfeingon I, 11; s. § 14; opt. onfenge XIII, 30; XVIII, 3; gefengo XI, 57.

3) Part. praet. befoen VIII, 3.

1) Praes. inf. gehoa XIX, 10; hoanne XIX,10; ahoanne XIX, 16; s. oben zu foa; opt. ahoa XIX,15; imp. ahoh XIX,6( $\left.{ }^{2}\right)$. 15; ahoad XIX, 6.

2) Praet. ind. pl. ahengon XIX, 18. 23.

3) Part. praet. ahoen XIX, 16. 20. 32. 41. 
Kapitel XV. Tempusbildung der schwachen verba.

\section{$\S 74$. Klasse I.}

1) Praesens. Ursprünglich kurzsilbige. Alle formen des praesensstammes haben $i$-umlaut und verdoppeln mit ausnahme der 2. 3. ind. und 2. sg. imp. den wurzelauslautenden konsonanten - abgesehen von denen auf $-r$ unter verlust des ableitenden konsonanten.

Beispiele: bebycgendo II, 14; sello IV, 14; setto XIII, 37; gesellae I, 22; auecco II, 19; pl. derhtrymmes III, 28 etc.; 2.3. sg. aðenes XXI, 18; seleむ X, 11; gesileむ Prf. 1, 4; silið XVII, 2; XXI, 13; gesilið XI, 22; wegen $i$ s. § 21, VI; getrymeð Prf. 4, 3. Doch sind die verhältnisse nach beiden richtungen hin mannigfach verschoben.

Ohne lautgesetzliche gemination begegnen: selo IV, 14; VI, 51; XIII, 34; silo X, 28; eftaueco VI, 39; pl. byges VI, 5; getrymed V, 36; getrymes III, 11; inf. gesete Prf. 6, 3 .

Nach den verben auf $-r$ sind gebildet: untrymiende Prf. 4,6 ; pl. getrymies XV, 27 ; S. $§ 400$ anm. 2. 3 .

Andrerseits erscheint häufig gemination auch in der 2.3. sg. und in 2. sg. imp.: settis XIII, 38; setted Prf. 3, 14; II, 10; geselled XIII, 21; auecced II, 20; auccced V, 21; imp. getrymme XVIII, 23.

Zum inf. gesealla Prf. 4, 18 etc. mit o/ $a$-umlaut vgl. § $18 \mathrm{~b}$.

Die verba auf $-r$ bewahren in allen praesensformen den einfachen konsonanten am ende der wurzelsilbe und verlieren das ableitende $j$ nur in der 2.3. sg. und 2. sg. imp.

Beispiele: byreð X, 13; gebyreð II, 4 rd.

Ursprünglich langsilbige. Der ganze praesensstamm hat $i$-umlaut; das ableitende $j$ fällt aus ausser im verbum ceiga, wo es in allen formen erhalten ist.

Beispiele: gewyrces Prf. 3,3; lceres Prf. 2,6; gelefeð Prf. 4,5 ; soecendum Prf. 2, 5 ; oferliorces V, 24 ;

zu den umlautlosen formen: oferleorad Prf. 4, 10; rehtas I, 23; vgl. S. § 384 anm. 3 und $\S 19,5$;

in allen formen ohne umlaut erscheint eawa: aedeaues II, 18; acdeauas XIV, 22; vgl. dazu § 29, III.

2) Praeteritum. Ursprünglich kurzsilbige. Die endung ist normalerweise -ede, -edon bei einfachem konsonanten am 
wurzelende und $i$-umlaut der wurzelsilbe. Beispiel: getrymedeI,32. Gemination des wurzelauslautenden konsonanten begegnet bei: Jerh-getrymmede I, 7; der mittelvokal ist synkopiert in bremmde $\mathrm{XI}, 33$; -ade findet sich in untrymade IV, 46; XI, 2.

Ursprünglich langsilbige. Nach langer silbe erscheint die endung des praeteritums mit synkope des mittelvokals $(\S 28, \mathrm{IV})$ als -de, das an die verba auf einfache liquida, oder einfachen nasal, auf $f,(s), g$, auf vokal oder diphthong $+d$ ohne weitere veränderung antritt. Der $i$-umlaut bleibt.

Beispiele: foerde Prf. 1,4; geherde Prf. 8, 7; geherdon I, 37; ingeberigde II, 9; owoerigdon IX, 28; S. $§ 405$ anm. 2; hremdon XI, 33; untynde Prf. 5, 10; woende Prf. 8,4; gelefde Prf. 4, 7; gedrygde XI, 2 (ohne synkope erscheint: gefylgede VI, 2; XVIII, 15); ahydde VIII, 59; tolaedde Prf. 3,9; vereinfachung des $d d$ zeigt sich bei gecîdon VI, 52 .

Die verba auf - $\delta$ lassen -de ebenfalls unverändert antreten: gecydde Prf. 1,5. Keine synkope findet statt bei den verben auf auslautende muta + liquida: froefredon XI,31; leðrede XI,2; ferner bei rocgetede 187,14 .

Ohne $i$-umlaut erscheint: uearmdon XVIII, 18 neben ucermde XVIII, 18. 25; vielleicht in vermischung mit wearmian.

Kleine veränderungen der normalform, die durch das zusammentreffen des $d$ der endung mit dem endkonsonanten der wurzelsilbe herbeigeführt werden, sind folgende:

Geminaten werden vereinfacht: ymbcerde Prf. 3,11; XXI,20; fylde Prf. 4, 15; gefyldon II, 7; spildic XVIII, 9; spildon XI, 53.

Die endung -de wird $\mathrm{zu}$-te nach den fortes $(p), t, c: g e$ moette, gemoetton I, 41;

vereinfachte schreibung findet sich bei gemoete II, 14; gemoetun I, 45; geondete Prf. 3,6; geondate I, 20;

etymologische schreibung erscheint bei togêc đe Prf. 5, 13; geneolecde Prf. 4, 13; geneolicde Prf. 3,12; S. $\$ 405$ anm. 10.

Nach konsonant $+d, t$ geht das $d$ der endung ganz verloren: sende I, 33; gesendon I, 19. 22; ymbgyrde XIII, 4; ymbuoende XX, 14 (zu wendan); gescyrte Prf. 3, 12, stylton XIII, 22; geoehton XV, 20; gircesti XXI, 20.

Ohne germanischen mittelvokal und infolgedessen umlautlos sind die praeterita folgender lang- und kurzsilbiger verba gebildet: geworhte Prf. 1,8; 7,2; worhton V, 29; auoehte Prf. 4,10; XII, 1; sohton V, 18; geðohton XI, 53; gebrohte Prf. 8, 5; 
gebrohton VII, 45; bebohton II, 16; tahte Prf. 8,12; XXI, 19; gelahte VIII, 20; gelahton XVIII, 12 ; rahte XII, 26 ; rahton XIX, 29; s. $§ 4$ b; salde IV, 5 ; XXI, 13; gesalde Prf. 3,$13 ; 7,1$; sealdon XIX, 3 ; s. § 16,2; acuoeldon XI, s3.

Analogisch ist der umlaut wieder eingeführt im praet. von setta: gesette Prf. 1,7; gesettes XX,15; setton XI, 34; gesetton VI, 10.

Die verba auf $-r w$ verlieren lautgesetzlich ihr $w$ vor dem ursprünglichen $i$ der praeteritalendung: smiride IX, 11.

Von den verben auf diphthong $+w$ ist eawa belegt mit durchführung des $w$ in allen formen: at-eaude Prf. 1,5; aedeude V, 20 (s. unter 1).

Von den verben auf ursprünglich -aw überträgt ceiga das $j(g)$ in alle formen: eftgeceigde Prf. 1, 3; geceigdon IX, 18 etc.

Ursprüngliches wurzelauslautendes $h$ ist geschwunden in dem verbum contractum: giðryde 188, 3; S. § 408 anm. 18.

Mit -ade nach klasse II sind gebildet: geoehtadon V, 16; inlichtade Prf. 6,1; ofergloesade 188, 7; geondetate IX, 22.

3) Part. praet. Ursprünglich kurzsilbige. Die endung ist -ed: getrymed Prf. 7, 4; gestyred Prf. 7, 4; gesettet Prf. 2, 2; ofergesetted XI, 38 mit analogischer gemination (s. unter 1); wegen $e t$, ed s. $\S 64, \mathrm{II}$.

Synkope des endungsvokals tritt in den flektierten formen auch vor vokalisch anlautender endung nicht ein: gesettedo II, $6 ; \mathrm{V}, 13 ; \mathrm{XX}, 5 ;$ gesattedo! XX, 6 ; asetedo XX, 6 ; getrymmedes IV, 39.

Ursprünglich langsilbige. Die endung ist -ed bei durchgeführtem $i$-umlaut: gebroeded XIX,13; gecyðed Prf. 1, 7; unascended Prf. 1, 12; acenned III, 4; gehoeled Prf. 4,6; aed-eaued Prf. 4, 4; cet-euwed XXI, 14 (s. unter 1); gefylled VI, 26; getimbred II, 20; genemned Prf. 3,9; ingemoetet Prf. 2,3; gegyrded XIII, 5; inlichtet Prf. 6, 2; -t für -d s. § 64, II.

In der flektierten form findet sich synkope des mittelvokals bei tyndo Prf. 8, 5; XX, 19; gedaclde XIX, 24; gebegdum XIX, 30; mit lautgesetzlicher vereinfachung der gemination bei ancenda I, 18; ancende III, 16; etymologische schreibung liegt vor in gefylldo VI, 12.

Keine synkope in der flektierten form haben gedroefedo Prf. 6, 1; gelceredo VI, 45; gecerredo Prit. 1, 10; gecearredo VII, 53; s. § 18 b; timbredes Prf. 6, 5; S. § 400 anm. 2. 3. 
Ohne germanischen mittelvokal sind gebildet die participia: aworht VI, 6 ; geworht.Prf. 3,2; gesoht Prf. 4, 17; ûnbocht $\mathrm{XV}, 25$. Diese bildungsweise ist übertragen auf gecnyht XI,44. S. $\S 407,2$.

Die endung -ad nach klasse II erscheint bei ad-eauad Prf. 3, 16 s. unter 1); eftgeboetad III, 4; awoergado VII, 49; geondetad Prf. $3,10$.

Die endung -en nach den starken verben erscheint bei ungelefen III, 36; XX, 27. S. § 406 anm. 7.

\section{$\S 75$. Klasse II.}

Ueber die praesensformen s. Kapitel XIII.

Praeteritum. Die regelmässige endung ist -ade, -adon: gelytlade Prf. 1, 9; gehalgade X, 36; gedofnade Prf. 1, 9; gefaestnade Prf. 4, 13; synngade IX, 2; gebiotate Prf. 7, 19; gewunade I, 39; fulguade III, 22; geondsuarade III, 10; geliornade VI, 45; S. § 416 anm. 12; ceapadon IV, 8; $u$-Umlaut hat: gismioðade 188, 4; gefeade III, 14; S. § 414 anm. 5c; tuiaton XIII, 22; S. § 414 anm. 5 b.

Mit -ede erscheinen: gewunede I, 32; gegeadredon Prf. 6,10; cunnedon VIII, 6; S. §411 anm.5; geondsuarede I, 50; -ode findet sich in fulgode I, 33; -ude begegnet bei trugude II, 24; ondsuearudon XIX, 7; s. S. § 413 anm. 6.

Der mittelvokal ist synkopiert bei ymbsceaude I, 42; sceode $\mathrm{XX}, 11$; untrymigdon VI, 2 und bei geondwearde Prf. 5, 13; I, 26; geonduarde VII,21; geonduorde XIV,23 etc., das ganz zu klasse I übergetreten ist.

Part. praet. Die regelmässige endung ist -ad: gelaðad Prf. 1,$9 ;$ gedaefnad Prf. 2, 4; geworठad Prf. 6, 2; gewintrad XXI, 18; getrahtad I, 38; gifead XIV, 28; -ad begegnet in gewidloed XVIII, 28; -ed in geuuldred XI, 4.

Flektierte Form ist gesommadum Prf. 2, 5.

\section{§ 76. Klasse III.}

Von den zu dieser klasse gehörigen verben sind folgende belegt:

1) habba. Praes. ind. 1. sg. haefic IV, 17; hafu, nafu IV, 17; hafo IV, 32; X, 18; 2. sg. hoefis IV, 11; hoefes IV, 18; VIII, 48; 3. sg. haefed III, 15; XIV, 21; hwefes III, 29; haefis IV, 44; ncefis 
$\mathrm{XIV}, 30$; pl. nabbo ue XIX, 15; habbad VIII, 41 ; gihabbad $\mathrm{XX}, 23$; nabbað II, 3; habað Prf. 7, 17; XVI, 22; habbas V, 38; $\mathrm{X}, 10$; XXI,5; nabbas XV, 22; habbbas XII, 35; opt. hoebbe X,10; $\mathrm{XII}, 36$; 188, 10; part. haebbende V, 5; habblend X, 21; haebende Prf. 4, 8; inf. habbe? V, 39; habbanne V, 26; praet. hafde Prf. 6,5 ; hafdest IV, 18; hafdestu XIX, 11; nafdes XIX, 11; nafdon $\mathrm{XV}, 22$; ncefdo IX, 41.

2) saccga. Praes. 1. sg. sago III, 3; saggcgo XVI, 25; 3. sg. gesageð Prf. 3, 6; sages Prf. 4, 11; pl. sagces XVIII, 29; imp. sag $\mathrm{XX}, 17$; inf. sagcganne XVI, 12; praet. scegdo III, 28; sagde I, 15; foresagde Prf. 6, 4; scegdest IV, 18; sagdon IV, 51; $\mathrm{XI}, 46$; part. praet. ascegd XXI, 25; gescegd Prf. 3, 3 .

3) lifiga. Praes. 1. sg. liofo VI, 57; XIV, 19; 3. sg. liofad Prf. 4, 7; XI, 26; liofaed XI, 25; liofed VI, 57; lifed VI, 51; lifes IV, 50; pl. lifias V, 25; XIV, 19; part. unlifiendum Prf. 4,20; lifiende VI, 51; lifigiendo VII, 38.

4) gefiaga. Praes. 3. sg. gefiið XV, 18. 19. $23\left({ }^{2}\right)$; gefied III, 20; gefiad VII, 7; XII, 25; XV, 23; inf. gefiage VII, 7; praet. gefiadon XV, 24 .

5) smeage. Praes. pl. smeas V, 39; inf. smeage VII, 52.

6) Jreaga. Praes. 3. sg. geđreað VIII, 46; XVI, 8.

7) giwiga. Praes. ind. 2. sg. giues IV, 9; gegiuad XI, 22; pl. giuað XVI, 26; gegiuað XIV, 14; gegiauad Prf. 7, 6; imp. giuad XVI, 24; giuas XV, 7; praet. gegiuuedes IV, 10; gegiuade XVI, 24; S. § 416 anm. 14 b.

8) forhycga. Praes. 3. sg. forhycgad V, 45; inf. forhygcanne Prf. 7, 12; part. forhycgende V, 45.

9) Vereinzelte belege sind: onscynað XIV, 27; bues I, 38; gebyde I, 14. S. § 416 anm. $11 \mathrm{c}, \mathrm{d}$.

Kapitel XVI. Kleinere gruppen.

$\S 77$. Verba praeteritopraesentia.

Erste ablautsreihe. 1) Praes. ind. 1. 3. sg. wat VII, 15.27; VIII, 55; nat VIII, 25; nât XII, 35; 2. sg. uast III, 8; naast XIII, 7; nastu III, 8; nast III, 10; pl. uuton III, 2; VII, 27; IX, 31; nutton IV, 22; nuuton IX, 29. 30; uutun ue XIV, 31; nuntu we XIV,5; untto VIII, 14; nuto ue XVI,18; nuutto VIII, 19; 
DIE SPRACHE DER NORTHUMBK. IN'TERLINEARVERSION.

untu IX, 21; opt. pl. wita XIII, 17; imp. uutasgie XV, 18; inf. gevuta XIT, 5; praet. 1. 3. sg. wiste Prf. 1, 14; XI, 42; nyste I, 31; uiste II, 24; 2.sg. wistes I, 48; pl. uiston VIII, 19; geuiston II, 9 ; nyston X, 5; uistun XXI, 12 .

2) Praes. opt. 3. sg. hage XIX, 24.

Dritte ablautsreihe. 1) Praes. ind. 1. 3. sg. conn VIII, 55; $\mathrm{XV}, 15$; pl. cunnon I, 26; praet. 1. 3. sg. cuðe I, 31; XVII, 25; сиðе 188, 3; 2. sg. cuðеs I, 48; pl. cuðon X, 5.

2) Praes. ind. 3. sg. ðorfä XIII, 10; part. ðorfendım XII, 5; jorfondo XII, 8.

3) Praet. s. sg. darste $\mathrm{XXI}, 12$.

Vierte ablautsreihe. Praes. ind. 3. sg. gemynes XVI, 21; S. $§ 423$ anm. 2; opt. pl. gemynan XVI, 4; imp. gemynasgie $\mathrm{XV}, 20$.

Fünfte ablautsreihe. Praesens ind. 1. 3. sg. mag V, 19.30; mage VI, 44; mago XIII, 37; pl. magon Prf. 7, 10; III, 9; mago V, 44; mago XIV,5; XVI, 12; opt. sg. mage Prf. 3, 15; I, 46; magi XXI, 25; pl. mago VII, 34; VIII, 6; mago XVII, 24; magon XIII, 36; inf. mcege Prf. 7, 9; praet. moehton VIII, 6; maehtun Prf. 5, 5 .

$\S 78$. Das verbum substantivum.

Wurzel $c s$ und or: Praes. ind. 1. sg. am Prf. 1, 13; 4,16; XII, 26; nam XII, 49; XVI, 32; XVII, 11; 2. sg. ard I, 19, 21; XVIII, 17 ; arst I, 19; 3. sg. is Prf. 1,$2 ; 4,5 ; 6,3$; nis VIII, 44; XV,20; pl. aron Prf. 1,10; 3,16; naron XVII, 14; arogie XIII, 11; narogie X, 26; aru VIII, 41; arun XXI, 16 rd.; sint Prf. 5, 8; I, 2; XV, 5; sinclon Prf. 6, 7; VIII, 33; opt. sg. sie Prf. 3, 4. 14; 4, 1; 6, 4; pl. se Prf. 3, 3; XII, 40; sê XII, 36; XIV, 1 ; sic III, 20; V, 34; XVII, 19; XXI, 25.

Wurzel bheu: Praes. ind. 1.sg. bium VII, 34; biom XII, 32; beom VIII, 55; XII, 26; XVII, 24; 2. sg. bist I, 42; 3. sg. bið Prf. 2, 5. 6; 3, 10; 6, 12; pl. biðon Prf. 5,4.12; 8, 8; VI, 45; biðo XVII, 6.

Wurzel wes: Praes. inf. wosa I, 12. 46; IX, 27; uôsa XX,27; imp. uosað XVI, 4; praet. ind. 3. sg. waes Prf. 1, 5; 2, 2. 3; 3,2.11; uass IX, 25; noess XI, 15; pl. woeron Prf. 1, 9; XII, 6; XV, 27; weron Prf. 1,$14 ; 3,2$; I, 24; uerun Prf. 3,8 ; uaron XXI, 8; neerun XXI, 8; opt. sg. woere Prf. 1,$6 ; 3,3.16 ; 5,11 ; \mathrm{XV}, 19$;

Anglia. N. F. XII. 
were Prf. 1,$7 ; 4,19 ; 5,13 ; 6,3$; ueri XIX, 28; XXI, 7; nere $\mathrm{XXI}, 23$; nêre XVIII, 30; nềre XI, 32; ucere XX, 15; pl. uoero $\mathrm{XIX}, 31$; woere $\mathrm{XV}, 19$; wero Prf. 5,15; uero I, 12; nerce XII, 42 .

\section{$\S 79$. Das verbum wollen.}

Praes. ind. 1. sg. uillo XVI, 25. 26; XVII, 24; XXI, 22; ucello XIII, 26; 2. sg. uiltu V, 6; nuilt XXI,18; 3. sg. wil III, 8; V, 21; pl. uallad Prf. 4, 15; VI, 67; VIII, 24; IX, 27; XII, 21; XVIII, 39; uallas VIII, 44; IX, 27; XXI, 3; uallon XVII, 20; nallas V, 40; opt. sg. uaelle II, 4 rd.; VII, 17; pl. uselle XV, 7, 10; imp. sg. noelle V, 14; VIII, 11; pl. nallað II, 16; VI, 43; nallas $\mathrm{X}, 37$; noellað VI, 20; nalle $\mathrm{V}, 45$; ncelle VII, 24; praet. 1.3. sg. ind. ualde Prf. 6, 3. 9; I, 43; XV, 19; 2. sg. ualdes XXI, 18 (3); pl. waldon V, 35; VI, 11; XVI, 19; opt. sg. ualde IV, 10; VII, 17; pl. ualde VIII, 42 ; nalde XVIII, 30 .

\section{$\S$ 80. Das verbum tun.}

Praes. ind. 1. sg. doam Prf. 7,6; XIV,14; XVII, 26; dôam XIV, 13. 31; doom XIII, 7; doem XI, 41; 2. sg. doas III, 2; dooes VI, 30; does VII, 3; 3. sg. misdoed III, 20; pl. doas XIII, 15; $\mathrm{XV}, 21$; doaठ Prf. 7, 1; XVI, 3; opt. sg. doe IV, 34; VI, 38; XVII, 26; undoe I, 27; gedoe XVII, 4; pl. dôe XI, 47; imp. sg. do XII, 27. 28; XX, 27 (2); dô XIII, 27; pl. doað II, 5; undoað XI, 44; undoeð II, 19; does VI, 10; part. praes. doende Prf. 4,9; inf. doa Prf. 4,15; undoce $\mathrm{X}, 35$; undoanne Prf. 3,13; praet. 1. sg. dyde IV, 29; V, 11; VII, 21; XIII, 12. 15; XV, 24 etc., pl. dydon V,29; dêdon VI, 23; opt. dyde XV,24; part. praet. gedoen VI, 6; undoen VII, 23.

\section{§ 81. Das verbum gehen.}

Praes. ind. 1. sg. gae VIII, 14; gae VIII, 22; XVI, 28; XXII, 3; gô XIV, 12; XVI, 16; gâae XVI, 5; 2. sg. gaces XIII, 36; 3. sg. gaað III, 8; gaeð VIII, 12; X, 4; ingaeð Prf. 6, 2; inngaed X, 2; gaas XI, 31; XII, 35; gegaas XI, 9; inngaas X, 1; opt. sg. ingae III, 4. 5; imp. gaa Prf. 4, 7; IX, 7; gâa XX, 17; gae VII, 3; gaea VIII, 11; inf. gegâa XVIII, 8; praet. 1. 3. sg. eade IV, 47.50; IX, 11; XVI, 28; geeade Prf. 4,16; eode IV, 43; VII, 1; IX,7; úuteode VIII, 59 ; pl. eadon IV, $30 ;$ geeadon VI, 66; eadun XVIII, 6; eodun XX, 10; XXI, 3. 


\title{
B) Nominalflexion.
}

Kapitel XVII. Deklination der substantiva.

\author{
Vokalische stämme. \\ $\S 82$. -o-deklination.
}

I. Masculina.

1) Nom. acc. sg. sind bei reinen $o$-stämmen normaler weise endungslos: lust Prf. 1, 3; Jreat Prf. 6, 12; иoeg Prf. 7, 4; I, 23; stream I, 28; gast Prf. 8,6; gaast VI, 63; hehstald Prf. 1,2.13; god I, 18; degn I, 43; waestem Prf. 2, 6; waestm XII, 24; finger $\mathrm{XX}, 25$; fingcer XX, 27; heofun I, 51; drihten Prf. 1, 9.

Mit -e erscheint doege VIII, 56; IX, 4; XIX, 31.

In vielen fällen ist ein $\bar{b}$ offenbar ganz mechanisch ohne irgend eine alterierung des geschlechts vor maskulina und auch vor feminina gesetzt; einige beispiele für beide fälle sind: $\not$ lust Prf. 1,$3 ; \not$ dreat Prf. 6,12 ; VII, 32; $\not$ gâst XIX,

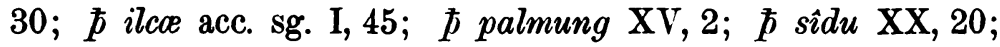
$\not \vec{b}$ bóc XXI, 25; sermoni-quem IV, 50 ist glossiert durch uord tzt done.

Von io-stämmen sind belegt kurzsilbige ohne endung: small XVIII, 22; lehnwort pytt IV, 11. 12; langsilbige und kurzsilbige auf $r$ mit der endung -e: esne XVIII, 10; VIII, 35; ende Prf. 1,12; hiorde Prf. 6,3; X, 11; bóecere XIX, 37 rd.; sceacere, settere X, 1; worðare IX, 31; godspellere Prf. 1, 2; here XII, 18; auf - $a$ geht aus hiorda Prf. 6,$4 ; \mathrm{X}, 11$; endungslos ist das lehnwort caser XIX, 15; uno-stämme sind: ðea Prf. 5, 16; オeau XIX, 40; laruu I, 38; laruû XX, 16; laruua XI, 8; XIII, 14.

2) Gen. sg. Die endung ist normaler weise -es: godes Prf. 1, 2; goddes Prf. 3, 4; middan geardes Prf. 3, 7; domes V,29; gastes 187,15 ; receles XIX, 39; drihtnes V, 4 ; S. $§ 244,1$; iostämme: hucetes Prf. 6,15 (ccesares XIX, 12 ist halblateinisch und nicht sicher als įo-stamm zu erweisen).

3) Dat. sg. Die endung des dativs bezw. instrumentals ist $-e$; doch sind daneben unflektierte formen nicht selten; je ein beispiel findet sich für - $\infty$ und -i: (Joer!) Jreate VII, 31 ; gode Prf. 1,2; dogge II, 23; streame III,26; gaste Prf. 3,7; degne Prf. 3,14; heofne III, 13; vgl. L. § 245; mérne I, 43 (instr. S. § 237 anm. 2); uarde XXI, 4; drihtne VI,23; oedle IV,44; S. §244,1; hiorode 188, 8 . 
Ohne endung kommen vor: lust Prf. 2,5; doeg Prf. 4, 19; Jreat Prf. 5, 6; geong IV, 6; lehtun XIX, 41; fredem I, 18; - - hat: duruuardae XVIII, 16; -i: doegi XXI, 14; s. vorwort; jo-stämme sind ende II, 10 rd.; esne XVIII, 10; ohne endung: caser XIX, 12.

4) Nom. acc. pl. Die endung ist -as, daneben vereinzelt -es und -o; S. § 237 anm. 3. -as: dagas Prf. 6,11; Jegnas Prf. 7,3 ; deafas X, 8; sestrus II, 6 ; hlafas VI, 9; stanas VIII, 59; stafas VII, 15; godas X, 35; -es: fisces VI, 9; lehnwort engles $\mathrm{XX}, 12 ;-0$ : hlafo VI, 5. 11; aldro IV, 20; VI, 31; IX, 18; goddo $\mathbf{X}, 34$ wenn es nicht als neutrum gebraucht ist; s. Braune, got. gr. $\S 94$ anm.3; -e findet sich in sestre II,6 rd. jo-stämme -as: setteras X, 8; smoellas XIX, 3; -as: esnæes IV, Ј1; êsnas XVIII, 18; -es: uorðares IV, 23; S. § 248; -o: lehnwort mynetro II, 14; uo-stämme: laruas VI, 45; celaruuas IV, 1 ; laruu? Prf. 2, 6 .

5) Gen. pl. Die endung ist - $a$; daneben vereinzelt -ana.

Beispiele: degna XX, 30; fisca XXI, 8; lehnworte: penninua VI, 7; ambilita XX, 30; - $e_{\$}$ in canone XIX, 30 rd.; -una: fiscana Prf. 8, 9; XXI, 6; dugana XX, 1. 19; io-stämme: mynetra II, 15.

6) Dat.pl. Die endung ist -un : dagum Prf. 8,6; fiscum XXI, 10; rapum II, 15; stafum V, 47; lehnwort: ambehtum XXI, 14; io-stamm: êsnum XVIII, 26; uo-stamm: celaruum VII, 48.

\section{Neutra.}

1) Nom. acc. sg. Reine $o$-stämme erscheinen ohne endung.

Beispiele: leht Prf. 1, 6; hus Prf. 4, 7; lif I, 4; lehtfoet V, 35; word Prf. 1, 6; bearn Prf. 3,9; godspell Prf. 2, 4; lehnwort: win Prf. 1, 9; 3,11; scipp VI, 17; lîn XIII, 4; tacon, becon Prf. 1, 7; waeter Prf. 3, 10; wundar II, 4 rd.; jo-stämme: gedd X, 6; cynn XI, 48; nett XXI, 6; stycg XIX, 23; rîc Prf. 3,15; êrn XVIII, 28; uoede XIX, 5; uoedo XIX, 24; heig VI, 10; S. § 247 anm. 3; uo-stamm: uuitreo XV, 1 ; treo $\mathrm{XV}, 2$ rd.

2) Gen. sg. Die endung ist -es.

Beispiele: uifes Prf. 1, 3; uordes Prf. 1, 6; uinnes Prf. 2,6; huses II, 17; writtes Prf. 7, 3; uotres V, 3 etc.; io-stamm: cynnes XVIII, 12. formen.

3) Dat. sg. Die endung ist $-e$, daneben viele unflektierte

Beispiele: -e: uife Prf. 4,4; uorde, uuritte II, 22; londe VI, 23; lehnwort uîne IV, 46; uatre I, 31; heafde XIX, 30; haefde XIX, 2; 
forleigere VIII, 41; ohne endung: calond Prf. 1, 11; leht I, 7; scipp VI, 19; hus VIII, 35; foex XI, 2; jo-stämme: uoede XIX, 2; cynn XI, 52; uoestern Prf. 3, 5; ucestern VI, 31. 49 - ist das northumbrische $r$ dieses suffixes vielleicht durch eine kontamination mit suffix -āri entstanden? vgl. carcern zu ahd karliâri, wenn hier nicht anlehnung an oern vorliegt.

4) Nom. acc. pl. Die endung ist $-o$, veinzelt $-a$ und findet sich bei den kurzsilbigen und meist auch den langsilbigen stämmen.

Beispiele: -o: fatto II, 6 ; tuiggo XV,5; tuicgo XII, 13 ; bibodo Prf. 7, 6; bebodo XIV, 21; wriotto V, 39; scioppo VI, 23. 24; uoerco III, 20; wordo Prf. 5, 2; gero V, 5; scipo Prf. 8, 11; scipo $\mathrm{X}, 3$; gihrîno 188,4 ; -a: beboda XV, 10 ; tuigga XV, 6 ; uorda XII, 47; uôrda XIX, 13; uerca V, 36; XIV, 10; uoerca VII, 7; $\mathrm{V}, 20$; ohne endung erscheinen die langsilbigen: uord $\mathrm{X}, 19$; XII, 50; uôrd X, 21; uoerc Prf. 3, 16; scîp Prf. 6, 4; X, 3. 4; scip $\mathrm{X}, 4$. 8; lond IV, 35; uuscbearn XIII, 33 und das kurzsilbige bebod XIV, 15.

Ursprünglich dreisilbige mit langer wurzelsilbe behalten die endung wie im ws. so: neteno IV, 12 ; ebenso erscheinen ursprünglich zweisilbige alle mit $-o,-a$ zum teil mit übernahme des sprossvokals aus den flexionslosen formen: ucetro III, 23; hraglo XX, 5 ; becno II, 23; uundra Prf. 8,1; IV, 48; taceno III, 2 ; beceno IV, 48 ; becena XII, 37;

io-stamm: uoedo XIII, 4; XIX, 23.

5) Gen. pl. Die endung ist $-a$, vereinzelt -ana, einmal findet sich 0 .

Beispiele: -a: scipa Prf. 6, 3; lehnwort pûnda XIX, 39; -ana: wriottana Prf. 2, 4; -o: taceno Prf. 2, 1.

6) Dat. pl. Die endung ist -um: brydlopum Prf. 1,3; blodum I, 13; uordum V, 47; hrceglum XIX, 40 etc; uo-stamm: treum XIX, 39.

\section{$\S 83 . \quad \bar{a}$-deklination.}

Ueber die flexion der feminina dieser klasse vgl. besonders: Lindelöf, Beiträge zur kenntnis d. altnorthumbrischen: Mémoires de la Société Néo - Philologique à Helsingfors I 245-284. Ihr hauptkennzeichen ist der ausgang des gen. sg. auf -es; die gelegentliche endungslosigkeit des acc. sg. und der ausgang der $i \bar{a}$-stämme auf $-e$ bei denen auf $-n i s(e)$. 
1) Nom. sg. Die endung der kurzsilbigen ist -o belegt in: gefo I, 17; lufo XVII, 26. Die langsilbigen erscheinen gewöhnlich ohne endung: feber-adol IV, 52; ned-ðarf Prf. 3, 12; lar Prf. 2,6 ; stefn Prf. 3,5; elnung II, 17; halgung X, 22; huosstrung VII, 12.

Regelmässig erscheint die endung in denen auf $-i \not \bar{o} ; \mathrm{S}$. $\S 255,3$, s0: «ræðठठо III, 36 .

Ausserdem findet sich der ausgang -e bei stefne I, 23; - $a$ bei farma XIII, 2; -i im lehnwort segni XXI, 11, s. vorwort; iastämme ohne endung: byrgenn XIX,41.42; synn IX, 41 ; sibb XX, 19; ûnsib VII, 43; Jignen XVIII, 17; an lehnwort Jîr XVIII, 17.

Von denen auf -nis erscheinen ohne endungs-e nur cyðnis VIII,14; XXI, 24; at-eaunis Prf. 1, 1; mit -a uittnesa VIII, 17; alle übrigen mit $-e$ s. Lindelöf a.a. 0. p. 275; uāstamm stou IV, 20 ; stoue XIX, 20.

2) Acc. sg. Bei den kurzsilbigen finden sich die endungen $-e,-a,-\imath,-o$ belegt in: ondsucere I, 22; ondsuare XIX, 9; geafa Prf. 3, 4; lufu Prf. 7, 10; lufo XV, 13.

Bei den langsilbigen erscheint der acc. sg. endungslos regelmässig in denen auf -ung mit ausnahme von fiunge Prf. 7, 12 s. Lindelöf a. a. 0. p. 260. Die übrigen belege ohne endung sind: eorlippric XVIII, 26; beer V, 7; lâr XVIII, 14; huil V, 6; [ða huile V, 7 s. Lindelöf p. 253]; run Prf. 3, 14; tîd Prf. 4, 11; saucl Prf. 6, 3; saul XIII, 37; stefn III, 8; sceaed XVIII, 11.

Die endung -e weisen auf: lare XVIII, 19; saule 188, 9; bêre Prf. 4, 8; bisene Prf. 7,1; halle XVIII,28; aare IV,44; inlâde 188,9 ; stefne V, 37; cestre IV, 5 ; ae ist endungsvokal bei milsae $188,11$.

Auf -a gehen aus: mearda IV, 36; earlipprica XVIII, 10; farma XII, 2; roda XIX, 17.31; die lehnworte ceastra XI, 30. 54; segna XXI,6; auf -o: farmo XXI, 20; иræððo Prf. 4,3; S. § 255,3; auf $-i$ : lehnwort sêgni XXI, 8 s. vorwort. $i \bar{a}$-stämme. Keine endung findet sich bei sib Prf. 7, 8; sibb Prf. 8, 5; synn VIII, 34 (synne geändert zu synn) IX, 41; -e hat synne Prf. 5, 16; sowie die auf -nis; - $a$ in uitnessa III, 11; uitnesa VIII, 18 s. Lindelöf p. 276: uña-stämme: stoue XI,48; XIX, 17; stouce XIV ,2; stôu XIV,3.

3) Gen. sg. Die normale endung ist -es: rodes Prf. 8,12; leasunges Prf. 5, 16; Jrouunges Prf. 7, 19; capinces II, 16 ; $j \bar{a}$ stämme: synnes Prf. 5, 16; VIII, 34. 
Die auf -nis schwanken in der endung zwischen -es und -e. -cs: unrotnises Prf. 7, 15; selenises Prf. 7, 19; soðfoestnisses 1,14 ; geliorneses II, 1; -e: geliornisse Prf. 1, 14; smirinese XII, 3; settnesse Prf. 6, 5; hefignise XVI, 21 s. L. p. 278, 279.

4) Dat. sg. Bei den kurzsilbigen erscheinen die endungen $-a,-o,-u$ belegt in: geafa 187,15 ; lufa XV, 10 rd.; lufo XV, 10; luf $\iota \mathrm{XV}, 9$.

Bei den langsilbigen ist die gewöhnliche endung -e.

Beispiele; adle V, 4; deade IV, 43. 45; lare XVIII, 19; rode Prf. 1, 4; stefne Prf. 4, 6; uommbe III, 4; -a findet sich bei : forosaga 187, 14; farma Prf. 6,19; lehnwort ceastra XI, 1 ; $-o$ in den abstracta auf $-i \not \bar{o}$ : laøðठo XV,18; gesihðo Prf. 8,3 [efterðare $=$ post visionem $]$.

Ohne endung erscheinen: laar VII, 17; sprêc IV, 42; half XXI, 6; meigð Prf. 1, 11.

Schwanken herrscht bei denen auf -ung.

Die endung - $e$ begegnet bei: ceigunge Prf. 6, 8; Jrounge Prf. 8, 12; hearnunge Prf. 7, 10; fiunge XV, 18; insceaunge Prf. 8, 7; endungslose formen sind: throuung Prf. 7, 19; somnung IX, 22; ebolsong X, 33; racing Prf. 7, 3; racing Prf. 8, 11. $i \bar{a}$-stämme. Die endung ist $-e$ : synne Prf. 5,12 ; byrgenne $\mathrm{XX}, 1$; gerde XIX, 29; zu byrgenn XX, 1 und synno VIII, 21; XV, 22 s. L. p. 272 ; unclannise Prf. 2,3 ; sodfastnise V, 33; êcnisse Pif. 5, 18.

Der ausgang - $a$ findet sich in onuitnesa $\mathrm{XX}, 30$; $-i$ in deigilnisi 187, 14 s. vorwort; zu soðfaestnis XVIII, 37 s. Lindelöf p. 277;

uña-stämme: stoue III, 23; VI, 23; stou V, 13.

5) Nom. acc. pl. Als endungsvokale erscheinen $-o,-a,-e$.

Beispiele: -o: bydno II, 6 ; farmo II, 1; laro VII, 38; elno XXI,8; -a: worulda 187,10; mercunga Prf. 3,1 ; earnunga 188,12; screadunga VI, 12 ; -e: 才oncunge VI, $11 ; \mathrm{XI}, 41 ; i \bar{a}$-stämme: synna XX, 23; synno Prf. 3,6; aed-eaunise Prf. 2, 1; uä-stämme: lesua $\mathrm{X}, 9$.

6) Gen.pl. Die endungen sind -ana und -a.

Beispiele: farmana Prf. 1, 9; reordana Prf. 7, 10; sighðana Prf. 1,11; farma Prf. 3, 12; screadunga VI, 13; gearuunga XIX, 31.

7) Dat. pl. Die endung ist -um. 
Beispiele: fermum Prf. 3, 10; farmum II, 2; somnungum XVI, 2; i $\bar{a}$-stämme: synnum Prf. 1,$7 ;$ byrgennum V, 28; heannissum VIII, 23.

\section{$\S 84 . \quad i$-deklination.}

I. Masculina.

1) Nom. acc. sg. Kurzsilbig olne endung ist styd XIV, 2; XIX, 17; S. § 263 anm. 5 ; mett IV, 32; met IV, 58; megulit V, 38; uorðscip IV, 44; gelefenscip XV,22; auf -o: soðcuido X, 6 .

Langsilbige: erest Prf. 3,13 rd.; XI, 25; sa VI,1. 18; \$. §§ 266 anm. 1, 269 anm. 4; ymbcerr V,3; dael IX, 23; dynt XVIII, 22.

Eigentiimlich ist afista 1I, 17; dessen ausgang vielleicht eine unwillkürliche anlehnung an das superlativsuffix erfahren hat.

2) Gen. sg.: eristes Prf. 8, 1; metes XIX, 14.

3) Dat. sg. Die endung -e haben: argscipe Prf. 5,8; styde $\mathrm{XI}, 6$; neutr. spere XIX, 34 .

Unflektiert sind: styd VI, 10; XIX,41; derne-legerscip VIII,4; frumsceaft Prf. 1, 12; feng Prf. 8, 9; stenc XII, 3; crist XI, 24; sae Prf. 4, 16; sae Prf. 8, 8 s. S. § 266 anm. 2.

4) Nom. acc. pl. Von den kurzsilbigen haben die endung -o: cuido XIX, 30 rd.; metto IV, 8; S. § 263 anm. 3; auf -o gehen auch aus die langsilbigen: dêelo XIX, 23; spyrdo VI, 19; auf -as: dyntas XIX, 3.

5) Gen. pl. Die endung ist - $a$ belegt in lieda Prf. 4, 5.

6) Dat. pl. Die endung ist -um: belegt in liodum II, 23; spyrdum XI, 18; daclum 188, 7; soðcuidum XVI, 25.

\section{Feminina.}

Ueber die flexion der $i$-feminina s. L. a. a. $0.284-289$.

1) Nom. sg. ist endungslos: $a \mathrm{I}, 17$; insiht Prf. 1, 1; onsion XI, 44; sed VIII, 33; uoen IV, 10.

2) Acc. sg. Neben den endungslosen formen dêd Prf. 1,6; a VII, 19; XIX, 7; maht Prf. 6, 5; XVII, 2 erscheinen mit -o brydo III, 29; mit -e onsione VII, 24.

3) Gen. sg. losuistes XVII, 12.

4) Dat. sg. Endungslos sind: maeht 187, 15; sêd VII, 42; voyrd Prf. 5, 10; auf $-e$ geht aus onsione XX, 30 . 
DIE SPRACHE DER NORTHUMBR. INTERLINEARVERSION.

5) Nom. pl. Die endung ist -0 .

Beispiele: dedo Prf. 8,1: ûndedo III, 19; über glôedi XXI 9 s. vorwort; die ursprüngliche endung - Lindelöf p. 287 - liegt. darin keinesfalls vor.

6) Gen. pl. wyrtana XIX, 39.

7) Dat. pl. gloedum XVIII, 18.

\section{$\S 85$. $u$-deklination.}

I. Masculina.

1) Nom. acc. sg. Kurzsilbige mit erhaltener endung: sunn Prf. 3,$10 ; \mathrm{I}, 45 ; \mathrm{XIX}, 7$; sune I, 18; IV, 47; V, 19 ; XVII, 1 ; suna XIII, 31; neutr. feolo VI, 11; XIV, 30 etc; feh 188, $5 \mathrm{~s}$. S. $\S 275$.

Langsilbige: deað Prf. 5, 17.

2) Gen. sg. Kurzsilbige: sunu III, 18; sunce VI, 53.

Langsilbige: deaðes Prf. 2, 3.

3) Dat. sg. sunu III, 36 ; sune IV, 5 .

Langsilbige: deaðe Prf. 4, 10; erstarrte formen in afra I, 18; naefra XIX, 41.

4) Nom. pl. sunu, oft geändert aus suno IV,12; XXI, 2; suno VIII, 37; XII, 36.

5) Acc. pl. Kurzsilbige suno Prf. 3,3; XI, 52.

Langsilbige: uintro II, 20.

6) Gen. pl. uintra Prf. 4, 8.

7) Dat. pl. đornum XIX, 2.

II. Feminina.

Ueber die flexion dieser feminina s. L. a. a. 0. p. 290.

1) Nom. sg. duru $X, 7.9$.

2) Acc. sg. Kurzsilbige: duru Prf. 6, 2; X, 1. 2.

Langsilbige: hond III, 35; hônd XX, 27 .

3) Dat. sg. Kurzsilbige: dure XVIII, 16; to duru? XVIII, 16, Langsilbige: earde 1T, 44; honde XVIII, 22; hond X, 28 .

1) Nom. pl. Kurzsilbige: duro Prf. 8, 5; XX, 19.

Langsilbige: hond XI, 44 s. L. p. 290, 291.

5) Acc. pl. hôndo XXI, 18; hônd XII, 9.

6) Gen. pl. honda Prf. 8,7.

7) Dat. pl. durum XX, 26; hondum Prf. 8,5; XX, 25. 


\section{Konsonantische stämme.}

$\S 86$. $n$-deklination.

\section{Masculina.}

Die obliquen kasus verlieren ihr ursprüngliches auslautendes $-n$ und es erscheint eine vokalische endung, die zwischen $-a(-a)$, $-e$ und $-o$ schwankt. Dies erklärt sich wohl daraus, dass diese endungsvokale nur noch mit murmelstimme gesprochen wurden, ähnlich wie im nhd.

1) Nom. sg. - $a$ : lichoma Prf. 1, 6; brydguma Prf. 4, 1; monnslaga Prf. 5, 16; noma 1, 6; witga I, 21; cofa XI,38; hera XII, 26; hona XIII, 38; burna XVIII, 1; ucella IV, 14; gefea III, 29; -a: uitgae IX, 17; geuunce XVIII, 39; lehnwort oncrae 188,4; -e: ucelle IV, 6; bydle IX, 31; morsceaðe XVIII, 40; -o: uillo VI, 39.

2) Acc. sg. -a: lichoma Prf. 5, 1; ucella IV, 12; brydguma II, 9; noma XII, 28; bolla XIX, 29; groefa XIX, 31; gefea I, 14; $\mathrm{XV}, 11$; -o: uillo IV, 34; VI, 38 ; -e: fole $(b)$ Prf. 6,13 ; endungslos: uoel V, 4; lichom VI, 54; hel XIII, 18.

3) Gen. sg. Hier stehen sich die endungen $-(e, a, a) s$ und -a gegenüber.

Beispiele: -es: lichomes Prf. 2,3; brydgumes III, 29; -as: lichomas XVII, 2; gefeas I, 14; -ces: lichomaes I, 13; -a: lichoma Prf. 3, 13 rd.; uitga XII, 38; giroefa XVIII, 28.

4) Dat. sg. -a: frumma Prf. 1,12; lichoma Prf. 5, 2; forma Prf. 3, 2; uitga Prf. 3,6; noma Prf. 7,6; gefea III, 29; -a: folae XII, 15; -e: uaelle Prf. 4, 4; uele IX, 7; groefe XIX, 21; -o: uillo I, 13. Die endung scheint bezeichnet in cempan (geschr. cempā) XIX, 23.

5) Nom. pl. -a: uitga VIII,52. 53; -o: cempo XVIII,12; XIX, 23; lichomo XIX, 31; crendureco XX, 12 rd.; zu sciu XIX, 31; vgl. S. § 277 anm. 2.

6) Acc. pl. uillo VIII, 44; exin II, 14. 15; sciu XIX, 32; lehnwort ora 188, 9 .

7) Gen. pl. cempa XIX, 34.

8) Dat. pl. uitgum I, 45; gefoerum XI, 16.

\section{Feminina.}

Ueber die flexion der schwachen feminina s. L. a. a. 0. $290-295$. 
DIE SPRACHE DER NORTHUMBR. INTERLINEARVERSION. 91

1) Nom. sg. a: hearta Prf. 7, 4; heorta XIV,27; S. § 280; sunopa II, 15; lehnwort moessa VII, 2; e: paccille V, 35 .

Adjektiv-abstracta got. auf $-e i$, ws. auf $-u$ haben -0 : hoelo IV, 22; menigo V, 3.

2) Acc. sg. e: ðacccille Prf. 4,12; eorðe XXI, 11; a: hearta XII,40; ae: culfrae I, 32; nêdra III,14; u: eorðu III,22; XVIII,6; sîdu XIX, 34; XX, 20.

Adjektiv-abstracta haben -o: fyrhto VII, 13; aldo IX, 21; giðyngo, snyttro 188, 11; ebenso eorðo XVII, 4.

3) Gen. sg. Lehnworte: cirica X,22; hefid-ponna XIX, 17.

4) Dat. sg. a: uisa XIV, 11; heorta XIII, 2; hearta XII, 40; $e$ : eorðe VI, 21 (ðoem); eorðe VIII, 6; o: eorðo III, 31; earðo XII, 24 ; sido XX,27; in den adjektiv-abstrakten: unhoelo $\mathrm{V}, 4$; menigo VII, 31; u: eorðu VIII, 8; IX, 6; sîdu XX, 20. 25.

5) Nom. pl. adj.-abstr. Jiostro I, 5 .

6) Acc. pl. culufro II, 14; culfero II, 16; Jiostro III, 19.

7) Gen. pl. sunna XX, 1.

8) Dat. pl. Jiostrum I, 5; sidum Prf. 8, 7; uisum Prf. 7, 10.

\section{Neutra.}

1) Nom. sg. eastro Prf. 3,$12 ; \mathrm{XI}, 55 ;$ S. $§ 278$ anm. 3.

2) Acc. sg. eostro XVIII, 28.

3) Gen. sg. eastres Prf. 6,11; eostres XII, 1 .

4) Dat. sg. costro II, 23; eastro VI, 4; eastrae XI, 55.

5) Nom. acc. pl. ego IV, 35; IX, 6. 9.

6) Dat. pl. egum XI, 41; êgum XII, 40.

$\S 87$. Wurzelstämme.

I. Masculina. Nom. sg. mon II, 10; aldormonn II, 9; acc. sg. monnu VII, 22; IX, 1; monno Prf. 4, 7; VII, 23; S. § 281 anm. 1; gen. sg. monnes I, 51; dat. sg. menn II, 25; aldormen II, 8; nom. pl. menn III, 19; moneðo IV, 35; foet Prf. 6,11 ; feoðor-fota IV, 12 adj.? acc. pl. aldormenn VII, 32; heremenn XVIII, 3; foet Prf. 6,19; fôet XI, 2; gen. pl. monna I, 4 ; XII, 43; monno Prf. 4, 15; dat. pl. monnum IV, 28; nonnnum V, 41; fotum XI, $32 ; \mathrm{XX}, 12$.

II. Feminina. Nom. sg. bôc XXI, 25; burug V, 2; noeht IX, 4; XIII, 30; acc. sg. boc Prf. 1,11 ; burug II, 12; dat. sg. boec Prf. 1,13; boêc XX, 30; byrig Prf. 2, 1; neeht III, 2; XXI, 3; acc. pl. bôec XXI, 25. 
$\S 88$. Stämme auf $-r$.

1) Ws. faeder; nom. sg. fader Prf. 4, 9; feder XVI, 27; acc. sg. fader Prf. 7, 9; XIV, 7; faeder V, 19; gen. sg. fadores I, 18; VI, 39; faderes X, 29; fador VI, 40; dat. sg. feder Prf. 4,7; VI, 65; fêder XX,17; faeder Prf. 7, 18; VIII, 38; fader VIII, 44; nom. pl. fadero VI, 31; dat. pl. fadrum Prf. 2, 2.

2) Ws. brōðor; nom. acc. sg. broðer I, 40. 41; XI, 19; broder Prf. 3,9; dat. sg. broeðer XI, 19; nom. pl. brođro II, 12; dat. pl. broðrum XXI, 23.

3) Ws. mōdor; nom. acc. sg. moder Prf. 1, 4; II, 1; gen. sg. moderes III, 4; XIX, 25; dat. sg. moder XIX, 26.

4) Ws. dohtor; nom. sg. dohtor XII, 15.

5) Ws. sweostor; acc. sg. suoester XI, 5; gen. sg. suoester $\mathrm{XI}, 1$; nom. pl. suoestro $\mathrm{XI}, 3$.

$\S$ 89. Stämme auf $-n t$.

Nom. sg. haelend Prf. 4, 5; londbuend XV,1; yfeldoend XVIII, 30 ; freond XI, 11; friond III, 29; XIX, 12; acc. sg. helend Prf. 4,1 ; londbucend Prf. 7, 9; helpend V, 7; gen. sg. hcelendes II, 3; XIII, 23; dat. sg. hoelend I, 42; uitend Prf. 2, 5; nom. pl. friondas $\mathrm{XV}, 14$; acc. pl. friondas $\mathrm{XV}, 15$; dat. pl. friondum $\mathrm{XV}, 13$.

$\S 90$. Stämme auf -es, -os.

Das suffixale -er, -or $(<-e z,-o z)$ ist meist geschwunden, hat aber zum teil $i$-umlaut hinterlassen; so im nom. sg. doeg Prf. 1, 14; symbeldoeg V, 1 ; dat. sg. doeg I, 39; doege IV, 45; sunnedoeg Prf. 4,9; das suffix in der stufe -or ist erhalten im acc. pl. lomboro XXI, 16; lombor XXI, 15.

Kapitel XVIII. Deklination der adjektiva.

$\S 91$. Starke flexion.

Ueber die schwierigkeit, die adjektiva nach genus und kasus genau zu bestimmen s. L. §168. In der regel ist für das glossierende ae. adjektiv gleiches genus und gleicher kasus vorauszusetzen wie für das unmittelbar darunter stehende lateinische adjektiv ohne rücksicht auf das zugehörige ae. substantiv.

1) Nom.sg.masc. ist endungslos bei: cuðXVIII,15; halig V,1; heofoncund VI, 31 rd.; leas VIII, 44; oder XV, 24; er geht auf -e. 
aus bei ane VIII, 9; adligne XI, 1; gladde XVI, 20; doch liegt es nahe, hier an schw. flexion zu denken.

jo-stämme: fremðe Prf. 2, 2; smoeðe XIX, 23; S. § 303 anm.2; gesêne Prf. 3,11; giseni $\mathrm{XX}, 20$; s. vorwort.

2) Nom. sg. neutr. erscheint durchweg endungslos bei reinen $o$-stämmen.

Beispiele: all Prf. 4,7; VI, 39; XI, 50; full I, 14; XIX, 29; sôঠ IV, 37; XXI, 24; stið VI, 60; an VII, 21; micil VI, 10.

jo-stamm: niuce XIX, 41.

3) Nom.sg. fem. Die endung ist -o bei den kurz- und mehrsilbigen, wie menigo XII, 9. 12 ; micelo $\mathrm{V}, 3$; part. praet. efnegesettedo $\mathrm{V}, 13$; die langsilbigen sind endungslos, so: all IV, 53; oðer I, 29; soð I, 9; tuufald Prf. 1,3.

una-stamm: gearua VII, 6 .

4) Acc. sg. masc. Die alte endung -ne findet sich bei berhtne $\mathrm{XII}, 28$; blindne IX,1; enne VIII, 41; oderne Prf. 4, 13; Jyrnenne $\mathrm{XIX}, 5$; auf $-e$ gehen aus die part. praes. hocbende Prf. 4,8; wyrcende $\mathrm{V}, 18$; s. S. $§ 305$ anm. 1 ; - $a$ ist endung bei ana VIII, 29; s. oben ane.

Unflektiert sind: dead Prf. 6, 8; halig Prf. 8, 6; gelic Prf. 4, 9; reht, soð VI, 32; all VII, 23; hal XII, 27; micil XII, 24.

jo-stämme: arwyrdlicne Prf. 6,16; fremðe $\mathrm{X}, 5$.

5) Acc. sg. neutr. ist durchweg endungslos: all XVII, 2; an IX, 25; X, 30; . cuð XVII, 26; felleread XIX, 5; long V, 6; cuic IV, 10 ; s. S. $\S 303$.

jo-stämme: êce Prf. 4, 3; lifuelle IV, 10; nina XIII, 34; part. praes. auf $e$ : loedende, niomende Prf. 3,6 .

6) Acc. sg. fem. endet auf $c, o$. Beispiele $e$ : lytle Prf. 7, 14; part.praet. begrippene Prf.5,8; o: part. praet. gestyredo Prf.6, 17 . $i \bar{a}$-stamm: cloen Prf. 3, 14 .

7) Gen.sg. masc. Die endung ist -es: aganes Prf. 3, 13 rd.; odres XIII, 14; XIX, 32; halges 187, 15; unflektiert erscheint eftgemyndig Prf. 7, 16.

8) Gen. sg. neutr. yfles V, 14.

īo-stämme: ece VI, 68 (unflektiert?); niuces Prf. 6, 5 .

9) Dat. sg. masc. Die alte endung -um erscheint bei halgum Prf. 5, 4; II,23; michum VI,18; oðrum XV,25; anum VIII,9. Auf $-e$ gehen aus: ane V, 44; halge Prf. $3, .7$; micle VII, 37; part. praet. gecuedne Prf. 1,13; auf -o: oðero II, 1 ohne synkope. 
10) Dat. sg. neutr. Auf -um gehen aus: godum $X, 33$; fellereadum XIX, 2; part. praet. gebegdum XIX, 30; auf -e geht aus degle VII, 4.

11) Dat. sg. fem. Die alte endung $-r e(-r a)$ erscheint bei oðre $\mathrm{X}, 1$; oðrae $\mathrm{XIV}, 11$; soðre Prf. 5,11 . Auf -e gehen aus: milce (verschrieben für micle) XI, 43; uutearde XVIII, 22 . Unflektiert ist monig XII, 6.

12) Nom. pl. masc. Die gewöhnliche endung ist -o. Beispiele: allo XVIII, 20; eadgo Prf. 8, 8; halo V, 34; soðo IV, 23; menigo VI, 9; monigo Prf. 4, 4; deado VI, 49; freo VIII, 36; ôðro $\mathrm{XX}, 25$; oðoro VII, 12 ohne synkope; blindo IX, 40; godo Prf. 4,11 ; eftgemyndigo II, 17; part. praes. und praet.: fylgendo Prf. 3,8; indrungno II, 10; gecerredo Prf. 1, 10. Auf -e gehen aus: alle I, 7 ; deade Prf. 5, 12; VIII, 53; ane VI, 22; syndrige II, 6 ; menige VII, 31; oðre XII, 29; part. praes. und praet.: cuoeðende XII, 21; geunrotsade XVI, 20. Auf -a gehen aus: deada VIII, 24; micla XXI, 11; auf $i$ : ođri XXI, $8 \mathrm{~s}$. vorwort. Unflektiert ist: ûntrymig VI, 2.

io-stämme: ondfengo VII, 39; clano XV, 3; clane XIII, 10.

13) Nom. pl. neutr. Die gewöhnliche endung ist -o. Beispiele: stonino II, 6; uncuðo III, 10; huito IV, 35; toueardo XVIII, 4; yflo VII, 7; ungelico Prf. 5, 4; odvo, menigo XXI, 25. Auf $-e$ gehen aus: alle Prf. 3, 2; I, 3; part. praet.: gewordne Prf. 3,16; auf -a: soða $\mathrm{X}, 41$. Unflektiert ist geinsetet Prf. 1, 10 .

jo-stamm: gecuoemo VIII, 29.

14) Nom. pl. fem. Die endung ist -o bei: menigo III, 23; oðero VI, 23 ohne synkope; part. praes. und praet.: lifigiendo VII, 38; geuordeno II, 1 ; tyndo Prf. 8, 5; XX, 19. Auf - $a$ endigt meniga XIV, 2.

15) Acc. pl. masc. Die endung ist $-o$ bei allo Prf. 3, 7; menigo IV, 1; deado Prf. 4, 10; oðro XIX, 18; frio Prf. 5, 15; S. § 297 anm. 2; part. praes. und praet. foendo Prf. 4, 13; forletno Prf. 7,17; - $e$ ist die endung bei alle Prf. 2,3; $-a$ bei freondleasa XIV, 18; -i bei syndrigi XXI, 25 s. vorwort.

16) Acc. pl. neutr. -o ist die endung bei godo $\mathrm{X}, 32$; menigo Prf. 5,6; VII, 31; X, 32; XIV, 30; [feolo XIV, 30]; ođro Prf. 5, 16; бa оðеro Prf. 5, 1; eorðlico III, 12; cuðigo XV, 15; part. praes. foedendo Prf. 8,$11 ;-a$ ist die endung bei goda 
V, 29; micla XII, 37; suindriga Prf. 2,5; cuða XV,15; soða XIX, 35; -e bei alle IV, 25; XIV, 26; XXI, 17. Unflektiert sind: agan I, 11; hagen XIX, 27.

17) Gen. pl. Die endung ist -ra bei allra Prf. 7, 1; XIX, 30 rd.; - $a$ bei blinda X, 21.

18) Dat. pl. Die endung ist -ım: aldum Prf. 1,9; allum Prf. 3, 7; oðrum Prf. 3, 10; miclum XXI, 11; syndrigum VIII, 44.

\section{$\S 92$. Schwache flexion.}

1) Nom. sg. masc. Die endung ist $-a$ bei đe ancenda $\mathrm{I}, 18$; Je gecorena IV, 25; uiðeruorda XIII, 27; unhala $\mathrm{V}, 7$; $-e$ ist die endung bei mare VIII, 53; soðfeeste XVII, 25; anfalde, ðrifalde 187, 10. - jo-stamm: đe broema XIX, 37 rd.

2) Nom. sg. neutr. Die endung ist -e bei wyrse V, 14; part. praet. be - (for) numene Prf. 1, 7; -a bei afterra IV, 54 .

3) Nom. sg. fem. teigða I, 39; sesta IV, 6.

4) Gen. sg. masc. ancende III, 18; ðces blindes XI, 37; бoes blindborenes IX, 32 .

5) Gen. sg. neutr. io-stamm diorwyrðe XII, 3.

6) Dat. sg. masc. neutr. - $e$ ist die endung bei $\delta c e m$ blinde IX, 17; ðxem hlotmeste Prf. 4,19; loetmeste XVI, 23; halge I, 33; part. praet. gedolfene Prf. 2, 1; -a bei бoem hloetmesta VI, 39; hloetmoesta VI, 44; Jridda XXI, 14; -ce bei hloetmoestoe VI, 54.

7) Dat. sg. fem. suiđre XXI, 6.

8) Acc. sg. masc. neutr. Die endung ist - $a$ bei Jone forma II, 10; mara Prf. 7, 2; I, 50; wurresta, uirresta II, 10 rd.; suidra XVIII, 10 ; -e bei part. praet. forcuoedne Prf. 7, 13; betre IV, 52; $\not$ ane $\mathrm{V}, 18$; mare XIX,11; done ungeleaffulle Prf. 4, 3; jo-stamm gesene Prf. 7,14 ; - $\infty$ bei ilca I, 45; IX, 21 ; -o bei Jone blindo Prf. 5, 19; arro XV, 18.

9) Acc. sg. fem. Die endung ist - $a$ bei mara XV, 13; -o bei maasto XV, 13 ; -e bei part. praet. 1 tobrocene XIII, 26.

10) Nom. pl. masc. neutr. Die endung ist -o bei $\partial a$ yflo Prf. 4,11; бa deado V,25; dailco X,6; XIX,15; formo XIX,32; - $a$ bei ða soðfoesta XXI, 16 rd.; ðailca V, 36; niua Prf. 1,10; -e bei alle Prf. $1,10$.

11) Acc. pl. masc. neutr. Die endung ist $-o$ bei $\delta a$ deado V, 21; ठa synnfullo IX, 31; бailco I, 38; X, 8; $а$ cependo part. 
praes. Prf. 3,$12 ; \delta a$ âgno X, 3; $a$ syndrigo $\mathrm{X}, 4 ;-a$ bei $\delta a$ maasta V, 20; ða mara XIV, 12 . Unflektiert erscheinen $\delta a$ linnin, lînnen $\mathrm{XX}, 5.6$.

12) Acc. pl. fem. ða ilca II, 7; XI, 19.

13) Gen. pl. Die endung ist -ra bei unhalra, adligra, haltra $\mathrm{V}, 3$; scryngcara, biddendra $\mathrm{V}, 3$; fremdra $\mathrm{X}, 5$; lioniandra, sittendra XIII, 28; rcestendra XXI, 12; -ena bei blindena V, 3; - $a$ bei utacunda $\mathrm{X}, 5$. Ueber hlingindi $\mathrm{XXI}, 12 \mathrm{~s}$. vorwort.

14) Dat. pl. Die endung ist -um. Beispiele: halgum 188,2; part. praes. und praet. redendum Prf. 1,8; soecendum Prf. 2,5 ; frasendum Prf. 3,4: gesonnadum Prf. 2,5. Unflektiert ist lînin XX,7.

$\S 93$. Steigerung der adjektiva.

Regelmässige steigerung. Komparativ: hêra IV,12; suiđra XVIII, 10; giungra XXI, 18 . Unregelmässige steigerung: betre IV, 52; mare V, 36; VIII, 53; XIX, 11; mara Prf. 7, 2; I, 50; $\mathrm{IV}, 12 ; \mathrm{X}, 29 ; \mathrm{XIV}, 12 ; \mathrm{XV}, 13$; arro $\mathrm{XV}, 18$; uyrse V, 14; maasta V, 20; maasto XV, 13; unresta, uirresta II, 10 rd.; cerist I, 41; VI, 62 ; foruost XVIII, 12; latmeste XVI, 23; hloetineste VI, 40; hloetmesta VI, 39; hloetmoeste Prf. 4, 19.

$\S 94$. Adverbia und ihre steigerung.

1) Adverbia auf -e sind: eaðe VIII, 19; hrceðe XI, 29; suiðe Prf. 7, 11; IV, 41; hucむre Prf. 4, 2; huocðre Prf. 2, 4; snude III,3; recone $\mathrm{V}, 9$; XI, 31 ; rccune $\mathrm{IV}, 27$; reccone $\mathrm{XVIII,} \mathrm{27;} \mathrm{symle}$ VI, 34; symble VII, 6; yfle III, 20; XVIII, 23; gere XXI, 12; giorne $\mathrm{XX}, 11$; suelce $\mathrm{I}, 14$; deigle $\mathrm{XIX}, 38$; aedgoeむre $\mathrm{XX}, 4$; cedgeadre XXI, 2. 13; daneben bildungen auf $-a$ : afra I, 18; V, 37; nofra XIX, 41; auf -a: afra VI, 35.

2) Adverbia auf -lice sind: soðlice Prf. 2, 3; cuðlice Prf. 3,11; monigfaldlice Prf. 7, 3; arlice VIII, 1; monigfallice Prf. 7,18; cirlice XXI, 4; boerlice XVI, 29; heartlice XII, 32; wundorlice IX, 30; gimonelice 188, 2; mit verlust des $e$ : geliclic Prf. 4, 10; бuslic VIII, 5.

3) Ws. giena entspricht geane IV, 35.

4) Adverbia auf -unga sind: longunga Prf. 7, 18; fullunga II, 6 rd.; suiugunga XI,28; anunga XXI, 25; daneben bildungen auf -ae, -e: eauunga XVIII, 20; eaunge VII, 4; XI, 14; euunge VII, 10; niunge III, 3; dernunge XVIII, 20; woenunge V, 46. 
DIE SPRACHE DER NORTHUMBR. INTERIJNEARVERSION. 97

5) Als adverbia werden gebraucht folgende oblique kasus von adjektiven und substantiven: soð Prf. 4, 18. 20; neh III,23; lytil XVI, 16; lyttil XVI, 17; lyttel XIII, 33; menigo IV, 41; lythuon Prf. 7, 14; togoegnes IV, 51; togoegnas XI, 20; ongregn XII, 18; tomiddes XX, 26; adune Prf. 2, 1; giosterdreg IV, 52; ođrae - uisa XIV, 11.

6) Adverbia auf ursprüngliches -an verlieren das auslautende $n$ : befora Prf. 3, 3; huona I, 48; II, 9; hiona II, 16; ufa Prf. 3, 7; binna XVIII,15; XX,26; heana VII, 3; бona Prf. 4, 11; soðða VI, 16; siðða 187, 14; buta Prf. 3,14 ; begeonda I, 28; begienda III, 26; bigeonda XIX, 18; der endvokal erscheint auch als $e$, a: huonne I, 48; VI, 25; begeande VI, 25; îte, bute XVIII, 16 ; bute XIX, 13; beforce XII, 37; $n$ ist bewahrt in neaðan VIII, 23 ; ûtan 188, 4 .

Beispiele von steigerung sind: komparativ: mce VII, 31; mara XII, 43; hraður XX, 4; forðor VIII, 11; XV, 2; suiðor III, 19; XIX, 8; suiður XXI, 15; wer Prf. 3, 3; 5, 18; 6, 11 etc.; doppelt gesteigert arrur I, 30; superlativ: cerist X, 40; XIX, 39 .

Die zusammenstellung der zahlwörter sowie der pronominalgattungen erübrigt sich durch Cook's "Glossary", das die belege vollständig für alle vier evangelien giebt.

\section{Litteratur.}

Bosworth-Toller, Anglo-Saxon Dictionary = B.-T.

Bülbring, K. D., Anglia, Beiblatt VII, IX, X.

Braune ${ }^{2}$, Wilh., Ahd. grammatik. Halle 1891.

Braune ${ }^{3}$, Wilh., Got. grammatik. Halle 1887.

Cosijn 2, P. J., Altwestsächische grammatik. Haag $1893=$ Cosijn.

Cook, A.S., A Glossary of the Old Northumbrian Gospels. Halle $1894=$ Cook.

Dieter, Laut- und formenlehre der altgermanischen dialekte I. Leipzig 1898. II. ebd. 1900.

Englische Studien ed. Hoops = E. St.

Fritzner, Ordbog over det gamle norske sprog. Kristiania 1886.

Grundriss der germanischen philologie $\mathrm{I}^{2}$ ed. Paul. Strassburg $1898=\mathrm{P}$. G. Hempl, Angl. XXII, 375-383.

Hilmer, Zur altnordhumbrischen laut- und flexionslehre. Goslarer programm $1882=$ Hilmer.

Kaluza, M., Historische grammatik der englischen sprache I. Berlin (Felber) $1900=$ Kaluza.

Anglia. N.F. XII. 
Kluge, F., Nominale stammbildungslehre der altgermanischen dialekte. ${ }^{2}$ Halle $1899=$ N. St. B.

Kluge-Lutz, English Etymology. Strassburg $1898=$ Kluge-Lutz.

Kluge, F., Etymologisches wörterbuch der deutschen sprache. ${ }^{\bullet}$ Strassburg 1899 = Kluge.

Lea, M. E., The language of the Northumbrian gloss to St. Mark. Anglia, $\mathrm{XVI}, 62-206=\mathrm{L}$.

Lindelöf, U., Die sprache des rituals von Durham etc. Helsingfors $1890=$ Lind.

Lindelöf, U., Beiträge zur kenntnis des altnorthumbrischen. Mémoires de la société neo-philologique à Helsingfors I (1893), 219-302.

Noreen, A., Altisländische und altnorwegische grammatik. Halle $1892=$ Noreen.

Paul, H., Zur geschichte des german. vokalismus. Beitr. VI.

Pogatscher, A., Zur lautlehre der griech., lat. und romanischen lehnwörter im altenglischen (Q.-F. 64). Strassburg $1888=$ Pog., Pogatscher.

Sievers, Angelsächsische grammatik. ${ }^{8}$ Halle $1898=\mathrm{S}$.

Streitberg, W., Urgermanische grammatik. Heidelberg 1896.

Sweet, H., History of English Sounds.' Oxford 1888.

Sweet, H., The Student's Anglo-Saxon Dictionary. Oxford 1897.

Zeuner, Die sprache des kentischen psalters. Halle $1881=$ Zeuner.

Whitney, Century Dictionary = C. D.

Anglia, Zeitschrift für englische philologie, ed. Einenkel \& Flügel.

Anglia, Beiblatt, ed. Mann.

Beiträge zur geschichte der deutschen sprache und literatur, ed. Sievers = Beitr.

Indogermanische forschungen. Zs. für idg. sprachen und altertumskunde. ed. Brugmann und Streitberg = I. F.

Bemerkung. Sievers, Zum ags. vokalismus, Leipziger dekanatsprogramm $1900 \mathrm{kam}$ mir leider erst zu gesicht, als der satz in allen teilen bereits abgeschlossen war.

Prf. bezieht sich anf ${ }_{n}$ Argumentum ${ }^{4}$ und "Capitula Lectionum" auf pp. 1-8 des textes und wird mit deutschen ziffern zitiert; pp.187. 188 enthalten ein nachwort der übersetzer.

\section{In h a l t.}

Teil I. Lautlehre. . . . . . . . . . . . . . . . . 3

Erster abschnitt: Vokalismus. . . . . . . . . . . . . 3

A) Die vokale der stammsilben. . . . . . . . . . . . 3

Kapitel I. Kurze vokale ( $\S 1-5)$. . . . . . . . . . 3

Kapitel II. Lange vokale (\$§ 6-10). . . . . . . . . 12 
IIE SPRACHE DER NORTHUMBR. INTERLINEARVERSION. 99

Kapitel III Diphthonge $(\$ \& 11-14)$ Seite

Kapitel IV. Beeinflussung betonter vokale durch nachbar-

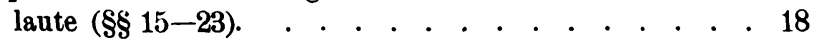

B) Die vokale der mittel- und endsilben. . . . . . . . . . 39

Kapitel V. Vokalwechsel $(\S \S 24,25)$. . . . . . . . . . 39

Kapitel VI. Vokalschwund $(\S \S 27,28)$. . . . . . . . . 43

Zweiter abschnitt: Konsonantismus. . . . . . . . . . . 45

Kapitel VII. Die halbvokale $(\S \S 29,30)$. . . . . . . . 45

Kapitel VIII. Die liquidae und nasale (§§ 31-34). . . . 48

Kapitel IX. Labiale ( $\S 35-37)$. . . . . . . . . . 50

Kapitel X. Dentale (\$§ $38-4 \mathrm{l})$. . . . . . . . . . . . . 51

Kapitel XI. Velare und palatale (\$§ 42-44). . . . . . . 53

Kapitel XII. Allgemeines über die konsonanten (\$§ 45-47). 56

Teil U. Flexionslehre. . . . . . . . . . . , . . . . 58

A) Verbalflexion. . . . . . . . . . . . . . . . . . . . . . . . 58

Kapitel XIII. Die endungen (\$\$ 48-64). . . . . . . . . 58

Kapitel XIV. Tempusbildung der starken verba ( $\$ 65-73) . \quad 67$

Kapitel XV. Tempusbildung der schwachen verba (\$§ 74-76). 76

Kapitel XVI. Kleinere gruppen (\$§77-81). . . . . . . 80

B) Nominalflexion. . . . . . . . . . . . . . . . . . . . . . . 83

Kapitel XVII. Deklination der substantiva ( $\S 81-90)$. . $\quad 83$

Kapitel XVIII. $\left\{\begin{array}{l}\text { Deklination der adjektiva (\$§ 91-94). } \\ \text { Adverbia. }\end{array}\right.$

Litteratur. . . . . . . . . . . . . . . . . . . . 97

Eisenberg. Hans Füchset. 